\title{
Process Algebra with Timing: Real Time and Discrete Time
}

\author{
J.C.M. Baeten ${ }^{1}$ and C.A. Middelburg ${ }^{1,2}$ \\ ${ }^{1}$ Computing Science Department, Eindhoven University of Technology \\ Eindhoven, the Netherlands \\ ${ }^{2}$ Department of Philosophy, Utrecht University \\ Utrecht, the Netherlands \\ $\{$ josb, keesm\}@win.tue.nl
}

\begin{abstract}
We present real time and discrete time versions of ACP with absolute timing and relative timing. The starting-point is a new real time version with absolute timing, called $\mathrm{ACP}^{\mathrm{sat}}$, featuring urgent actions and a delay operator. The discrete time versions are conservative extensions of the discrete time versions of $\mathrm{ACP}$ being known as $\mathrm{ACP}_{\text {dat }}$ and $\mathrm{ACP}_{\mathrm{drt}}$. The principal version is an extension of $\mathrm{ACP}^{\mathrm{sat}}$ with integration and initial abstraction to allow for choices over an interval of time and relative timing to be expressed. Its main virtue is that it generalizes ACP without timing and most other versions of ACP with timing in a smooth and natural way. This is shown for the real time version with relative timing and the discrete time version with absolute timing.

Keywords \& Phrases: process algebra, ACP, real time, discrete time, absolute timing, relative timing, two-phase scheme, time-stamping scheme.

1994 CR Categories: D.1.3, D.3.1, F.1.2, F.3.1.

Note: A slightly adapted version of this report will appear as a chapter in the forthcoming Handbook of Process Algebra (editors J.A. Bergstra, A. Ponse and S.A. Smolka) to be published by Elsevier.
\end{abstract}

\section{Introduction}

Algebraic concurrency theories such as ACP [13, 11, 10], CCS [33, 34] and CSP [18, 28] have been extended to deal with time-dependent behaviour in various ways. First of all, timing is either absolute or relative and the time scale on which time is measured is either continuous or discrete. Besides, execution of actions and passage of time are either separated or combined. Separation corresponds to the two-phase scheme of modeling time-dependent behaviour and combination corresponds to the time-stamping scheme.

Absolute timing and relative timing have been studied in the framework of ACP for both a continuous time scale and a discrete time scale. See e.g. [2] and [7]. The versions of $\mathrm{ACP}$ with timing where time is measured on a continuous time scale are 
usually called real time versions. In the remainder of this chapter, we adhere to this terminology. In the principal real time versions of $\mathrm{ACP}$, viz. $\mathrm{ACP} \rho$ and $\mathrm{ACPr} \rho$, which were both introduced in [2], and $\operatorname{ACP} \rho \sigma$, which was introduced in [4], execution of actions and passage of time are combined. On the contrary, they are separated in the principal discrete time versions of $\mathrm{ACP}$, viz. $\mathrm{ACP}_{\text {dat }}$ and $\mathrm{ACP}_{\text {drt }}$, which were both introduced in [7]. A real time version where execution of actions and passage of time are separated is ACPst, which was introduced in [6], and [8] focusses on discrete time versions where they are combined.

Measuring time on a discrete time scale does not mean that the execution of actions is restricted to discrete points in time. In the discrete time versions of ACP, time is divided into time slices and timing of actions is done with respect to the time slices in which they are performed - within a time slice there is only the order in which actions are performed. Thus, the discrete time versions permit to consider systems at a more abstract level than the real time case, a level where time is measured with finite precision. This also occurs in practice: software components of a system are executed on processors where the measure of time is provided by a discrete clock and, in case a physical system is controlled, the state of the physical system is sampled and adjusted at discrete points in time. In any case, the abstraction made in the discrete time versions makes the time-dependent behaviour of programs better amenable to analysis.

$\mathrm{ACP}$ can simply be embedded in the discrete time versions $\mathrm{ACP}_{\text {dat }}$ and $\mathrm{ACP}_{\mathrm{drt}}[7]$ by projecting the untimed process $a$ (for each action $a$ ) onto the delayable process $a-$ a delayable process $a$ is capable of performing the action $a$ in any time slice. Similarly, $\mathrm{ACP}$ can be embedded in the real time versions $\mathrm{ACP} \rho$ and $\operatorname{ACPr} \rho$ [2]. In other words, these discrete time and real time theories generalize the time free theory smoothly. Furthermore, in the discrete time case as well as the real time case, the relative time version can simply be embedded in the absolute time version extended with an initial abstraction operator to deal with relative timing.

However, the real time versions do not generalize the discrete time versions as smoothly as they generalize the time free theory. It turns out, as shown in [3], that the discrete time processes correspond to the real time processes for which the following holds: (1) if an action can be performed at some time $p \in \mathbb{R}$ such that $n<p<n+1$ $(n \in \mathbb{N})$, it can also be performed at any other time $p^{\prime} \in \mathbb{R}$ such that $n<p^{\prime}<n+1$; (2) no actions can be performed at times $p \in \mathbb{N}$. Clearly, such an embedding seriously lacks naturalness. The real time versions $\mathrm{ACP} \rho$ and $\operatorname{ACPr} \rho$ as well as the discrete time versions $\mathrm{ACP}_{\text {dat }}$ and $\mathrm{ACP}_{\text {drt }}$ are generalizations of $\mathrm{ACP}$ by intention. Since the real time versions were developed in advance of the discrete time versions, the former versions were not intentionally developed as generalizations of the latter versions. This explains at least partially the contrived embedding.

In this chapter, we present a new real time version of ACP with absolute timing which originates from $\operatorname{ACPs} \rho$, a real time version introduced in [6]. In this version, which features urgent actions and a delay operator, execution of actions and passage of time are separated. We explain how execution of actions and passage of time can be combined in this version. We further add an integration operator, with which a choice over an interval of time can be expressed, and an initial abstraction operator, with which relative timing can be expressed, to this version. We show how a real time version of ACP with relative timing, which originates from ACPst [6], can be embed- 
ded in the extended real time version with absolute timing. We also present discrete time versions of ACP with absolute timing and relative timing which are conservative extensions of $\mathrm{ACP}_{\text {dat }}$ and $\mathrm{ACP}_{\text {drt }}[7]$. We add an initial abstraction operator to the discrete time version with absolute timing as well. Showing how the discrete time version with relative timing can be embedded in the extended discrete time version with absolute timing, can be done similarly to the real time case. We show that the extended real time version generalizes the extended discrete time version smoothly. In this case, the following holds for those real time processes that correspond to the discrete time processes: if an action can be performed at some time $p \in \mathbb{R}$ such that $n \leq p<n+1(n \in \mathbb{N})$, it can also be performed at any other time $p^{\prime} \in \mathbb{R}$ such that $n \leq p^{\prime}<n+1$.

The main virtue of the extended real time version of ACP presented here is that it generalizes time free ACP as well as most other versions of ACP with timing in a smooth and natural way. The lack of a real time version of ACP with these characteristics was our main motivation to develop it. Different from the real time versions of [2] and [4], this version does not exclude the possibility of two or more actions to be performed consecutively at the same point in time. That is, it includes urgent actions, similar to ATP [37] and the different versions of CCS with timing [19, 35, 44]. This is useful in practice when describing and analyzing systems in which actions occur that are entirely independent. This is, for example, the case for actions that happen at different locations in a distributed system. In [2] and [4], the main idea was that it is difficult to imagine that actions are performed consecutively at the same point in time. But yet, this way of representing things is perfectly in line with modeling parallelism by interleaving. In point of fact it allows for independent actions to be handled faithfully.

In [2] and [4], ways to deal with independent actions are proposed where such actions take place at the same point in time by treating it as a special case of communication. This is, however, a real burden in the description and the analysis of the systems concerned. Of course, this does not limit the practical usefulness of ACP $\rho$ and $\operatorname{ACPr} \rho$ for systems in which no independent actions occur. The real time versions ACPs $\rho$ and ACPst of [6] simply do not exclude the possibility of two or more actions to be performed consecutively at the same point in time. Embedding in ACP $\rho$ and $\operatorname{ACPr} \rho$, respectively, is obtained by extending the time domain to a domain that includes non-standard real numbers. We conjecture that the real time version presented in this chapter, which originates from $\operatorname{ACPs} \rho$, can be embedded in $\mathrm{ACP} \rho$ as well.

We do not intend to give in this chapter a comprehensive overview of existing algebraic concurrency theories that deal with time-dependent behaviour. As suggested by the above, our aim is instead to present a coherent collection of algebraic concurrency theories that deal with time-dependent behaviour in different ways.

The structure of this chapter is as follows. First of all, in Section 2, we present the new real time version of ACP with absolute timing. We also explain how execution of actions and passage of time can be combined in this version. Then, in Section 3, we add integration and initial abstraction to this real time version of ACP. Next, in Section 4, we first present a real time version of ACP with relative timing and then show that it can be embedded in the real time version of ACP with absolute timing presented in Sections 2 and 3. After that, in Section 5, we first present conservative extensions of the discrete time versions $\mathrm{ACP}_{\text {dat }}$ and $\mathrm{ACP}_{\mathrm{drt}}$ of [7] and then show that 
the presented discrete time version with absolute timing can be embedded in the real time version with absolute timing presented in Sections 2 and 3. Finally, in Section 6, we make some concluding remarks.

\section{Real time process algebra: absolute timing}

In this section, we give the signature, axioms and term model of $\mathrm{ACP}^{\mathrm{sat}}$, a standard real time process algebra with absolute timing. In this theory, the non-negative standard real numbers $\left(\mathbb{R}_{\geq 0}\right)$ are used as the time domain. ACPsat originates from the theory $\operatorname{ACPs} \rho$, presented in [6]. Unlike $\operatorname{ACPs} \rho$, it separates execution of actions and passage of time.

In case of $\mathrm{ACP}^{\mathrm{sat}}$, it is assumed that a theory of the non-negative real numbers has been given. Its signature has to include the constant $0: \rightarrow \mathbb{R}_{\geq 0}$, the operator $+: \mathbb{R}_{\geq 0} \times \mathbb{R}_{\geq 0} \rightarrow \mathbb{R}_{\geq 0}$, and the predicates $\leq: \mathbb{R}_{\geq 0} \times \mathbb{R}_{\geq 0}$ and $=: \mathbb{R}_{\geq 0} \times \mathbb{R}_{\geq 0}$. In addition, this theory has to include axioms that characterize + as a commutative and associative operation with 0 as a neutral element and $\leq$ as a total ordering that has 0 as its least element and that is preserved by + .

In $\mathrm{ACP}^{\text {sat }}$, as in the other versions of $\mathrm{ACP}$ with timing presented in this chapter, it is assumed that a fixed but arbitrary set $\mathrm{A}$ of actions has been given. It is also assumed that a fixed but arbitrary communication function, i.e. a partial commutative and associative function $\gamma: \mathbf{A} \times \mathbf{A} \rightarrow \mathrm{A}$, has been given. The function $\gamma$ is regarded to give the result of the synchronous execution of any two actions for which this is possible, and to be undefined otherwise. The weak restrictions on $\gamma$ allow many kinds of communication between parallel processes to be modeled.

First, in Section 2.1, we treat $\mathrm{BPA}^{\text {sat }}$, basic standard real time process algebra with absolute timing, in which parallelism and communication are not considered. After that, in Section 2.2, $\mathrm{BPA}^{\text {sat }}$ is extended to $\mathrm{ACP}^{\mathrm{sat}}$ to deal with parallelism and communication as well. Finally, we demonstrate in Section 2.3 how one can combine execution of actions and passage of time in $\mathrm{ACP}^{\mathrm{sat}}$.

\subsection{Basic process algebra}

In $\mathrm{BPA}^{\text {sat }}$, we have the sort $\mathrm{P}$ of processes, the constants $\tilde{a}$ (one for each $a \in \mathrm{A}$ ), $\tilde{\delta}$ and $\dot{\delta}$, and the operators $\sigma_{\text {abs }}$ (absolute delay), $\cdot$ (sequential composition) and + (alternative composition). The constants $\tilde{a}$ stand for $a$ at time 0 . Similarly, the constant $\tilde{\delta}$ stands for a deadlock at time 0 . The constant $\dot{\delta}$ stands for an immediate deadlock, a process that exhibits inconsistent timing at time 0 . This means that $\dot{\delta}$, different from $\tilde{\delta}$, is not existing at time 0 . The process $\sigma_{\text {abs }}^{p}(x)$ is the process $x$ shifted in time by $p$. Thus, the process $\sigma_{\mathrm{abs}}^{p}(\tilde{a})$ is capable of first idling from time 0 to time $p$ and then upon reaching time $p$ performing action $a$, immediately followed by successful termination. The process $\sigma_{\text {abs }}^{p}(\tilde{\delta})$ is only capable of idling from time 0 to time $p$. Time $p$ can be reached by $\sigma_{\text {abs }}^{p}(\tilde{\delta})$. This is the difference with the process $\sigma_{\text {abs }}^{p}(\dot{\delta})$, which can only idle upto, but not including, time $p$. So $\sigma_{\text {abs }}^{p}(\dot{\delta})$ can not reach time $p$. The process $x \cdot y$ is the process $x$ followed upon successful termination by the process $y$. The process $x+y$ is the process that proceeds with either the process $x$ or the process $y$, but not both. As in the untimed case, the choice is resolved upon execution of the first action, and not before. We also have the auxiliary operators $v_{\text {abs }}$ 
(absolute time-out) and $\bar{v}_{\text {abs }}$ (absolute initialization). The process $v_{\text {abs }}^{p}(x)$ is the part of $x$ that starts to perform actions before time $p$. The process $\bar{v}_{\text {abs }}^{p}(x)$ is the part of $x$ that starts to perform actions at time $p$ or later.

A real time version of ACP with absolute timing where the notation $\tilde{a}$ was used earlier for urgent actions is $\operatorname{ACPs} \rho[6]$, but there it always carries a time-stamp. The binary operator $\sigma_{\text {abs }}$ generalizes the unary operator $\sigma_{\text {abs }}$ of $\mathrm{ACP}_{\text {dat }}$ [7] in a real time setting: for a real time process $x$ that corresponds to a discrete time process $x^{\prime}, \sigma_{\mathrm{abs}}^{1}(x)$ corresponds to $\sigma_{\text {abs }}\left(x^{\prime}\right)$. In earlier papers, including [2], [3], [4] and [6], the notations $x \gg p$ and $p \gg x$ were used instead of $v_{\mathrm{abs}}^{p}(x)$ and $\bar{v}_{\mathrm{abs}}^{p}(x)$, respectively. Besides, the time-out operator and the initialization operator were sometimes called the bounded initialization operator and the time shift operator, respectively.

It can be proved, using the axioms of $\mathrm{BPA}^{\text {sat }}$, that each process expressed using the auxiliary operators $v_{\text {abs }}$ and $\bar{v}_{\text {abs }}$ is equal to a process expressed without them. In other words, in $\mathrm{BPA}^{\text {sat}}$, all processes can be constructed from the constants using absolute delay, alternative composition and sequential composition only.

Signature of BPA ${ }^{\text {sat }}$ The signature of $\mathrm{BPA}^{\text {sat }}$ consists of the urgent action constants $\tilde{a}: \rightarrow \mathrm{P}$ (for each $a \in \mathrm{A}$ ), the urgent deadlock constant $\tilde{\delta}: \rightarrow \mathrm{P}$, the immediate deadlock constant $\dot{\delta}: \rightarrow \mathrm{P}$, the alternative composition operator $+: \mathrm{P} \times \mathrm{P} \rightarrow \mathrm{P}$, the sequential composition operator $: \mathrm{P} \times \mathrm{P} \rightarrow \mathrm{P}$, the absolute delay operator $\sigma_{\text {abs }}: \mathbb{R}_{\geq 0} \times \mathrm{P} \rightarrow \mathrm{P}$, the absolute time-out operator $v_{\text {abs }}: \mathbb{R}_{\geq 0} \times \mathrm{P} \rightarrow \mathrm{P}$, and the absolute initialization operator $\bar{v}_{\text {abs }}: \mathbb{R}_{\geq 0} \times \mathrm{P} \rightarrow \mathrm{P}$.

We assume that an infinite set of variables (of sort $\mathrm{P}$ ) has been given. Given the signature of $\mathrm{BPA}^{\text {sat }}$, terms of $\mathrm{BPA}^{\text {sat }}$, also referred to as process expressions, are constructed in the usual way. We will generally use infix notation for binary operators. The need to use parentheses is further reduced by ranking the precedence of the binary operators. Throughout this chapter we adhere to the following precedence rules: (i) the operator - has the highest precedence amongst the binary operators, (ii) the operator + has the lowest precedence amongst the binary operators, and (iii) all other binary operators have the same precedence. We will also use the following abbreviation. Let $\left(t_{i}\right)_{i \in \mathcal{I}}$ be an indexed set of terms of $\mathrm{BPA}^{\text {sat }}$ where $\mathcal{I}=\left\{i_{1}, \ldots, i_{n}\right\}$. Then we write $\sum_{i \in \mathcal{I}} t_{i}$ for $t_{i_{1}}+\ldots+t_{i_{n}}$. We further use the convention that $\sum_{i \in \mathcal{I}} t_{i}$ stands for $\dot{\delta}$ if $\mathcal{I}=\emptyset$.

We denote variables by $x, x^{\prime}, y, y^{\prime}, \ldots$. An important convention is that we use $a, a^{\prime}, b, b^{\prime}, \ldots$ to denote elements of $\mathrm{A} \cup\{\delta\}$ in the context of an equation, and elements of $\mathrm{A}$ in the context of an operational semantics rule. Furthermore, we use $H$ to denote a subset of $\mathrm{A}$. We denote elements of $\mathbb{R}_{\geq 0}$ by $p, p^{\prime}, q, q^{\prime}$ and elements of $\mathbb{R}_{>0}$ by $r, r^{\prime}$. We write $\mathrm{A}_{\delta}$ for $\mathrm{A} \cup\{\delta\}$.

Axioms of $\mathrm{BPA}^{\text {sat }}$ The axiom system of $\mathrm{BPA}^{\text {sat }}$ consists of the equations given in Tables 1 and 2 .

Axioms A1-A5 are common to ACP and all real and discrete time versions of ACP. Axioms A6ID and A7ID are simple reformulations of the axioms A6 and A7 of ACP: the constant $\delta$ has been replaced by the constant $\dot{\delta}$ - which is introduced because the intended interpretation of $\delta$ in $\mathrm{ACP}^{\text {sat }}$ differs from $\dot{\delta}$. These axioms or similar reformulations of A6 and A7 are found in all real and discrete time versions of ACP. 


\begin{tabular}{ll}
\hline$x+y=y+x$ & A1 \\
$(x+y)+z=x+(y+z)$ & A2 \\
$x+x=x$ & A3 \\
$(x+y) \cdot z=x \cdot z+y \cdot z$ & A4 \\
$(x \cdot y) \cdot z=x \cdot(y \cdot z)$ & A5 \\
$x+\dot{\delta}=x$ & A6ID \\
$\dot{\delta} \cdot x=\dot{\delta}$ & A7ID \\
\hline
\end{tabular}

Table 1: Axioms of BPA with immediate deadlock

\begin{tabular}{|c|c|}
\hline$\sigma_{\mathrm{abs}}^{0}(x)=\bar{v}_{\mathrm{abs}}^{0}(x)$ & SAT1 \\
\hline$\sigma_{\mathrm{abs}}^{p}\left(\sigma_{\mathrm{abs}}^{q}(x)\right)=\sigma_{\mathrm{abs}}^{p+q}(x)$ & SAT2 \\
\hline$\sigma_{\mathrm{abs}}^{p}(x)+\sigma_{\mathrm{abs}}^{p}(y)=\sigma_{\mathrm{abs}}^{p}(x+y)$ & SAT3 \\
\hline$\sigma_{\mathrm{abs}}^{p}(x) \cdot v_{\mathrm{abs}}^{p}(y)=\sigma_{\mathrm{abs}}^{p}(x \cdot \dot{\delta})$ & SAT4 \\
\hline$\sigma_{\mathrm{abs}}^{p}(x) \cdot\left(v_{\mathrm{abs}}^{p}(y)+\sigma_{\mathrm{abs}}^{p}(z)\right)=\sigma_{\mathrm{abs}}^{p}\left(x \cdot \bar{v}_{\mathrm{abs}}^{0}(z)\right)$ & SAT5 \\
\hline$\sigma_{\mathrm{abs}}^{p}(\dot{\delta}) \cdot x=\sigma_{\mathrm{abs}}^{p}(\dot{\delta})$ & SAT6 \\
\hline$\tilde{a}+\tilde{\delta}=\tilde{a}$ & A6SAa \\
\hline$\sigma_{\mathrm{abs}}^{r}(x)+\tilde{\delta}=\sigma_{\mathrm{abs}}^{r}(x)$ & $\mathrm{A} 6 \mathrm{SAb}$ \\
\hline$\tilde{\delta} \cdot x=\tilde{\delta}$ & A7SA \\
\hline$v_{\mathrm{abs}}^{p}(\dot{\delta})=\dot{\delta}$ & SATO0 \\
\hline$v_{\mathrm{abs}}^{0}(x)=\dot{\delta}$ & SATO1 \\
\hline$v_{\mathrm{abs}}^{r}(\tilde{a})=\tilde{a}$ & SATO2 \\
\hline$v_{\mathrm{abs}}^{p+q}\left(\sigma_{\mathrm{abs}}^{p}(x)\right)=\sigma_{\mathrm{abs}}^{p}\left(v_{\mathrm{abs}}^{q}(x)\right)$ & SATO3 \\
\hline$v_{\mathrm{abs}}^{p}(x+y)=v_{\mathrm{abs}}^{p}(x)+v_{\mathrm{abs}}^{p}(y)$ & SATO4 \\
\hline$v_{\mathrm{abs}}^{p}(x \cdot y)=v_{\mathrm{abs}}^{p}(x) \cdot y$ & SATO5 \\
\hline $\bar{v}_{\mathrm{abs}}^{0}(\dot{\delta})=\dot{\delta}$ & SAI0a \\
\hline $\bar{v}_{\mathrm{abs}}^{r}(\dot{\delta})=\sigma_{\mathrm{abs}}^{r}(\dot{\delta})$ & SAI0b \\
\hline $\bar{v}_{\mathrm{abs}}^{0}(\tilde{a})=\tilde{a}$ & SAI1 \\
\hline $\bar{v}_{\mathrm{abs}}^{r}(\tilde{a})=\sigma_{\mathrm{abs}}^{r}(\dot{\delta})$ & SAI2 \\
\hline $\bar{v}_{\mathrm{abs}}^{p+q}\left(\sigma_{\mathrm{abs}}^{p}(x)\right)=\sigma_{\mathrm{abs}}^{p}\left(\bar{v}_{\mathrm{abs}}^{q}\left(\bar{v}_{\mathrm{abs}}^{0}(x)\right)\right)$ & SAI3 \\
\hline $\bar{v}_{\mathrm{abs}}^{p}(x+y)=\bar{v}_{\mathrm{abs}}^{p}(x)+\bar{v}_{\mathrm{abs}}^{p}(y)$ & SAI4 \\
\hline $\bar{v}_{\mathrm{abs}}^{p}(x \cdot y)=\bar{v}_{\mathrm{abs}}^{p}(x) \cdot y$ & SAI5 \\
\hline
\end{tabular}

Table 2: Additional axioms for $\mathrm{BPA}^{\text {sat }}\left(a \in \mathrm{A}_{\delta}, p, q \geq 0, r>0\right)$

Axiom SAT1, and a few axioms treated later, become easier to understand by realizing that in $\mathrm{BPA}^{\text {sat }}$, as well as in $\mathrm{ACP}^{\text {sat }}$, the equation $\bar{v}_{\mathrm{abs}}^{0}(t)=t$ is derivable for all closed terms $t$. This equation expresses that initialization at time 0 has no effect on processes with absolute timing. To accommodate for the extension with initial abstraction in Section 3.2, we have used $\bar{v}_{\text {abs }}^{0}(x)$ instead of $x$ where the former is needed in the extension. Axioms SAT1 and SAT2 point out that a time shift by 0 has no effect in case of absolute timing and that consecutive time shifts add up. Axioms SAT3-SAT5 represent the interaction of absolute delay with alternative composition and sequential composition. Axiom SAT3, called the time factorization axiom, shows that passage of time by itself can not determine a choice. Axioms SAT4 and SAT5 express that if a process terminates successfully at some point in time, it can only be followed by the part of another process that starts to perform actions at the same time or 
later. Axiom SAT6 is a generalization of axiom A7ID. Using axioms A6SAa and $\mathrm{A} 6 \mathrm{SAb}$, the equation $t+\tilde{\delta}=t$ can be derived for all closed terms $t$ unless $t=\dot{\delta}$ - obviously $\dot{\delta}+\tilde{\delta}=\tilde{\delta}$. Axiom A7SA is another simple reformulation of axiom A7 of ACP. Axioms SATO0-SATO5 and SAI0-SAI5 reflect the intended meaning of the time-out and initialization operators clearly. Axioms SATO1 and SAI2 make precise what happens if a part that starts to perform actions before the time-out time and a part that starts to perform actions at the initialization time or later, respectively, do not exist. Equations SATO3' and SAI3' given in Table 3 are derivable from the axioms of $\mathrm{BPA}^{\text {sat }}$. In $\mathrm{BPA}^{\text {sat }}$ and $\mathrm{ACP}^{\text {sat }}$, and also in the further extension with

\begin{tabular}{ll}
\hline$v_{\mathrm{abs}}^{p}\left(\sigma_{\mathrm{abs}}^{p+q}(x)\right)=\sigma_{\mathrm{abs}}^{p}(\dot{\delta})$ & $\mathrm{SATO}^{\prime}$ \\
$\bar{v}_{\mathrm{abs}}^{p}\left(\sigma_{\mathrm{abs}}^{p+q}(x)\right)=\sigma_{\mathrm{abs}}^{p+q}(x)$ & $\mathrm{SAI}^{\prime}$ \\
$\bar{v}_{\mathrm{abs}}^{0}(x)=x$ & $\mathrm{SAI}^{\prime \prime}$ \\
$\bar{v}_{\mathrm{abs}}^{p+q}\left(\sigma_{\mathrm{abs}}^{p}(x)\right)=\sigma_{\mathrm{abs}}^{p}\left(\bar{v}_{\mathrm{abs}}^{q}(x)\right)$ & $\mathrm{SAI}^{\prime \prime}$ \\
\hline
\end{tabular}

Table 3: Some derivable equations and alternative axioms $(p, q \geq 0)$

initial abstraction, axiom SATO1 can be replaced by equation SATO3' just as well. In $\mathrm{BPA}^{\text {sat }}$ and $\mathrm{ACP}^{\mathrm{sat}}$, but not in the further extension with initial abstraction, axioms SAI0a, SAI1 and SAI3 together can be replaced by the equations SAI1" and SAI3" given in Table 3 . The absolute initialization operator could have been added later with the addition of the initial abstraction operator. However, having it available in $\mathrm{BPA}^{\text {sat }}$ and $\mathrm{ACP}^{\text {sat }}$ makes it possible to express interesting properties of real time processes with absolute timing such as the properties presented in Lemmas 1 and 3 below.

We can prove that the auxiliary operators $v_{\text {abs }}$ and $\bar{v}_{\text {abs }}$ can be eliminated in closed terms of BPA ${ }^{\text {sat }}$. We can also prove that sequential compositions in which the form of the first operand is not $\tilde{a}(a \in \mathrm{A})$ and alternative compositions in which the form of the first operand is $\sigma_{\text {abs }}^{p}(t)$ can be eliminated in closed terms of $\mathrm{BPA}^{\text {sat }}$. The terms that remain after exhaustive elimination are called the basic terms over BPA ${ }^{\text {sat }}$. Because of this elimination result, we are permitted to use induction on the structure of basic terms over $\mathrm{BPA}^{\text {sat }}$ to prove statements for all closed terms of $\mathrm{BPA}^{\text {sat }}$.

Examples We give some examples of a closed term of $\mathrm{BPA}^{\text {sat }}$ and the corresponding basic term:

$$
\begin{aligned}
& \sigma_{\mathrm{abs}}^{5}(\tilde{a}) \cdot \sigma_{\mathrm{abs}}^{4.9}(\tilde{b})=\sigma_{\mathrm{abs}}^{5}(\tilde{a} \cdot \dot{\delta}) \\
& \sigma_{\mathrm{abs}}^{5}(\tilde{a}) \cdot\left(\sigma_{\mathrm{abs}}^{4.9}(\tilde{b})+\sigma_{\mathrm{abs}}^{5.1}(\tilde{c})\right)=\sigma_{\mathrm{abs}}^{5}\left(\tilde{a} \cdot \sigma_{\mathrm{abs}}^{0.1}(\tilde{c})\right) \\
& v_{\mathrm{abs}}^{5}\left(\sigma_{\mathrm{abs}}^{4.9}(\tilde{a})+\sigma_{\mathrm{abs}}^{5.1}(\tilde{b})\right)=\sigma_{\mathrm{abs}}^{4.9}\left(\tilde{a}+\sigma_{\mathrm{abs}}^{0.1}(\dot{\delta})\right) \\
& \bar{v}_{\mathrm{abs}}^{5}\left(\sigma_{\mathrm{abs}}^{4.9}(\tilde{a})+\sigma_{\mathrm{abs}}^{5.1}(\tilde{b})\right)=\sigma_{\mathrm{abs}}^{5.1}(\tilde{b})
\end{aligned}
$$

The following lemmas are also useful in proofs. They are, for example, used in the proof of Theorem 12 (embedding of $\mathrm{ACP}^{\text {dat }} \sqrt{ }$ in $\mathrm{ACP}^{\mathrm{sat}} \mathrm{I} \sqrt{ }$ ). These lemmas, as most other lemmas in this chapter, call for proofs by induction on the structure of basic terms. The proofs are generally straightforward, but long and tedious. For that reason, we will present for each such proof only one of the cases to be treated. The selected case is usually typical of the proof and relatively hard. We write $\stackrel{\text { IH }}{=}$ to indicate that the induction hypothesis of the proof is used. 
Lemma 1 In $\mathrm{BPA}^{\text {sat }}$ and $\mathrm{ACP}^{\text {sat }}$, as well as in the further extensions with restricted integration and initial abstraction introduced in Section 3:

1. the equation $t=v_{\text {abs }}^{p}(t)+\bar{v}_{\text {abs }}^{p}(t)$ is derivable for all closed terms $t$ such that $t=\bar{v}_{\mathrm{abs}}^{0}(t)$ and $t=t+\sigma_{\mathrm{abs}}^{p}(\dot{\delta})$;

2. the equations $t=v_{\text {abs }}^{p}(t)$ and $\bar{v}_{\mathrm{abs}}^{p}(t)=\sigma_{\text {abs }}^{p}(\dot{\delta})$ are derivable for all closed terms $t$ such that $t=\bar{v}_{\mathrm{abs}}^{0}(t)$ and $t \neq t+\sigma_{\mathrm{abs}}^{p}(\dot{\delta})$.

Proof. It is straightforward to prove both 1 and 2 by induction on the structure of $t$.

1. We present only the case that $t$ is of the form $\sigma_{\text {abs }}^{q}\left(t^{\prime}\right)$. The other cases are similar, but simpler, and do not require case distinction.

$$
\begin{aligned}
& \text { Case } p \leq q: \sigma_{\mathrm{abs}}^{q}\left(t^{\prime}\right)+\sigma_{\mathrm{abs}}^{p}(\dot{\delta}) \stackrel{\mathrm{A} 1}{=} \sigma_{\mathrm{abs}}^{p}(\dot{\delta})+\sigma_{\mathrm{abs}}^{q}\left(t^{\prime}\right) \stackrel{\mathrm{SATO}^{\prime}, \mathrm{SAI}^{\prime}}{=} v_{\mathrm{abs}}^{p}\left(\sigma_{\mathrm{abs}}^{q}\left(t^{\prime}\right)\right)+\bar{v}_{\mathrm{abs}}^{p}\left(\sigma_{\mathrm{abs}}^{q}\left(t^{\prime}\right)\right) \\
& \text { Case } p>q: \sigma_{\text {abs }}^{q}\left(t^{\prime}\right)+\sigma_{\text {abs }}^{p}(\dot{\delta}) \stackrel{\text { SAT2 }}{=} \sigma_{\text {abs }}^{q}\left(t^{\prime}\right)+\sigma_{\text {abs }}^{q}\left(\sigma_{\text {abs }}^{p-q}(\dot{\delta})\right) \stackrel{\text { SAT3 }}{=} \sigma_{\text {abs }}^{q}\left(t^{\prime}+\sigma_{\text {abs }}^{p-q}(\dot{\delta})\right) \stackrel{\text { IH }}{=} \\
& \sigma_{\text {abs }}^{q}\left(v_{\text {abs }}^{p-q}\left(t^{\prime}\right)+\bar{v}_{\text {abs }}^{p-q}\left(t^{\prime}\right)\right) \stackrel{\text { SAT3 } 3}{=} \sigma_{\text {abs }}^{q}\left(v_{\text {abs }}^{p-q}\left(t^{\prime}\right)\right)+\sigma_{\text {abs }}^{q}\left(\bar{v}_{\text {abs }}^{p-q}\left(t^{\prime}\right)\right) \stackrel{\text { SATO3, SAI3 }}{=} \\
& v_{\text {abs }}^{p}\left(\sigma_{\text {abs }}^{q}\left(t^{\prime}\right)\right)+\bar{v}_{\text {abs }}^{p}\left(\sigma_{\text {abs }}^{q}\left(t^{\prime}\right)\right)
\end{aligned}
$$

In applying SAI3 we assume that $t^{\prime}=\bar{v}_{\text {abs }}^{0}\left(t^{\prime}\right)$. In case of $\mathrm{BPA}^{\text {sat }}, \mathrm{ACP}^{\text {sat }}$ and $\mathrm{ACP}^{\text {sat }}$ with integration, this equation is derivable for all closed terms $t^{\prime}$. The assumption is also justified in case of extension with initial abstraction. In that case, we are permitted, because of elimination results presented in Section 3.2, to consider here only closed terms of the form $\sigma_{\text {abs }}^{q}\left(t^{\prime}\right)$ where no initial abstraction occurs in $t^{\prime}$.

2. Observe that $\bar{v}_{\text {abs }}^{p}(t)=\sigma_{\text {abs }}^{p}(\dot{\delta})$ follows immediately from $t=v_{\text {abs }}^{p}(t)$ by axiom SI3. So it suffices to prove only $t=v_{\text {abs }}^{p}(t)$. Again, we present only the case that $t$ is of the form $\sigma_{\text {abs }}^{q}\left(t^{\prime}\right)$.

Case $p \leq q: \sigma_{\text {abs }}^{q}\left(t^{\prime}\right) \stackrel{\text { SAT2 }}{=} \sigma_{\text {abs }}^{p}\left(\sigma_{\text {abs }}^{q-p}\left(t^{\prime}\right)\right) \stackrel{\text { A6ID }}{=} \sigma_{\text {abs }}^{p}\left(\sigma_{\text {abs }}^{q-p}\left(t^{\prime}\right)+\dot{\delta}\right) \stackrel{\text { SAT3 }}{=}$

$\sigma_{\text {abs }}^{p}\left(\sigma_{\text {abs }}^{q-p}\left(t^{\prime}\right)\right)+\sigma_{\text {abs }}^{p}(\dot{\delta}) \stackrel{\text { SAT } 2}{=} \sigma_{\text {abs }}^{q}\left(t^{\prime}\right)+\sigma_{\text {abs }}^{p}(\dot{\delta})$

So $\sigma_{\text {abs }}^{q}\left(t^{\prime}\right) \neq \sigma_{\text {abs }}^{q}\left(t^{\prime}\right)+\sigma_{\text {abs }}^{p}(\dot{\delta})$ does not hold in case $p \leq q$

Case $p>q: \sigma_{\text {abs }}^{q}\left(t^{\prime}\right) \neq \sigma_{\text {abs }}^{q}\left(t^{\prime}\right)+\sigma_{\text {abs }}^{p}(\dot{\delta}) \stackrel{\text { SAT2,SAT3 }}{\Rightarrow} t^{\prime} \neq t^{\prime}+\sigma_{\text {abs }}^{p-q}(\dot{\delta})$

By the induction hypothesis, $\sigma_{\text {abs }}^{q}\left(t^{\prime}\right)=\sigma_{\text {abs }}^{q}\left(v_{\text {abs }}^{p-q}\left(t^{\prime}\right)\right) \stackrel{\text { SATO3 }}{=} v_{\text {abs }}^{p}\left(\sigma_{\text {abs }}^{q}\left(t^{\prime}\right)\right)$

From Lemma 1 we readily conclude the following.

Corollary 2 In $\mathrm{BPA}^{\mathrm{sat}}$ and $\mathrm{ACP}^{\mathrm{sat}}$, as well as in the further extensions with restricted integration and initial abstraction introduced in Section 3, the equation $\sigma_{\mathrm{abs}}^{p}(t)$. $t^{\prime}=\sigma_{\mathrm{abs}}^{p}(t) \cdot \bar{v}_{\mathrm{abs}}^{p}\left(t^{\prime}\right)$ is derivable for all closed terms $t$ and $t^{\prime}$ such that $t^{\prime}=\bar{v}_{\mathrm{abs}}^{0}\left(t^{\prime}\right)$.

Lemma 3 In $\mathrm{BPA}^{\text {sat }}$ and $\mathrm{ACP}^{\text {sat }}$, as well as in the further extensions with restricted integration and initial abstraction introduced in Section 3, for each $p \in \mathbb{R}_{\geq 0}$ and each closed term $t$, there exists a closed term $t^{\prime}$ such that $\bar{v}_{\text {abs }}^{p}(t)=\sigma_{\text {abs }}^{p}\left(t^{\prime}\right)$ and $\bar{t}^{\prime}=\bar{v}_{\text {abs }}^{0}\left(t^{\prime}\right)$. In subsequent proofs, we write $t_{[p]}$ for a fixed but arbitrary closed term $t^{\prime}$ that fulfills these conditions.

Proof. It is straightforward to prove this by induction on the structure of $t$. We present only the case that $t$ is of the form $\sigma_{\mathrm{abs}}^{q}\left(t^{\prime \prime}\right)$. Again, the other cases are similar, but simpler, and do not require case distinction.

$$
\begin{aligned}
\text { Case } p \leq q: & \bar{v}_{\text {abs }}^{p}\left(\sigma_{\text {abs }}^{q}\left(t^{\prime \prime}\right)\right) \stackrel{\text { SAI3 } 3^{\prime}}{=} \sigma_{\text {abs }}^{q}\left(t^{\prime \prime}\right) \stackrel{\text { SAT2 }}{=} \sigma_{\text {abs }}^{p}\left(\sigma_{\text {abs }}^{q-p}\left(t^{\prime \prime}\right)\right) \text { and } \\
& \sigma_{\text {abs }}^{q-p}\left(t^{\prime \prime}\right) \stackrel{\text { SAT2 }}{=} \sigma_{\text {abs }}^{0}\left(\sigma_{\text {abs }}^{q-p}\left(t^{\prime \prime}\right)\right) \stackrel{\text { SAAT }}{=} \bar{v}_{\text {abs }}^{0}\left(\sigma_{\text {abs }}^{q-p}\left(t^{\prime \prime}\right)\right) \\
\text { Case } p>q: & \bar{v}_{\text {abs }}^{p}\left(\sigma_{\text {abs }}^{q}\left(t^{\prime \prime}\right)\right) \stackrel{\text { SAI3 }}{=} \sigma_{\text {abs }}^{q}\left(\bar{v}_{\text {abs }}^{p-q}\left(t^{\prime \prime}\right)\right) \stackrel{\text { IH }}{=} \sigma_{\text {abs }}^{q}\left(\sigma_{\text {abs }}^{p-q}\left(t_{[p-q]}^{\prime \prime}\right)\right) \stackrel{\text { SAT2 } 2}{=} \sigma_{\text {abs }}^{p}\left(t_{[p-q]}^{\prime \prime}\right) \text { and } \\
& t_{[p-q]}^{\prime \prime}=\bar{v}_{\text {abs }}^{0}\left(t_{[p-q]}^{\prime \prime}\right)
\end{aligned}
$$


In applying SAI3 we assume that $t^{\prime \prime}=\bar{v}_{\text {abs }}^{0}\left(t^{\prime \prime}\right)$. As described in the previous proof, this assumption is justified in all cases.

Lemma 1 indicates that a process that is able to reach time $p$ can be regarded as being the alternative composition of the part that starts to perform actions before $p$ and the part that starts to perform actions at $p$ or later. Lemma 3 shows that the part of a process that starts to perform actions at time $p$ or later can always be regarded as a process shifted in time by $p$.

Semantics of $\mathrm{BPA}^{\text {sat }}$ A real time transition system over A consists of a set of states $\mathrm{S}$, a root state $\rho \in \mathrm{S}$ and four kinds of relations on states:

a binary relation $\langle-, p\rangle \stackrel{a}{\longrightarrow}\langle-, p\rangle$ for each $a \in \mathrm{A}, p \in \mathbb{R}_{\geq 0}$, a unary relation $\left\langle \_, p\right\rangle \stackrel{a}{\rightarrow}\langle\sqrt{ }, p\rangle$ for each $a \in \mathrm{A}, p \in \mathbb{R}_{\geq 0}$, a binary relation $\left\langle_{-}, p\right\rangle \stackrel{r}{\longmapsto}\left\langle\left\langle_{-}, q\right\rangle\right.$ for each $r \in \mathbb{R}_{>0}, p, q \in \mathbb{R}_{\geq 0}$ where $q=p+r$, a unary relation $\operatorname{ID}(-, p) \quad$ for each $p \in \mathbb{R}_{\geq 0}$;

satisfying

1. if $\langle s, p\rangle \stackrel{r+r^{\prime}}{\longmapsto}\left\langle s^{\prime}, q\right\rangle, r, r^{\prime}>0$, then there is a $s^{\prime \prime}$ such that $\langle s, p\rangle \stackrel{r}{\longmapsto}\left\langle s^{\prime \prime}, p+r\right\rangle$ and $\left\langle s^{\prime \prime}, p+r\right\rangle \stackrel{r^{\prime}}{\longmapsto}\left\langle s^{\prime}, q\right\rangle$;

2. if $\langle s, p\rangle \stackrel{r}{\longmapsto}\left\langle s^{\prime \prime}, p+r\right\rangle$ and $\left\langle s^{\prime \prime}, p+r\right\rangle \stackrel{r^{\prime}}{\longmapsto}\left\langle s^{\prime}, q\right\rangle$, then $\langle s, p\rangle \stackrel{r+r^{\prime}}{\longmapsto}\left\langle s^{\prime}, q\right\rangle$.

The four kinds of relations are called action step, action termination, time step and immediate deadlock relations, respectively. We write RTTS(A) for the set of all real time transition systems over $A$.

We shall associate a transition system TS $(t)$ in RTTS(A) with a closed term $t$ of $\mathrm{BPA}^{\text {sat }}$ by taking the set of closed terms of $\mathrm{BPA}^{\text {sat }}$ as set of states, the closed term $t$ as root state, and the action step, action termination, time step and immediate deadlock relations defined below using rules in the style of Plotkin [38]. A semantics given in this way is called a structural operational semantics. On the basis of these rules, the operators of $\mathrm{BPA}^{\text {sat }}$ can also be directly defined on the set of real time transition systems in a straightforward way. Note that, by taking closed terms as states, the relations can be explained as follows:

$$
\begin{aligned}
& \langle t, p\rangle \stackrel{a}{\longrightarrow}\left\langle t^{\prime}, p\right\rangle \text { : process } t \text { is capable of first performing action } a \text { at time } p \\
& \text { and then proceeding as process } t^{\prime} \text {; } \\
& \langle t, p\rangle \stackrel{a}{\longrightarrow}\langle\sqrt{ }, p\rangle \text { : process } t \text { is capable of first performing action } a \text { at time } p \\
& \text { and then terminating successfully; } \\
& \langle t, p\rangle \stackrel{r}{\longmapsto}\left\langle t^{\prime}, q\right\rangle \text { : process } t \text { is capable of first idling from time } p \text { to time } q \\
& \text { and then proceeding as process } t^{\prime} \text {; } \\
& \mathrm{ID}(t, p): \quad \text { process } t \text { is not capable of reaching time } p \text {. }
\end{aligned}
$$

The rules for the operational semantics have the form $\frac{p_{1}, \ldots, p_{m}, s}{c_{1}, \ldots, c_{n}}$, where $s$ is optional. They are to be read as "if $p_{1}$ and $\ldots$ and $p_{m}$ then $c_{1}$ and $\ldots$ and $c_{n}$, provided $s "$. As customary, $p_{1}, \ldots, p_{m}$ and $c_{1}, \ldots, c_{n}$ are called the premises and the conclusions, respectively. The conclusions of a rule are positive formulas of the form $\langle t, p\rangle \stackrel{a}{\longrightarrow}\left\langle t^{\prime}, p\right\rangle,\langle t, p\rangle \stackrel{a}{\longrightarrow}\langle\sqrt{ }, p\rangle,\langle t, p\rangle \stackrel{r}{\longrightarrow}\left\langle t^{\prime}, q\right\rangle$ or $\operatorname{ID}(t, p)$, where $t$ and $t^{\prime}$ are open 
terms of $\mathrm{BPA}^{\text {sat }}$. The premises of a rule are positive formulas of the above forms or negative formulas of the form $\neg \mathrm{ID}(t, p)$. The rules are actually rule schemas. The optional $s$ is a side-condition restricting the actions over which $a, b$ and $c$ range and the non-negative real numbers over which $p, q$ and $r$ range. Within the framework of term deduction systems introduced in [9], the instances of the rule schemas that satisfy the stated side-conditions should be taken as the rules under consideration. For the rest, we continue to use the word rule in the broader sense.

The signature of $\mathrm{BPA}^{\text {sat }}$ together with the rules that will be given constitute according to the definitions of [43] a strictly stratifiable term deduction system. For a term deduction system having rules with negative premises, it is not immediately clear whether the term deduction system is meaningful. That is, it is not clear whether there exist relations for which exactly those formulas hold that can be derived using the rules of the term deduction system (see e.g. [25] and [15]). However, if the term deduction system is stratifiable then there exist such relations. If it is strictly stratifiable then there exist unique such relations. The restriction to stratifiable term deduction systems is essential for the congruence theorem mentioned below.

Term deduction systems support only unary and binary relations on closed terms over some one-sorted signature. Generalization to the many-sorted case is harmless if it is confined to unary and binary relations on closed terms of one of the sorts. Therefore, we chose to have, for instance, many binary action step relations for each action in $A$, viz. one for each element of $\mathbb{R}_{\geq 0}$, instead of one ternary or quaternary relation.

For the operational semantics of $\mathrm{BPA}^{\text {sat }}$, as well as $\mathrm{ACP}^{\mathrm{sat}}$ and the further extensions described in Section 3, we will only define time step relations for which $\langle t, p\rangle \stackrel{r}{\longmapsto}\left\langle t^{\prime}, q\right\rangle$ holds only if $t \equiv t^{\prime}$. The given rules define relations for which exactly those formulas hold that can be derived using the rules. Consequently, there are no rules with conclusions of the form $\langle x, p\rangle \stackrel{r}{\longmapsto}\left\langle x^{\prime}, q\right\rangle$ where $x \not \equiv x^{\prime}$. Hence, it makes no difference if in a rule a premise of the form $\langle x, p\rangle \stackrel{r}{\longmapsto}\langle x, q\rangle$ is replaced by $\langle x, p\rangle \stackrel{r}{\longmapsto}\left\langle x^{\prime}, q\right\rangle$ if $x^{\prime}$ is a variable different from the variables occurring in the rule. We prefer the premises of the form $\langle x, p\rangle \stackrel{r}{\longmapsto}\langle x, q\rangle$ because they are better suited to a natural explanation of the rules. However, the replacements are usually needed whenever general results about term deduction systems, e.g. results of [43], are used. In the remainder of this chapter, we will refrain from making mention of the need for the replacements because they are trivial and make no difference with respect to the relations defined.

The structural operational semantics of $\mathrm{BPA}^{\text {sat }}$ is described by the rules given in Table 4.

These rules are easy to understand. We will only explain the rules for the absolute delay operator $\left(\sigma_{\mathrm{abs}}\right)$. The first pair of rules expresses that the action related capabilities of a process $\sigma_{\text {abs }}^{0}(x)$ at time $p$ include those of process $x$ at time $p$. The second pair of rules expresses that the action related capabilities of a process $\sigma_{\text {abs }}^{r}(x)$ at time $p+r$ include those of process $x$ at time $p$ shifted in time by $r(p \geq 0, r>0)$. The third pair of rules expresses that the time related capabilities of a process $\sigma_{\mathrm{abs}}^{q}(x)$ at time $p+q$ include those of process $x$ at time $p$ shifted in time by $q(q \geq 0)$. The fourth pair of rules expresses that a process $\sigma_{\mathrm{abs}}^{r}(x)$ can idle from any time $p \geq 0$ to any time $q<r$ and that it can also idle to time $r$ provided that process $x$ can reach time 0 . 


\begin{tabular}{|c|c|}
\hline $\operatorname{ID}(\dot{\delta}, p) \quad \operatorname{ID}(\tilde{\delta}, r)$ & $\langle\tilde{a}, 0\rangle \stackrel{a}{\longrightarrow}\langle\sqrt{ }, 0\rangle$ \\
\hline$\langle x, p\rangle \stackrel{a}{\longrightarrow}\left\langle x^{\prime}, p\right\rangle$ & $\langle x, p\rangle \stackrel{a}{\longrightarrow}\langle\sqrt{ }, p\rangle$ \\
\hline$\left\langle\sigma_{\mathrm{abs}}^{0}(x), p\right\rangle \stackrel{a}{\longrightarrow}\left\langle x^{\prime}, p\right\rangle$ & $\left\langle\sigma_{\mathrm{abs}}^{0}(x), p\right\rangle \stackrel{a}{\longrightarrow}\langle\sqrt{ }, p\rangle$ \\
\hline$\langle x, p\rangle \stackrel{a}{\longrightarrow}\left\langle x^{\prime}, p\right\rangle$ & $\langle x, p\rangle \stackrel{a}{\longrightarrow}\langle\sqrt{ }, p\rangle$ \\
\hline$\left\langle\sigma_{\mathrm{abs}}^{r}(x), p+r\right\rangle \stackrel{a}{\longrightarrow}\left\langle\sigma_{\mathrm{abs}}^{r}\left(x^{\prime}\right), p+r\right\rangle$ & $\left\langle\sigma_{\mathrm{abs}}^{r}(x), p+r\right\rangle \stackrel{a}{\longrightarrow}\langle\sqrt{ }, p+r\rangle$ \\
\hline$\langle x, p\rangle \stackrel{r}{\longmapsto}\langle x, p+r\rangle$ & $\mathrm{ID}(x, p)$ \\
\hline$\left\langle\sigma_{\mathrm{abs}}^{q}(x), p+q\right\rangle \stackrel{r}{\longmapsto}\left\langle\sigma_{\mathrm{abs}}^{q}(x), p+q+r\right\rangle$ & $\operatorname{ID}\left(\sigma_{\mathrm{abs}}^{q}(x), p+q\right)$ \\
\hline$q>p$ & $\neg \mid \mathrm{D}(x, 0)$ \\
\hline$\left\langle\sigma_{\mathrm{abs}}^{q+r}(x), p\right\rangle \stackrel{r}{\longmapsto}\left\langle\sigma_{\mathrm{abs}}^{q+r}(x), p+r\right\rangle$ & $\left\langle\sigma_{\mathrm{abs}}^{q+r}(x), q\right\rangle \stackrel{r}{\longmapsto}\left\langle\sigma_{\mathrm{abs}}^{q+r}(x), q+r\right\rangle$ \\
\hline$\langle x, p\rangle \stackrel{a}{\longrightarrow}\left\langle x^{\prime}, p\right\rangle$ & $\langle x, p\rangle \stackrel{a}{\longrightarrow}\langle\sqrt{ }, p\rangle$ \\
\hline $\begin{array}{l}\langle x+y, p\rangle \stackrel{a}{\longrightarrow}\left\langle x^{\prime}, p\right\rangle \\
\langle y+x, p\rangle \stackrel{a}{\longrightarrow}\left\langle x^{\prime}, p\right\rangle\end{array}$ & $\begin{array}{l}\langle x+y, p\rangle \stackrel{a}{\longrightarrow}\langle\sqrt{ }, p\rangle, \\
\langle y+x, p\rangle \stackrel{a}{\longrightarrow}\langle\sqrt{ }, p\rangle\end{array}$ \\
\hline$\langle x, p\rangle \stackrel{r}{\longmapsto}\langle x, p+r\rangle$ & $\operatorname{ID}(x, p), \operatorname{ID}(y, p)$ \\
\hline $\begin{array}{l}\langle x+y, p\rangle \stackrel{r}{\longmapsto}\langle x+y, p+r\rangle, \\
\langle y+x, p\rangle \stackrel{r}{\longmapsto}\langle y+x, p+r\rangle\end{array}$ & $\mathrm{ID}(x+y, p)$ \\
\hline$\langle x, p\rangle \stackrel{a}{\longrightarrow}\left\langle x^{\prime}, p\right\rangle$ & $\langle x, p\rangle \stackrel{a}{\longrightarrow}\langle\sqrt{ }, p\rangle$ \\
\hline$\langle x \cdot y, p\rangle \stackrel{a}{\longrightarrow}\left\langle x^{\prime} \cdot y, p\right\rangle$ & $\langle x \cdot y, p\rangle \stackrel{a}{\longrightarrow}\langle y, p\rangle$ \\
\hline$\langle x, p\rangle \stackrel{r}{\longmapsto}\langle x, p+r\rangle$ & $\mathrm{ID}(x, p)$ \\
\hline$\langle x \cdot y, p\rangle \stackrel{r}{\longmapsto}\langle x \cdot y, p+r\rangle$ & $\mathrm{ID}(x \cdot y, p)$ \\
\hline$\langle x, p\rangle \stackrel{a}{\longrightarrow}\left\langle x^{\prime}, p\right\rangle, q>p$ & $\langle x, p\rangle \stackrel{a}{\longrightarrow}\langle\sqrt{ }, p\rangle, q>p$ \\
\hline$\left\langle v_{\mathrm{abs}}^{q}(x), p\right\rangle \stackrel{a}{\longrightarrow}\left\langle x^{\prime}, p\right\rangle$ & $\left\langle v_{\mathrm{abs}}^{q}(x), p\right\rangle \stackrel{a}{\longrightarrow}\langle\sqrt{ }, p\rangle$ \\
\hline$\langle x, p\rangle \stackrel{r}{\longmapsto}\langle x, p+r\rangle, q>p+r$ & $\mathrm{ID}(x, p), q>p$ \\
\hline$\left\langle v_{\mathrm{abs}}^{q}(x), p\right\rangle \stackrel{r}{\longmapsto}\left\langle v_{\mathrm{abs}}^{q}(x), p+r\right\rangle$ & $\operatorname{ID}\left(v_{\mathrm{abs}}^{q}(x), p\right)$ \\
\hline$q \leq p$ & \\
\hline $\mathrm{ID}\left(v_{\mathrm{abs}}^{q}(x), p\right)$ & \\
\hline$\langle x, p\rangle \stackrel{a}{\longrightarrow}\left\langle x^{\prime}, p\right\rangle, q \leq p$ & $\langle x, p\rangle \stackrel{a}{\longrightarrow}\langle\sqrt{ }, p\rangle, q \leq p$ \\
\hline$\left\langle\bar{v}_{\mathrm{abs}}^{q}(x), p\right\rangle \stackrel{a}{\longrightarrow}\left\langle x^{\prime}, p\right\rangle$ & $\left\langle\bar{v}_{\mathrm{abs}}^{q}(x), p\right\rangle \stackrel{a}{\longrightarrow}\langle\sqrt{ }, p\rangle$ \\
\hline$\langle x, p\rangle \stackrel{r}{\longmapsto}\langle x, p+r\rangle, q \leq p+r$ & $\mathrm{ID}(x, p), q \leq p$ \\
\hline$\left\langle\bar{v}_{\mathrm{abs}}^{q}(x), p\right\rangle \stackrel{r}{\longmapsto}\left\langle\bar{v}_{\mathrm{abs}}^{q}(x), p+r\right\rangle$ & $\mathrm{ID}\left(\bar{v}_{\mathrm{abs}}^{q}(x), p\right)$ \\
\hline$q>p$ & $\neg \mathrm{ID}(x, q+r)$ \\
\hline$\left\langle\bar{v}_{\mathrm{abs}}^{q+r}(x), p\right\rangle \stackrel{r}{\longmapsto}\left\langle\bar{v}_{\mathrm{abs}}^{q+r}(x), p+r\right\rangle$ & $\left\langle\bar{v}_{\mathrm{abs}}^{q+r}(x), q\right\rangle \stackrel{r}{\longmapsto}\left\langle\bar{v}_{\mathrm{abs}}^{q+r}(x), q+r\right\rangle$ \\
\hline
\end{tabular}

Table 4: Rules for operational semantics of $\mathrm{BPA}^{\text {sat }}(a \in \mathrm{A}, r>0, p, q \geq 0)$

By identifying bisimilar processes we obtain our preferred model of BPA ${ }^{\text {sat }}$. One process is (strongly) bisimilar to another process means that if one of the processes is capable of doing a certain step, i.e. performing a certain action at a certain time or idling from a certain time to another, and next going on as a certain subsequent process then the other process is capable of doing the same step and next going on as a process bisimilar to the subsequent process. More precisely, a bisimulation on 
RTTS(A) is a symmetric binary relation $R$ on the set of states $S$ such that:

1. if $R(s, t)$ and $\langle s, p\rangle \stackrel{a}{\longrightarrow}\left\langle s^{\prime}, p\right\rangle$, then there is a $t^{\prime}$ such that $\langle t, p\rangle \stackrel{a}{\longrightarrow}\left\langle t^{\prime}, p\right\rangle$ and $R\left(s^{\prime}, t^{\prime}\right)$;

2. if $R(s, t)$, then $\langle s, p\rangle \stackrel{a}{\rightarrow}\langle\sqrt{ }, p\rangle$ iff $\langle t, p\rangle \stackrel{a}{\longrightarrow}\langle\sqrt{ }, p\rangle$;

3. if $R(s, t)$ and $\langle s, p\rangle \stackrel{r}{\longmapsto}\left\langle s^{\prime}, q\right\rangle$, then there is a $t^{\prime}$ such that $\langle t, p\rangle \stackrel{r}{\longmapsto}\left\langle t^{\prime}, q\right\rangle$ and $R\left(s^{\prime}, t^{\prime}\right)$;

4. if $R(s, t)$, then $\operatorname{ID}(s, p)$ iff $\operatorname{ID}(t, p)$.

We say that two closed terms $s$ and $t$ are bisimilar, written $s \overleftrightarrow{t}$, if there exists a bisimulation $R$ such that $R(s, t)$.

It is known from [43] that if a stratifiable term deduction system is in panth format, bisimulation equivalence as defined here is a congruence for the operators in the signature concerned. This collection of constraints on the form of the rules of a term deduction system is defined in [43] for the one-sorted case, but in case of the real time and discrete time versions of ACP presented in this chapter we have in addition to the sort of processes also the sort of non-negative real numbers or the sort of natural numbers. In order to conform to the panth format as defined in [43], the number of constants and operators in the first argument of a conclusion must be either zero or one. In case of most term deduction systems given in this chapter, this condition is only fulfilled if we disregard constants and operators that do not yield processes. Careful checking of the proof of the congruence theorem for the panth format given in [43], the only result about the panth format that we will use, shows that the result goes through for the many-sorted case if the above-mentioned condition is relaxed in such a way that only the number of constants and operators that yield processes is restricted to zero or one. Therefore, we will refer to this panth-like format in the remainder of this chapter as the panth format. We note here that checking of the proof of the congruence theorem includes checking of the proofs of many related lemmas and theorems given in [15] and [43]. For a comprehensive introduction to rule formats guaranteeing that bisimulation equivalence is a congruence, the reader is referred to [1].

The signature of $\mathrm{BPA}^{\text {sat }}$ together with the rules for the operational semantics of BPA $^{\text {sat }}$ constitute a stratifiable term deduction system in panth format. Consequently, bisimulation equivalence is a congruence for the operators of BPA ${ }^{\text {sat }}$. For this reason, the operators of $\mathrm{BPA}^{\text {sat }}$ can be defined on the set of bisimulation equivalence classes. We can prove that this results in a model for $\mathrm{BPA}^{\text {sat }}$, i.e. all equations derivable in $\mathrm{BPA}^{\text {sat }}$ hold. In other words, the axioms of BPA sat form a sound axiomatization for the model based on bisimulation equivalence classes. As in the case of the other axiomatizations presented in this chapter, we leave it as an open problem whether the axioms of $\mathrm{BPA}^{\text {sat }}$ form a complete axiomatization for this model.

\subsection{Algebra of communicating processes}

In $\mathrm{ACP}^{\text {sat }}$, we have, in addition to sequential and alternative composition, parallel composition of processes. The process $x \| y$ is the process that proceeds with the processes $x$ and $y$ in parallel. Furthermore, we have the encapsulation operators $\partial_{H}$ (one for each $H \subseteq \mathrm{A}$ ) which turns all urgent actions $\tilde{a}$, where $a \in H$, into $\tilde{\delta}$. As in ACP,

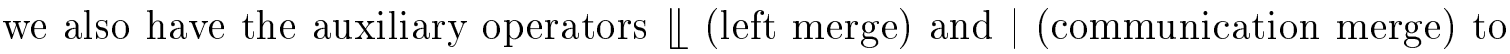


get a finite axiomatization of the parallel composition operator. The processes $x \llbracket y$ and $x \| y$ are the same except that $x \Perp y$ must start to perform actions by performing an action of $x$. The processes $x \mid y$ and $x \| y$ are the same except that $x \mid y$ must start to perform actions by performing an action of $x$ and an action of $y$ synchronously. In case of $\mathrm{ACP}^{\mathrm{sat}}$, an additional auxiliary operator $\nu_{\text {abs }}$ (absolute urgent initialization) is needed. The process $\nu_{\text {abs }}(x)$ is the part of process $x$ that starts to perform actions at time 0 .

The operator $\nu_{\mathrm{abs}}$ of $\mathrm{ACP}{ }^{\mathrm{sat}}$ is simply the operator $\nu_{\mathrm{abs}}$ of $\mathrm{ACP}_{\text {dat }}^{\tau}$ [5] lifted to the real time setting.

Signature of $\mathbf{A C P}^{\text {sat }}$ The signature of $\mathrm{ACP}^{\text {sat }}$ is the signature of $\mathrm{BPA}^{\text {sat }}$ extended with the parallel composition operator $\|: \mathrm{P} \times \mathrm{P} \rightarrow \mathrm{P}$, the left merge operator $\|$ : $\mathrm{P} \times \mathrm{P} \rightarrow \mathrm{P}$, the communication merge operator $\mid: \mathrm{P} \times \mathrm{P} \rightarrow \mathrm{P}$, the encapsulation operators $\partial_{H}: \mathrm{P} \rightarrow \mathrm{P}$ (for each $H \subseteq \mathrm{A}$ ), and the absolute urgent initialization operator $\nu_{\text {abs }}: \mathrm{P} \rightarrow \mathrm{P}$.

Axioms of $\mathrm{ACP}^{\text {sat }}$ The axiom system of $\mathrm{ACP}^{\text {sat }}$ consists of the axioms of $\mathrm{BPA}^{\text {sat }}$ and the equations given in Table 5 .

Axioms CM1, CM4, CM8, CM9, D3 and D4 are common to ACP and all real and discrete time versions of ACP. Axioms CF1SA, CF2SA, CM2SA, CM3SA, CM5SACM7SA, D1SA and D2SA are simple reformulations of the axioms CF1, CF2, CM2, CM3, CM5-CM7, D1 and D2 of ACP: constants $a\left(a \in \mathrm{A}_{\delta}\right)$ have been replaced by constants $\tilde{a}$, and in addition to that certain variables $x$ have been replaced by $x+\tilde{\delta}$ in CM2SA and CM3SA. Recall that $x+\tilde{\delta}=x$ if $x \neq \dot{\delta}$, and $\dot{\delta}+\tilde{\delta}=\tilde{\delta}$. This means that $x+\tilde{\delta}$ never stands for $\dot{\delta}$. Axioms SACM1 and SACM2 represent the interaction of absolute delay with left merge. Axiom SACM2 shows that if two parallel processes start to perform actions by performing an action of one of them and that process starts to perform actions at a certain time, only the part of the other process proceeds that starts to perform actions at the same time or later. What happens if such a part does not exist, is reflected more clearly by a generalization of axiom SACM1 than by that axiom itself. This generalization, which is derivable from the axioms of $\mathrm{ACP}^{\mathrm{sat}}$, is equation SACM1' given in Table 6 . Note that a term of the form $v_{\text {abs }}^{p}(y)$ stands for an arbitrary process that starts to perform actions before time $p$; and that a term of the form $\sigma_{\text {abs }}^{p}\left(\nu_{\text {abs }}(z)+\tilde{\delta}\right)$ stands for an arbitrary process that starts to perform actions at time $p$ or deadlocks at time $p$. So equation SACM1' expresses that if the process that would perform the first action can only do so after the ultimate time to start performing actions or to deadlock for the other process, the result will be a deadlock at this ultimate starting time. Note that in case of sequential processes, the process that would first perform actions can always do so, irrespective of the ultimate starting time for the other process. This difference is apparent from equation SAT4', given in Table 6 , which is derivable from the axioms of $\mathrm{ACP}^{\text {sat }}$ and the standard initialization axioms SI13 and SI16 (Table 14, page 22). Axioms SACM3-SACM5 represent the interaction of absolute delay with communication merge. Axioms SACM4 and SACM5 are similar to axioms SACM1 and SACM2. Axiom SACM3 is needed as well because communication merge requires that both processes concerned start performing actions at the same time. Axiom SACM5 is simpler than axiom SACM2 just because of the left distributivity of the communication merge (axiom CM9). Equations SACM3' 


\begin{tabular}{|c|c|}
\hline$\tilde{a} \mid \tilde{b}=\tilde{c} \quad$ if $\gamma(a, b)=c$ & CF1SA \\
\hline$\tilde{a} \mid \tilde{b}=\tilde{\delta} \quad$ if $\gamma(a, b)$ undefined & CF2SA \\
\hline$x \| y=(x \Downarrow y+y \llbracket x)+x \mid y$ & CM1 \\
\hline$\dot{\delta} \Perp x=\dot{\delta}$ & CMID1 \\
\hline$x \Perp \dot{\delta}=\dot{\delta}$ & CMID2 \\
\hline$\tilde{a} \Perp(x+\tilde{\delta})=\tilde{a} \cdot(x+\tilde{\delta})$ & CM2SA \\
\hline$\tilde{a} \cdot x \Perp(y+\tilde{\delta})=\tilde{a} \cdot(x \|(y+\tilde{\delta}))$ & CM3SA \\
\hline$\sigma_{\mathrm{abs}}^{r}(x) \amalg\left(\nu_{\mathrm{abs}}(y)+\tilde{\delta}\right)=\tilde{\delta}$ & SACM1 \\
\hline$\sigma_{\mathrm{abs}}^{p}(x) \amalg\left(v_{\mathrm{abs}}^{p}(y)+\sigma_{\mathrm{abs}}^{p}(z)\right)=\sigma_{\mathrm{abs}}^{p}(x \amalg z)$ & SACM2 \\
\hline$(x+y) \llbracket z=x \llbracket z+y \llbracket z$ & $\mathrm{CM} 4$ \\
\hline$\dot{\delta} \mid x=\dot{\delta}$ & CMID3 \\
\hline$x \mid \dot{\delta}=\dot{\delta}$ & CMID4 \\
\hline$\tilde{a} \cdot x \mid \tilde{b}=(\tilde{a} \mid \tilde{b}) \cdot x$ & CM5SA \\
\hline$\tilde{a} \mid \tilde{b} \cdot x=(\tilde{a} \mid \tilde{b}) \cdot x$ & CM6SA \\
\hline$\tilde{a} \cdot x \mid \tilde{b} \cdot y=(\tilde{a} \mid \tilde{b}) \cdot(x \| y)$ & CM7SA \\
\hline$\left(\nu_{\mathrm{abs}}(x)+\tilde{\delta}\right) \mid \sigma_{\mathrm{abs}}^{r}(y)=\tilde{\delta}$ & SACM3 \\
\hline$\sigma_{\mathrm{abs}}^{r}(x) \mid\left(\nu_{\mathrm{abs}}(y)+\tilde{\delta}\right)=\tilde{\delta}$ & SACM4 \\
\hline$\sigma_{\mathrm{abs}}^{p}(x) \mid \sigma_{\mathrm{abs}}^{p}(y)=\sigma_{\mathrm{abs}}^{p}(x \mid y)$ & SACM5 \\
\hline$(x+y)|z=x| z+y \mid z$ & CM8 \\
\hline$x|(y+z)=x| y+x \mid z$ & CM9 \\
\hline$\partial_{H}(\dot{\delta})=\dot{\delta}$ & D0 \\
\hline$\partial_{H}(\tilde{a})=\tilde{a} \quad$ if $a \notin H$ & D1SA \\
\hline$\partial_{H}(\tilde{a})=\widetilde{\delta} \quad$ if $a \in H$ & D2SA \\
\hline$\partial_{H}\left(\sigma_{\mathrm{abs}}^{p}(x)\right)=\sigma_{\mathrm{abs}}^{p}\left(\partial_{H}(x)\right)$ & $\mathrm{SAD}$ \\
\hline$\partial_{H}(x+y)=\partial_{H}(x)+\partial_{H}(y)$ & D3 \\
\hline$\partial_{H}(x \cdot y)=\partial_{H}(x) \cdot \partial_{H}(y)$ & $\mathrm{D} 4$ \\
\hline$\nu_{\mathrm{abs}}(\dot{\delta})=\dot{\delta}$ & SAU0 \\
\hline$\nu_{\mathrm{abs}}(\tilde{a})=\tilde{a}$ & SAU1 \\
\hline$\nu_{\mathrm{abs}}\left(\sigma_{\mathrm{abs}}^{r}(x)\right)=\tilde{\delta}$ & SAU2 \\
\hline$\nu_{\mathrm{abs}}(x+y)=\nu_{\mathrm{abs}}(x)+\nu_{\mathrm{abs}}(y)$ & SAU3 \\
\hline$\nu_{\mathrm{abs}}(x \cdot y)=\nu_{\mathrm{abs}}(x) \cdot y$ & SAU4 \\
\hline
\end{tabular}

Table 5: Additional axioms for $\operatorname{ACP}^{s a t}\left(a, b \in \mathrm{A}_{\delta}, c \in \mathrm{A}, p \geq 0, r>0\right)$

and SACM4' given in Table 6 generalize axioms SACM3 and SACM4 like equation SACM1' generalizes axiom SACM1. Axiom SAD represents the (lack of) interaction of absolute delay with encapsulation. Axioms SAU0-SAU4 reflect the intended meaning of the urgent initialization operator clearly.

\begin{tabular}{ll}
\hline$\sigma_{\mathrm{abs}}^{p+r}(x) \cdot\left(v_{\mathrm{abs}}^{p}(y)+\sigma_{\mathrm{abs}}^{p}\left(\nu_{\mathrm{abs}}(z)+\tilde{\delta}\right)\right)=\sigma_{\mathrm{abs}}^{p+r}(x \cdot \dot{\delta})$ & $\mathrm{SAT4}^{\prime}$ \\
$\sigma_{\mathrm{abs}}^{p+r}(x) \llbracket\left(v_{\mathrm{abs}}^{p}(y)+\sigma_{\mathrm{abs}}^{p}\left(\nu_{\mathrm{abs}}(z)+\tilde{\delta}\right)\right)=\sigma_{\mathrm{abs}}^{p}(\tilde{\delta})$ & $\mathrm{SACM1}^{\prime}$ \\
$\sigma_{\mathrm{abs}}^{p}\left(\nu_{\mathrm{abs}}(x)+\tilde{\delta}\right) \mid \sigma_{\mathrm{abs}}^{p+r}(y)=\sigma_{\mathrm{abs}}^{p}(\tilde{\delta})$ & $\mathrm{SACM}^{\prime}$ \\
$\sigma_{\mathrm{abs}}^{p+r}(x) \mid \sigma_{\mathrm{abs}}^{p}\left(\nu_{\mathrm{abs}}(y)+\tilde{\delta}\right)=\sigma_{\mathrm{abs}}^{p}(\tilde{\delta})$ & $\mathrm{SACM}^{\prime}$ \\
\hline
\end{tabular}

Table 6: Some derivable equations $(p \geq 0, r>0)$ 
We can prove that the operators $\|\|,, \mid, \partial_{H}$ and $\nu_{\text {abs }}$ can be eliminated in closed terms of $\mathrm{ACP}^{\text {sat }}$. Because of the elimination result for $\mathrm{BPA}^{\text {sat }}$, we are permitted to use induction on the structure of basic terms over $\mathrm{BPA}^{\text {sat }}$ to prove statements for all closed terms of $\mathrm{ACP}^{\mathrm{sat}}$.

Examples We give some examples of a closed term of $\mathrm{ACP}^{\text {sat }}$ and the corresponding basic term (in case $\gamma(a, b)$ and $\gamma(a, c)$ are undefined):

$$
\begin{aligned}
& \sigma_{\text {abs }}^{5.1}(\tilde{a}) \| \sigma_{\text {abs }}^{5.1}(\tilde{b}) \cdot \sigma_{\text {abs }}^{4.9}(\tilde{c})=\sigma_{\text {abs }}^{5.1}(\tilde{a} \cdot \tilde{b} \cdot \dot{\delta}+\tilde{b} \cdot \dot{\delta}) \\
& \sigma_{\text {abs }}^{5.1}(\tilde{a}) \| \sigma_{\text {abs }}^{4.9}(\tilde{b}) \cdot \sigma_{\text {abs }}^{5.1}(\tilde{c})=\sigma_{\text {abs }}^{4.9}\left(\tilde{b} \cdot \sigma_{\text {abs }}^{0.2}(\tilde{a} \cdot \tilde{c}+\tilde{c} \cdot \tilde{a})\right) \\
& \sigma_{\text {abs }}^{5}(\tilde{a}) \| \sigma_{\text {abs }}^{4.9}(\tilde{b}) \cdot \sigma_{\text {abs }}^{5.1}(\tilde{c})=\sigma_{\text {abs }}^{4.9}\left(\tilde{b} \cdot \sigma_{\mathrm{abs}}^{0.1}\left(\tilde{a} \cdot \sigma_{\mathrm{abs}}^{0.1}(\tilde{c})\right)\right) \\
& \nu_{\mathrm{abs}}\left(\sigma_{\mathrm{abs}}^{5.1}(\tilde{a}) \| \sigma_{\mathrm{abs}}^{4.9}(\tilde{b}) \cdot \sigma_{\mathrm{abs}}^{5.1}(\tilde{c})\right)=\tilde{\delta}
\end{aligned}
$$

Semantics of $\mathrm{ACP}^{\mathrm{sat}}$ The structural operational semantics of $\mathrm{ACP}^{\mathrm{sat}}$ is described by the rules for $\mathrm{BPA}^{\text {sat }}$ and the rules given in Table 7 .

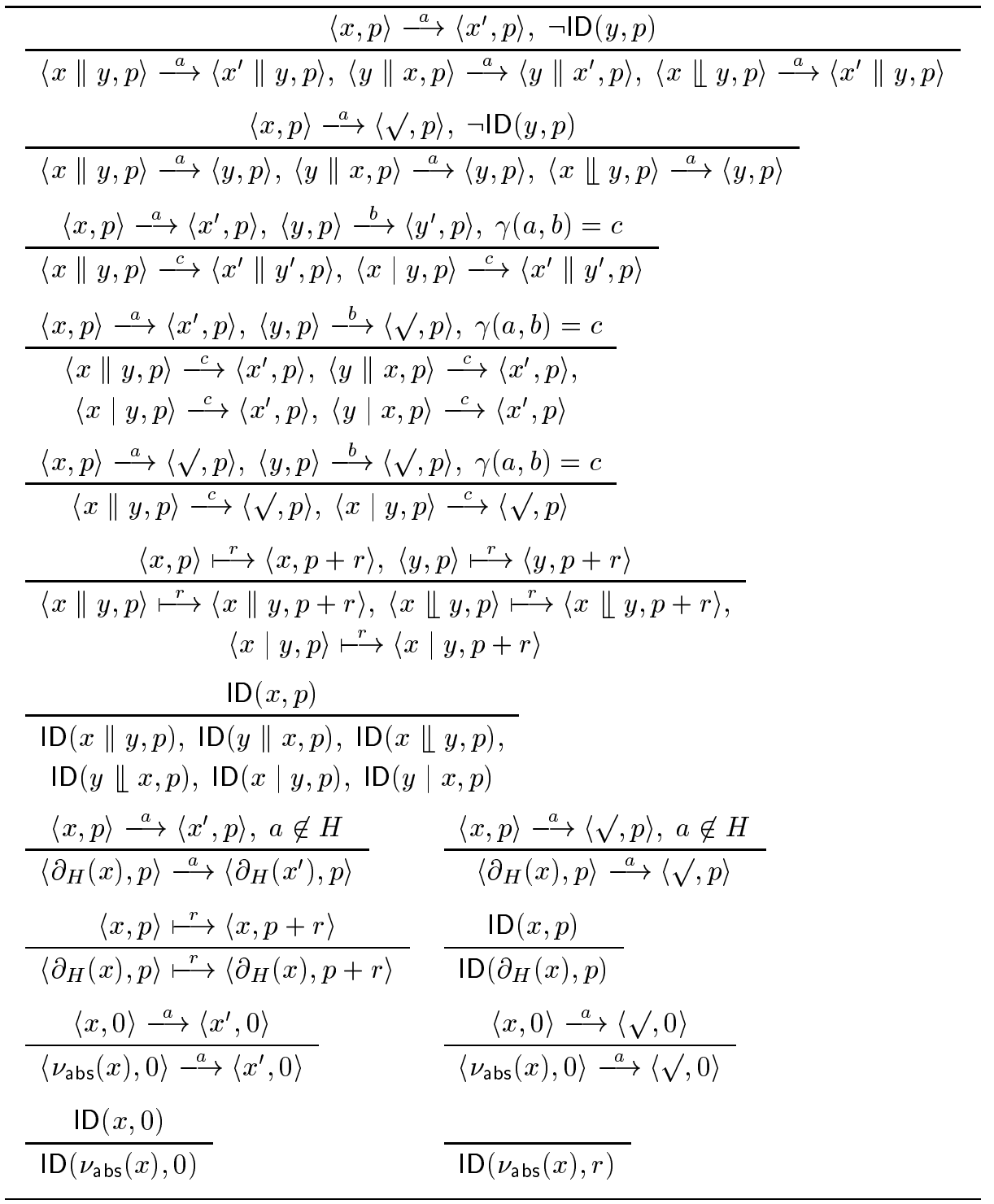

Table 7: Additional rules for $\operatorname{ACP}^{\text {sat }}(a, b, c \in \mathrm{A}, r>0, p \geq 0)$ 
These rules are easy to understand. We will only mention that the first two rules for the parallel composition operator $(\|)$ express that a process $x$ loses all its action related capabilities at time $p$ if it is put in parallel with a process $y$ that can not reach time $p$, and that the last rule for this operator expresses that in such cases the parallel composition can not reach time $p$ either. As in the case of $\mathrm{BPA}^{\text {sat }}$, we obtain a term deduction system in panth format that is stratifiable, so bisimulation equivalence is also a congruence for the additional operators of $\mathrm{ACP}^{\mathrm{sat}}$. Therefore, these operators can be defined on the set of bisimulation equivalence classes as well. As in the case of $\mathrm{BPA}^{\text {sat }}$, we can prove that this results in a model for $\mathrm{ACP}^{\mathrm{sat}}$.

\subsection{Time-stamped actions}

The real time versions $\mathrm{ACP} \rho, \operatorname{ACPr} \rho$ and $\mathrm{ACP} \rho \sigma[2,4]$ feature time-stamped actions - and thus combine execution of actions and passage of time. The time-stamped actions defined below are more closely related to the ones in $\operatorname{ACPs} \rho$ [6], the real time version of $\mathrm{ACP}$ from which $\mathrm{ACP}^{\mathrm{sat}}$ originates. This is because $\mathrm{ACPs} \rho$, like $\mathrm{ACP}^{\mathrm{sat}}$ and unlike $\operatorname{ACP} \rho, \operatorname{ACPr} \rho$ and $\operatorname{ACP} \rho \sigma$, does not exclude the possibility of two or more actions to be performed consecutively at the same point in time.

Time-stamped actions are defined in terms of urgent actions and the delay operator in Table 8. We also define a time-stamped version of immediate deadlock. In ACP $\rho$

\begin{tabular}{l}
\hline$\tilde{a}(p)=\sigma_{\mathrm{abs}}^{p}(\tilde{a})$ \\
$\dot{\delta}(p)=\sigma_{\mathrm{abs}}^{p}(\dot{\delta})$ \\
\hline
\end{tabular}

Table 8: Definitions of time-stamped actions and immediate deadlock $\left(a \in \mathrm{A}_{\delta}, p \geq 0\right)$

and $\operatorname{ACPr} \rho$, which exclude the possibility of two or more actions to be performed consecutively at the same point in time, there is no reason to distinguish, for instance, between the processes $a(p+r) \cdot \delta(p+r)$ and $a(p+r) \cdot b(p)(r>0)$. In ACP sat, unlike in ACPs $\rho$, distinction is made in comparable cases by introducing immediate deadlock to deal with timing inconsistencies. Therefore, we have to introduce time-stamped immediate deadlock here.

We now consider the signature of $\mathrm{BPA}^{\text {sat }}$ with time-stamped actions, i.e. the signature of $\mathrm{BPA}^{\text {sat }}$, but with the urgent action constants $\tilde{a}$, the urgent deadlock constant $\tilde{\delta}$, the immediate deadlock constant $\dot{\delta}$ and the delay operator $\sigma_{\text {abs }}$ replaced by the time-stamped action constants $\tilde{a}(p)$ and the time-stamped deadlock constants $\tilde{\delta}(p)$ and $\dot{\delta}(p)$. From the axioms of $\mathrm{BPA}^{\text {sat }}$ and the definitions of time-stamped actions and immediate deadlock, we can easily derive the equations given in Table 9 for closed terms. Axioms A1-A5 from Table 1 and the equations from Table 9 together can be considered to form the axioms of $\mathrm{BPA}^{\text {sat }}$ with time-stamped actions. The differences with the axioms of BPAs $\rho \delta$ in [6] are all due to the different treatment of timing inconsistencies. Extension of this version to ACP is left to the reader.

\section{Extension of $\mathrm{ACP}^{\mathrm{sat}}$}

In this section, we describe the extension of $\mathrm{ACP}^{\text {sat }}$ with integration and initial abstraction. The extension with integration is needed to be able to embed discrete time 


\begin{tabular}{ll}
\hline$x+\dot{\delta}(0)=x$ & A6TSIDa \\
$\tilde{\delta}(p)+\dot{\delta}(p)=\tilde{\delta}(p)$ & A6TSIDb \\
$\dot{\delta}(p) \cdot x=\dot{\delta}(p)$ & A7TSID \\
$\tilde{a}(p) \cdot x=\tilde{a}(p) \cdot \bar{v}_{\text {abs }}^{p}(x)$ & SATTS \\
$\tilde{a}(p)+\tilde{\delta}(p)=\tilde{a}(p)$ & A6TSa \\
$\tilde{\delta}(p+r)+\tilde{\delta}(p)=\tilde{\delta}(p+r)$ & A6TSb \\
$\tilde{\delta}(p) \cdot x=\tilde{\delta}(p)$ & A7TS \\
$\bar{v}_{\text {abs }}^{p}(\dot{\delta}(p+q))=\dot{\delta}(p+q)$ & SATSI1 \\
$\bar{v}_{\text {abs }}^{p+r}(\dot{\delta}(p))=\dot{\delta}(p+r)$ & SATSI2 \\
$\bar{v}_{\text {abs }}^{p}(\tilde{a}(p+q))=\tilde{a}(p+q)$ & SATSI3 \\
$\bar{v}_{\text {abs }}^{p+r}(\tilde{a}(p))=\dot{\delta}(p+r)$ & SATSI4 \\
$\bar{v}_{\text {ass }}^{p}(x+y)=\bar{v}_{\text {abs }}^{p}(x)+\bar{v}_{\text {abs }}^{p}(y)$ & SATSI5 \\
$\bar{v}_{\text {abs }}^{p}(x \cdot y)=\bar{v}_{\text {abs }}^{p}(x) \cdot y$ & SATSI6 \\
\hline
\end{tabular}

Table 9: Additional axioms for time-stamped actions $\left(a \in \mathrm{A}_{\delta}, p, q \geq 0, r>0\right)$

process algebras, as exemplified in Section 5.3. The extension with initial abstraction is needed to be able to embed process algebras with relative timing, as illustrated in Section 4.3.

Integration and initial abstraction are both variable binding operators. Following e.g. [24], we will introduce variable binding operators by a declaration of the form $f: S_{11}, \ldots, S_{1 k_{1}} . S_{1} \times \ldots \times S_{n 1}, \ldots, S_{n k_{n}} . S_{n} \rightarrow S$. Hereby is indicated that $f$ combines an operator $f^{*}:\left(\left(S_{11} \times \ldots \times S_{1 k_{1}}\right) \rightarrow S_{1}\right) \times \ldots \times\left(\left(S_{n 1} \times \ldots \times S_{n k_{n}}\right) \rightarrow\right.$ $\left.S_{n}\right) \rightarrow S$ with $\lambda$-calculus-like functional abstraction, binding $k_{i}$ variables ranging over $S_{i 1}, \ldots, S_{i k_{i}}$ in the $i$ th argument $(0 \leq i \leq n)$. Applications of $f$ have the following form: $f\left(x_{11}, \ldots, x_{1 k_{1}} . t_{1}, \ldots, x_{n 1}, \ldots, x_{n k_{n}} . t_{n}\right)$, where each $x_{i j}$ is a variable of sort $S_{i j}$ and each $t_{i}$ is a term of sort $S_{i}$.

Integration requires a more extensive theory of the non-negative real numbers than the minimal theory sketched at the beginning of Section 2 (page 4). In the first place, it has to include a theory of sets of non-negative real numbers that makes it possible to deal with set membership and set equality. Besides, the theory should cover suprema of sets of non-negative real numbers.

First, in Section 3.1, ACPsat is extended with integration. After that, in Section 3.2, initial abstraction is added. Finally, some useful additional axioms, derivable for closed terms, are given in Section 3.3.

\subsection{Integration}

We add the integration operator $\int$ to $\mathrm{ACP}^{\text {sat }}$. It provides for alternative composition over a continuum of alternatives. That is, $\int_{v \in V} P$, where $v$ is a variable ranging over $\mathbb{R}_{\geq 0}, V \subseteq \mathbb{R}_{\geq 0}$ and $P$ is a term that may contain free variables, proceeds as one of the alternatives $P[p / v]$ for $p \in V$. The resulting theory is called $\mathrm{ACP}^{\text {sat }}$ I. Obviously, we could first have added integration to $\mathrm{BPA}^{\text {sat }}$, resulting in $\mathrm{BPA}^{\text {sat }} \mathrm{I}$, and then have extended BPA ${ }^{\text {sat }} \mathrm{I}$ to deal with parallelism and communication.

Signature of $\mathbf{A C P}^{\mathrm{sat}} \mathbf{I}$ The signature of $\mathrm{ACP}^{\mathrm{sat}} \mathrm{I}$ is the signature of $\mathrm{ACP}^{\text {sat }}$ extended with the integration (variable-binding) operator $\int: \mathcal{P}\left(\mathbb{R}_{\geq 0}\right) \times \mathbb{R}_{\geq 0} . \mathrm{P} \rightarrow \mathrm{P}$. 
We assume that an infinite set of time variables ranging over $\mathbb{R}_{\geq 0}$ has been given, and denote them by $v, w, \ldots$. Furthermore, we use $V, W, \ldots$ to denote subsets of $\mathbb{R}_{>0}$. We denote terms of $\mathrm{ACP}^{\mathrm{sat}} \mathrm{I}$ by $P, Q, \ldots$.. We will use the following notational convention. We write $\int_{v \in V} P$ for $\int(V, v \cdot P)$.

Axiom system of $\mathbf{A C P}^{\text {sat }} \mathbf{I}$ The axiom system of $\mathrm{ACP}^{\mathrm{sat}}$ I consists of the axioms of $\mathrm{ACP}^{\text {sat }}$ and the equations given in Table 10.

\begin{tabular}{|c|c|}
\hline $\int_{w \in V} R=\int_{v \in V} R[v / w]$ & INT1 \\
\hline $\int_{v \in \emptyset} P=\dot{\delta}$ & INT2 \\
\hline $\int_{v \in\{p\}} P=P[p / v]$ & INT3 \\
\hline $\int_{v \in V \cup W} P=\int_{v \in V} P+\int_{v \in W} P$ & INT4 \\
\hline$V \neq \emptyset \Rightarrow \int_{v \in V} R=R$ & INT5 \\
\hline$(\forall p \in V \bullet P[p / v]=Q[p / v]) \Rightarrow \int_{v \in V} P=\int_{v \in V} Q$ & INT6 \\
\hline$V \neq \emptyset \Rightarrow \int_{v \in V} \sigma_{\mathrm{abs}}^{v}(\dot{\delta})=\sigma_{\mathrm{abs}}^{\mathrm{sup} V}(\dot{\delta})$ & INT7 \\
\hline$V \neq \emptyset, \sup V \notin V \Rightarrow \int_{v \in V} \sigma_{\text {abs }}^{v}(\tilde{\delta})=\sigma_{\text {abs }}^{\text {sup } V}(\dot{\delta})$ & INT8 \\
\hline $\sup V \in V \Rightarrow \int_{v \in V} \sigma_{\mathrm{abs}}^{v}(\tilde{\delta})=\sigma_{\mathrm{abs}}^{\sup V}(\tilde{\delta})$ & INT9 \\
\hline $\int_{v \in V} \sigma_{\mathrm{abs}}^{p}(P)=\sigma_{\mathrm{abs}}^{p}\left(\int_{v \in V} P\right)$ if $p \neq v$ & INT10 \\
\hline $\int_{v \in V}(P+Q)=\int_{v \in V} P+\int_{v \in V} Q$ & INT11 \\
\hline $\int_{v \in V}(P \cdot R)=\left(\int_{v \in V} P\right) \cdot R$ & INT12 \\
\hline $\int_{v \in V}(P \amalg R)=\left(\int_{v \in V} P\right) \amalg R$ & INT13 \\
\hline $\int_{v \in V}(P \mid R)=\left(\int_{v \in V} P\right) \mid R$ & INT14 \\
\hline $\int_{v \in V}(R \mid P)=R \mid\left(\int_{v \in V} P\right)$ & INT15 \\
\hline $\int_{v \in V} \partial_{H}(P)=\partial_{H}\left(\int_{v \in V} P\right)$ & INT16 \\
\hline$v_{\mathrm{abs}}^{p}\left(\int_{v \in V} P\right)=\int_{v \in V} v_{\mathrm{abs}}^{p}(P)$ if $p \neq v$ & SATO6 \\
\hline $\bar{v}_{\mathrm{abs}}^{p}\left(\int_{v \in V} P\right)=\int_{v \in V} \bar{v}_{\mathrm{abs}}^{p}(P)$ if $p \neq v$ & SAI6 \\
\hline$\nu_{\mathrm{abs}}\left(\int_{v \in V} P\right)=\int_{v \in V} \nu_{\mathrm{abs}}(P)$ & SAU5 \\
\hline
\end{tabular}

Table 10: Axioms for integration $(p \geq 0, v$ not free in $R$ )

Axiom INT1 is similar to the $\alpha$-conversion rule of $\lambda$-calculus. Axioms INT2-INT6 are the crucial axioms of integration. They reflect the informal explanation that $\int_{v \in V} P$ proceeds as one of the alternatives $P[p / v]$ for $p \in V$. The remaining axioms are all easily understood by realizing that $\int$ stands for an infinite alternative composition.

We can prove that the auxiliary operators $v_{\text {abs }}$ and $\bar{v}_{\text {abs }}$, as well as sequential compositions in which the form of the first operand is not $\widetilde{a}(a \in \mathrm{A})$ and alternative compositions in which the form of the first operand is $\sigma_{\mathrm{abs}}^{p}(t)$, can be eliminated in closed terms of $\mathrm{BPA}^{\text {sat I }}$ with a restricted form of integration. Basically, this restriction means that in terms of the form $\int_{v \in V} P, V$ is an interval of which the bounds are given by linear expressions over time variables and $P$ is of the form $\sigma_{\mathrm{abs}}^{v}(\tilde{a})$ or $\sigma_{\mathrm{abs}}^{v}(\tilde{a}) \cdot t(a \in$ $\left.A_{\delta}\right)$. This restricted form of integration is essentially the same as prefix integration from [29] (see also [22, 23]). The terms that remain after exhaustive elimination are called the basic terms over $\mathrm{BPA}^{\text {sat }}$ with restricted integration. We can also prove that the operators $\|\|,, \mid, \partial_{H}$ and $\nu_{\text {abs }}$ can be eliminated in closed terms of ACPsat with restricted integration. Because of these elimination results, we are permitted to use induction on the structure of basic terms over $\mathrm{BPA}^{\text {sat }}$ with restricted integration to prove statements for all closed terms of $\mathrm{ACP}^{\text {sat }}$ with restricted integration. 
Examples We give some examples of a closed term of $\mathrm{ACP}^{\mathrm{sat}}$ with restricted integration and the corresponding basic term:

$$
\begin{aligned}
& \int_{v \in[4.9,5.1)} \sigma_{\mathrm{abs}}^{v}\left(\nu_{\mathrm{abs}}\left(\sigma_{\mathrm{abs}}^{0.9}(\tilde{a}) \| \sigma_{\mathrm{abs}}^{1.8}(\tilde{b}) \cdot \sigma_{\mathrm{abs}}^{2.7}(\tilde{c})\right)\right)=\sigma_{\mathrm{abs}}^{5.1}(\dot{\delta}) \\
& \int_{v \in[4.9,5.1)} \sigma_{\mathrm{abs}}^{v}(\tilde{a})+\int_{v \in[4.9,5.1)} \sigma_{\mathrm{abs}}^{v}(\tilde{b})=\int_{v \in[4.9,5.1)} \sigma_{\mathrm{abs}}^{v}(\tilde{a}+\tilde{b}) \\
& \left(\int_{v \in[4.9,5.1)} \sigma_{\mathrm{abs}}^{v}(\tilde{a})\right) \mid\left(\int_{v \in[4.9,5.1)} \sigma_{\mathrm{abs}}^{v}(\tilde{b})\right)=\int_{v \in[4.9,5.1)} \sigma_{\mathrm{abs}}^{v}(\tilde{c}) \quad \text { if } \gamma(a, b)=c \\
& \left(\int_{v \in[4.9,5.1)} \sigma_{\mathrm{abs}}^{v}(\tilde{a})\right) \mid\left(\int_{v \in[4.9,5.1)} \sigma_{\mathrm{abs}}^{v}(\tilde{b})\right)=\int_{v \in[4.9,5.1)} \sigma_{\mathrm{abs}}^{v}(\tilde{\delta}) \quad \text { if } \gamma(a, b) \text { undefined }
\end{aligned}
$$

Semantics of $\mathrm{ACP}^{\mathrm{sat}} \mathbf{I}$ The structural operational semantics of $\mathrm{ACP}^{\mathrm{sat}} \mathrm{I}$ is described by the rules for $\mathrm{ACP}^{\text {sat }}$ and the rules given in Table 11 .

\begin{tabular}{cc}
\hline$\frac{\langle P[q / v], p\rangle \stackrel{a}{\longrightarrow}\left\langle P^{\prime}, p\right\rangle, q \in V}{\left\langle\int_{v \in V} P, p\right\rangle \stackrel{a}{\longrightarrow}\left\langle P^{\prime}, p\right\rangle}$ & $\frac{\langle P[q / v], p\rangle \stackrel{a}{\longrightarrow}\langle\sqrt{ }, p\rangle, q \in V}{\left\langle\int_{v \in V} P, p\right\rangle \stackrel{a}{\longrightarrow}\langle\sqrt{ }, p\rangle}$ \\
$\frac{\langle P[q / v], p\rangle \stackrel{r}{\longmapsto}\langle P[q / v], p+r\rangle, q \in V}{\left\langle\int_{v \in V} P, p\right\rangle \stackrel{r}{\longmapsto}\left\langle\int_{v \in V} P, p+r\right\rangle}$ & $\frac{\operatorname{ID}(P[q / v], p) \text { for all } q \in V}{\operatorname{ID}\left(\int_{v \in V} P, p\right)}$ \\
\hline
\end{tabular}

Table 11: Rules for integration $(a \in \mathrm{A}, r>0, p, q \geq 0)$

The rules for integration are simple generalizations of the rules for alternative composition to the infinite case.

The panth format does not cover variable binding operators such as integration. The integration operator combines an ordinary operator $\int^{*}: \mathcal{P}\left(\mathbb{R}_{\geq 0}\right) \times\left(\mathbb{R}_{\geq 0} \rightarrow \mathrm{P}\right) \rightarrow$ $P$ with $\lambda$-calculus-like functional abstraction, binding one variable ranging over $\mathbb{R}_{\geq 0}$ in the second argument. This implies that we have additional sorts here, including the sort of functions from non-negative real numbers to processes. These functions, denoted by closed terms of the form $v . P$, where $v$ may occur free in $P$, cannot be dealt with in the same way as the non-negative real numbers: (1) they are denoted using open terms of the sort of processes and (2) their application is syntactically represented by means of substitution. As for (1), we can define bisimulation equivalence on closed terms $v . P$ and $w . Q$ as well as on open terms $P$ and $Q$ in such a way that it corresponds

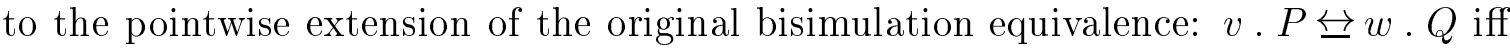

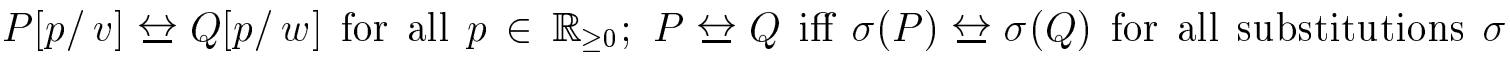
of non-negative real numbers for time variables. As for (2), we have to make a clear distinction between variables that range over a semantic domain, such as $v$ in the rules for integration, and meta-variables that range over a syntactic domain, such as $P$ and $P^{\prime}$ in the rules for integration. In [24], the former variables are called actual variables and the latter ones formal variables. Careful checking of the proof of the congruence theorem for the panth format given in [43] shows that the result goes through for the case with variable binding operators if the condition about the number of constants and operators in the first argument of a conclusion is as in the many-sorted case without variable binding operators: the number of constants and operators that yield processes is restricted to zero or one.

The signature of $\mathrm{ACP}^{\mathrm{sat}} \mathrm{I}$ together with the rules for the operational semantics of $\mathrm{ACP}^{\mathrm{sat}} \mathrm{I}$ constitute a stratifiable term deduction system in this generalized panth format, so bisimulation equivalence is also a congruence for the integration operator. Hence, this operator can be defined on the set of bisimulation equivalence classes as well. As in the case of $\mathrm{BPA}^{\text {sat }}$ and $\mathrm{ACP}^{\mathrm{sat}}$, we can prove that this results in a model for $\mathrm{ACP}^{\mathrm{sat}} \mathrm{I}$. We will call this model $\mathrm{M}_{\mathrm{A}}$. 


\subsection{Initial abstraction}

We add the initial abstraction operator $\sqrt{s}_{\mathrm{s}}$ to $\mathrm{ACP}^{\mathrm{sat}} \mathrm{I}$. It provides for (simple) parametric timing: $\sqrt{\mathrm{s}}_{\mathrm{s}} v \cdot F$, where $v$ is a variable ranging over $\mathbb{R}_{\geq 0}$ and $F$ is a term that may contain free variables, proceeds as $F[p / v]$ if initialized at time $p \in \mathbb{R}_{\geq 0}$. This means that $\sqrt{s}_{\mathrm{s}} v . F$ denotes a function $f: \mathbb{R}_{\geq 0} \rightarrow \mathrm{P}$ that satisfies $f(p)=\bar{v}_{\text {abs }}^{p}(f(p))$ for all $p \in \mathbb{R}_{\geq 0}$. In the resulting theory, called $A C P^{s a t} \mathrm{I} \checkmark$, the sort $\mathrm{P}$ of processes is replaced by the sort $\mathrm{P}^{*}$ of parametric time processes. Of course, it is also possible to add the initial abstraction operator to $\mathrm{ACP}^{\text {sat }}$, resulting in a theory $\mathrm{ACP}^{\mathrm{sat}} \sqrt{ }$.

Signature of $\mathbf{A C P}^{\mathrm{sat}} \mathbf{I} \sqrt{ }$ The signature of $\mathrm{ACP}^{\mathrm{sat}} \mathrm{I} \sqrt{ }$ is the signature of $\mathrm{ACP}^{\mathrm{sat}} \mathrm{I}$ extended with the initial abstraction (variable-binding) operator $\sqrt{s}_{\mathrm{s}}: \mathbb{R}_{\geq 0} . \mathrm{P}^{*} \rightarrow \mathrm{P}^{*}$.

We now use $x, y, \ldots$ to denote variables of sort $\mathrm{P}^{*}$. Terms of ACP ${ }^{\text {sat }} \mathrm{I} \sqrt{ }$ are denoted by $F, G, \ldots$ We will use the following notational convention. We write $\sqrt{s}_{\mathrm{s}} v$. $F$ for $\sqrt{ }(v . F)$.

Axiom system of $\mathbf{A C P}^{\mathrm{sat}} \mathbf{I} \sqrt{ }$ The axiom system of $\mathrm{ACP}^{\mathrm{sat}} \mathrm{I} \sqrt{ }$ consists of the axioms of $\mathrm{ACP}^{\mathrm{sat}} \mathrm{I}$ and the equations given in Table 12 .

\begin{tabular}{|c|c|}
\hline$\sqrt{\mathrm{s}}_{\mathrm{s}} w \cdot G=\sqrt{\mathrm{s}}_{\mathrm{s}} v \cdot G[v / w]$ & SIA1 \\
\hline $\bar{v}_{\mathrm{abs}}^{p}\left(\sqrt{ }_{\mathrm{s}} v \cdot F\right)=\bar{v}_{\mathrm{abs}}^{p}(F[p / v])$ & SIA2 \\
\hline$\sqrt{s}_{\mathrm{s}} v \cdot\left(\sqrt{ }_{\mathrm{s}} w \cdot F\right)=\sqrt{ }_{\mathrm{s}} v \cdot F[v / w]$ & SIA3 \\
\hline$G=\sqrt{ }_{\mathrm{s}} v \cdot G$ & SIA 4 \\
\hline$\left(\forall p \in \mathbb{R}_{\geq 0} \bullet \bar{v}_{\mathrm{abs}}^{p}(x)=\bar{v}_{\mathrm{abs}}^{p}(y)\right) \Rightarrow x=y$ & SIA 5 \\
\hline$\sigma_{\mathrm{abs}}^{p}(\tilde{a}) \cdot x=\sigma_{\mathrm{abs}}^{p}(\tilde{a}) \cdot \bar{v}_{\mathrm{abs}}^{p}(x)$ & SIA6 \\
\hline$\sigma_{\mathrm{abs}}^{p}\left(\sqrt{\mathrm{s}}_{\mathrm{s}} v \cdot F\right)=\sigma_{\mathrm{abs}}^{p}(F[0 / v])$ & SIA7 \\
\hline$\left(\sqrt{ }_{\mathrm{s}} v \cdot F\right)+G=\sqrt{\mathrm{s}}_{\mathrm{s}} v \cdot\left(F+\bar{v}_{\mathrm{abs}}^{v}(G)\right)$ & SIA8 \\
\hline$\left(\sqrt{ }_{\mathrm{s}} v \cdot F\right) \cdot G=\sqrt{ }_{\mathrm{s}} v \cdot(F \cdot G)$ & SIA9 \\
\hline$v_{\mathrm{abs}}^{p}\left(\sqrt{ }_{\mathrm{s}} v \cdot F\right)=\sqrt{ }_{\mathrm{s}} v \cdot v_{\mathrm{abs}}^{p}(F)$ if $p \neq v$ & SIA 10 \\
\hline$\left(\sqrt{\mathrm{s}}_{\mathrm{s}} v \cdot F\right) \amalg G=\sqrt{\mathrm{s}}_{\mathrm{s}} v \cdot\left(F \amalg \bar{v}_{\mathrm{abs}}^{v}(G)\right)$ & SIA11 \\
\hline$G \Perp\left(\sqrt{ }_{\mathrm{s}} v \cdot F\right)=\sqrt{\mathrm{s}}_{\mathrm{s}} v \cdot\left(\bar{v}_{\mathrm{abs}}^{v}(G) \amalg F\right)$ & SIA12 \\
\hline$\left(\sqrt{ }_{\mathrm{s}} v \cdot F\right) \mid G=\sqrt{\mathrm{s}}_{\mathrm{s}} v \cdot\left(F \mid \bar{v}_{\mathrm{abs}}^{v}(G)\right)$ & SIA13 \\
\hline$G \mid\left(\sqrt{ }_{\mathrm{s}} v \cdot F\right)=\sqrt{\mathrm{s}}_{\mathrm{s}} v \cdot\left(\bar{v}_{\mathrm{abs}}^{v}(G) \mid F\right)$ & SIA14 \\
\hline$\partial_{H}\left(\sqrt{ }_{\mathrm{s}} v \cdot F\right)=\sqrt{ }_{\mathrm{s}} v \cdot \partial_{H}(F)$ & SIA15 \\
\hline$\nu_{\mathrm{abs}}\left(\sqrt{\mathrm{s}}_{\mathrm{s}} v \cdot F\right)=\sqrt{\mathrm{s}}_{\mathrm{s}} v \cdot \nu_{\mathrm{abs}}(F)$ & SIA16 \\
\hline $\int_{v \in V}\left(\sqrt{ }_{s} w \cdot F\right)=\sqrt{s}_{s} w \cdot\left(\int_{v \in V} F\right)$ if $v \neq w$ & SIA17 \\
\hline
\end{tabular}

Table 12: Axioms for standard initial abstraction $(p \geq 0, v$ not free in $G)$

Axioms SIA1 and SIA2 are similar to the $\alpha$ - and $\beta$-conversion rules of $\lambda$-calculus. Axiom SIA3 points out that multiple initial abstractions can simply be replaced by one. Axiom SIA4 shows that processes with absolute timing can be treated as special cases of processes with parametric timing: they do not vary with different initialization times. Axiom SIA5 is an extensionality axiom. Axiom SIA6 expresses that in case a process performs an action and then proceeds as another process, the initialization time of the latter process is the time at which the action is performed. Notice that 
the equation $\tilde{a} \cdot x=\tilde{a} \cdot \bar{v}_{\text {abs }}^{0}(x)$ is a special case of axiom SIA6. The related equation $\sigma_{\text {abs }}^{p}(x)=\sigma_{\text {abs }}^{p}\left(\bar{v}_{\text {abs }}^{0}(x)\right)$ follows immediately from axioms SAT1 and SAT2 (Table 2, page 6). Axioms SIA7-SIA17 become easier to understand by realizing that $\sqrt{\mathrm{s}}_{\mathrm{s}} v$. F denotes a function $f: \mathbb{R}_{\geq 0} \rightarrow \mathrm{P}$ such that $f(p)=\bar{v}_{\text {abs }}^{p}(f(p))$ for all $p \in \mathbb{R}_{\geq 0}$. This is reflected by the equation

$$
\sqrt{\mathrm{s}}_{\mathrm{s}} v \cdot F=\sqrt{\mathrm{s}}_{\mathrm{s}} v \cdot \bar{v}_{\mathrm{abs}}^{v}(F) \text { SIAI }
$$

which can be derived using axioms SIA2 and SIA5 and a useful special case of standard initialization axiom SI2 presented in Section 3.3, viz. $\bar{v}_{\mathrm{abs}}^{p}\left(\bar{v}_{\mathrm{abs}}^{p}(x)\right)=\bar{v}_{\mathrm{abs}}^{p}(x)$.

The elimination results for $\mathrm{ACP}^{\mathrm{sat}} \mathrm{I} \checkmark$ with the restricted form of integration mentioned in Section 3.1 are essentially the same as the ones for $\mathrm{ACP}^{\mathrm{sat}}$ I with the restricted form of integration. Besides, all closed terms of $\mathrm{ACP}^{\mathrm{sat}} \mathrm{I} \sqrt{ }$ with this restricted form of integration can be written in the form $\sqrt{ }_{\mathrm{s}} v . F$ where $F$ is a basic term over BPA sat $^{\text {sat }}$ with restricted integration.

Examples We give some examples of a closed term of $\mathrm{ACP}^{\mathrm{sat}} \mathrm{I} \sqrt{ }$ with restricted integration, the corresponding term of the form $\sqrt{s}_{\mathrm{s}} v . F$ where $F$ is a basic term and, if possible, the corresponding basic term without initial abstraction:

$$
\begin{aligned}
& \sqrt{\mathrm{s}}_{\mathrm{s}} v \cdot v_{\mathrm{abs}}^{v+2.3}\left(\sqrt{\mathrm{s}}_{\mathrm{s}} w \cdot \sigma_{\mathrm{abs}}^{w}(\tilde{a})\right)=\sqrt{\mathrm{s}}_{\mathrm{s}} v \cdot \sigma_{\mathrm{abs}}^{v}(\tilde{a}) \\
& \sqrt{\mathrm{s}}_{\mathrm{s}} v \cdot \bar{v}_{\mathrm{abs}}^{v+2.3}\left(\sqrt{\mathrm{s}}_{\mathrm{s}} w \cdot \sigma_{\mathrm{abs}}^{w}(\tilde{a})\right)=\sqrt{\mathrm{s}}_{\mathrm{s}} v \cdot \sigma_{\mathrm{abs}}^{v+2.3}(\tilde{a}) \\
& \bar{v}_{\mathrm{abs}}^{3.9}\left(\sqrt{\mathrm{s}}_{\mathrm{s}} v \cdot \bar{v}_{\mathrm{abs}}^{v+2.3}\left(\int_{w \in[6,6.1)} \sigma_{\mathrm{abs}}^{w}(\tilde{a})\right)=\sqrt{\mathrm{s}}_{\mathrm{s}} v \cdot \sigma_{\mathrm{abs}}^{6.2}(\dot{\delta})=\sigma_{\mathrm{abs}}^{6.2}(\dot{\delta})\right. \\
& \bar{v}_{\mathrm{abs}}^{3.6}\left(\sqrt{\mathrm{s}}_{\mathrm{s}} v \cdot \bar{v}_{\mathrm{abs}}^{v+2.3}\left(\int_{w \in[6,6.1)} \sigma_{\mathrm{abs}}^{w}(\tilde{a})\right)=\sqrt{\mathrm{s}}_{\mathrm{s}} v \cdot \int_{w \in[6,6.1)} \sigma_{\mathrm{abs}}^{w}(\tilde{a})=\int_{w \in[6,6,1)} \sigma_{\mathrm{abs}}^{w}(\tilde{a})\right.
\end{aligned}
$$

On the basis of the rules for its operational semantics, the operators of $\mathrm{ACP}^{\mathrm{sat}} \mathrm{I}$ can also be directly defined on real time transition systems in a straightforward way. In the following, we will describe a model of $\mathrm{ACP}^{\mathrm{sat}} \mathrm{I} \sqrt{ }$ in terms of these operators.

Semantics of $\operatorname{ACP}^{\mathrm{sat}} \mathbf{I} \sqrt{ }$ We have to extend RTTS(A) to the function space

$$
\operatorname{RTTS}^{*}(\mathrm{~A})=\left\{f: \mathbb{R}_{\geq 0} \rightarrow \operatorname{RTTS}(\mathrm{A}) \mid \forall p \in \mathbb{R}_{\geq 0} \bullet f(p)=\bar{v}_{\text {abs }}^{p}(f(p))\right\}
$$

of real time transition systems with parametric timing. We use $f, g, \ldots$ to denote elements of $\operatorname{RTTS}^{*}(\mathrm{~A})$. In Table 13, the constants and operators of ACPsat $\mathrm{I} \checkmark$ are defined on $\operatorname{RTTS}^{*}(\mathrm{~A})$. We use $\lambda$-notation for functions - here $t$ is a variable ranging over $\mathbb{R}_{\geq 0}$. We write $f(t) * g$ for the real time transition system obtained from $f(t)$ by replacing $\langle s, p\rangle \stackrel{a}{\longrightarrow}\langle\sqrt{ }, p\rangle$ by $\langle s, p\rangle \stackrel{a}{\longrightarrow}\left\langle s^{\prime}, p\right\rangle$, where $s^{\prime}$ is the root state of $g(p)$, whenever $s$ is reachable from the root state of $f(t)$.

We say that $f, g \in \mathrm{RTTS}^{*}(\mathrm{~A})$ are bisimilar if for all $p \in \mathbb{R}_{\geq 0}$, there exists a bisimulation $R$ such that $R(f(p), g(p))$. It is easy to see that bisimulation equivalence as defined here is a congruence for the operators of $\mathrm{ACP}^{\mathrm{sat}} \mathrm{I} \checkmark$. We obtain a model of ACP ${ }^{\text {sat }} \mathrm{I} \checkmark$ by defining all operators on the set of bisimulation equivalence classes. We will call this model $\mathrm{M}_{\mathrm{A}}^{*}$. Notice that $f \in \mathrm{RTTS}^{*}(\mathrm{~A})$ corresponds to a process that can be written with the constants and operators of $\mathrm{ACP}^{\text {sat }} \mathrm{I}$ only iff $\bar{v}_{\mathrm{abs}}^{0}(f)=f$. In fact, $\mathrm{M}_{\mathrm{A}}$ is isomorphic to a subalgebra of $\mathrm{M}_{\mathrm{A}}^{*}$. 


\begin{tabular}{l}
\hline$\dot{\delta}=\lambda t \cdot \dot{\delta}$ \\
$\tilde{a}=\lambda t \cdot \bar{v}_{\mathrm{abs}}^{t}(\tilde{a})$ \\
$\sigma_{\mathrm{abs}}^{p}(f)=\lambda t \cdot \bar{v}_{\mathrm{abs}}^{t}\left(\sigma_{\mathrm{abs}}^{p}(f(0))\right)$ \\
$f+g=\lambda t \cdot(f(t)+g(t))$ \\
$f \cdot g=\lambda t \cdot(f(t) * g)$ \\
$v_{\mathrm{abs}}^{p}(f)=\lambda t \cdot \bar{v}_{\mathrm{abs}}^{t}\left(v_{\mathrm{abs}}^{p}(f(t))\right)$ \\
$\bar{v}_{\mathrm{abs}}^{p}(f)=f(p)$ \\
$f \| g=\lambda t \cdot(f(t) \| g(t))$ \\
$f \amalg g=\lambda t \cdot(f(t) \llbracket g(t))$ \\
$f \mid g=\lambda t \cdot(f(t) \mid g(t))$ \\
$\partial_{H}(f)=\lambda t \cdot \partial_{H}(f(t))$ \\
$\nu_{\mathrm{abs}}(f)=\lambda t \cdot \bar{v}_{\mathrm{abs}}^{t}\left(\nu_{\mathrm{abs}}(f(t))\right)$ \\
$\int_{v \in V}(f)=\lambda t \cdot \int_{v \in V}(f(t))$ \\
$\mathcal{V}_{\mathrm{s}}^{*} \varphi=\lambda t \cdot \bar{v}_{\mathrm{abs}}^{t}(\varphi(t))$ \\
\hline
\end{tabular}

Table 13: Definition of operators on $\operatorname{RTTS}^{*}\left(\varphi: \mathbb{R}_{\geq 0} \rightarrow \operatorname{RTTS}^{*}(\mathrm{~A}), a \in \mathrm{A}_{\delta}, p \in \mathbb{R}_{\geq 0}\right)$

\begin{tabular}{|c|c|}
\hline $\bar{v}_{\mathrm{abs}}^{p}\left(v_{\mathrm{abs}}^{p+r}(x)\right)=v_{\mathrm{abs}}^{p+r}\left(\bar{v}_{\mathrm{abs}}^{p}(x)\right)$ & SI1 \\
\hline $\bar{v}_{\mathrm{abs}}^{p}\left(\bar{v}_{\mathrm{abs}}^{p+q}(x)\right)=\bar{v}_{\mathrm{abs}}^{p+q}(x)$ & SI2 \\
\hline $\bar{v}_{\mathrm{abs}}^{p+q}\left(v_{\mathrm{abs}}^{p}(x)\right)=\sigma_{\mathrm{abs}}^{p+q}(\dot{\delta})$ & SI3 \\
\hline$v_{\mathrm{abs}}^{p}\left(\bar{v}_{\mathrm{abs}}^{p+q}(x)\right)=\sigma_{\mathrm{abs}}^{p}(\dot{\delta})$ & SI4 \\
\hline$\sigma_{\mathrm{abs}}^{p}(\dot{\delta})+\bar{v}_{\mathrm{abs}}^{p}(x)=\bar{v}_{\mathrm{abs}}^{p}(x)$ & SI5 \\
\hline$\sigma_{\mathrm{abs}}^{p}(\tilde{\delta})+\bar{v}_{\mathrm{abs}}^{p}(x+\tilde{\delta})=\bar{v}_{\mathrm{abs}}^{p}(x+\tilde{\delta})$ & SI6 \\
\hline $\bar{v}_{\mathrm{abs}}^{r}(x)+\widetilde{\delta}=\bar{v}_{\mathrm{abs}}^{r}(x)$ & SI7 \\
\hline$v_{\mathrm{abs}}^{p}\left(v_{\mathrm{abs}}^{q}(x)\right)=v_{\mathrm{abs}}^{\min }(p, q)(x)$ & SI8 \\
\hline $\bar{v}_{\mathrm{abs}}^{p}\left(\bar{v}_{\mathrm{abs}}^{q}\left(\bar{v}_{\mathrm{abs}}^{q^{\prime}}(x)\right)\right)=\bar{v}_{\mathrm{abs}}^{\max (p, q)}\left(\bar{v}_{\mathrm{abs}}^{q^{\prime}}(x)\right)$ & SI9 \\
\hline $\bar{v}_{\mathrm{abs}}^{p}(x \Downarrow y)=\bar{v}_{\mathrm{abs}}^{p}(x) \amalg \bar{v}_{\mathrm{abs}}^{p}(y)$ & SI10 \\
\hline $\bar{v}_{\mathrm{abs}}^{p}(x \mid y)=\bar{v}_{\mathrm{abs}}^{p}(x) \mid \bar{v}_{\mathrm{abs}}^{p}(y)$ & SI11 \\
\hline $\bar{v}_{\mathrm{abs}}^{p}\left(\partial_{H}(x)\right)=\partial_{H}\left(\bar{v}_{\mathrm{abs}}^{p}(x)\right)$ & SI12 \\
\hline $\bar{v}_{\mathrm{abs}}^{0}\left(\nu_{\mathrm{abs}}(x)\right)=\nu_{\mathrm{abs}}\left(\bar{v}_{\mathrm{abs}}^{0}(x)\right)$ & SI13 \\
\hline $\bar{v}_{\mathrm{abs}}^{r}\left(\nu_{\mathrm{abs}}(x)\right)=\sigma_{\mathrm{abs}}^{r}(\dot{\delta})$ & SI14 \\
\hline$\nu_{\text {abs }}\left(\bar{v}_{\text {abs }}^{r}(x)\right)=\tilde{\delta}$ & SI15 \\
\hline$v_{\mathrm{abs}}^{r}\left(\nu_{\mathrm{abs}}(x)\right)=\nu_{\mathrm{abs}}(x)$ & SI16 \\
\hline$\nu_{\mathrm{abs}}\left(v_{\mathrm{abs}}^{r}(x)\right)=\nu_{\mathrm{abs}}(x)$ & SI17 \\
\hline
\end{tabular}

Table 14: Standard initialization axioms $\left(p, q, q^{\prime} \geq 0, r>0\right)$

\subsection{Standard initialization axioms}

In Table 14, some equations concerning initialization and time-out are given that hold in the model $M_{A}^{*}$, and that are derivable for closed terms of $A_{C P}{ }^{s a t} \mathrm{I} \checkmark$. We will use these axioms in proofs in subsequent sections. Notice that the very useful equation $\bar{v}_{\text {abs }}^{p}\left(\bar{v}_{\text {abs }}^{p}(x)\right)=\bar{v}_{\text {abs }}^{p}(x)$ is a special case of axiom SI2. We can easily prove by means of the standard initialization axioms, using axioms SIA2 and SIA5 (Table 12, page 20), that initial abstraction distributes over,$+\|$,$\| and |:$

$$
\left(\sqrt{ }_{\mathrm{s}} v \cdot F\right) \square\left(\sqrt{\mathrm{s}}_{\mathrm{s}} v \cdot F^{\prime}\right)=\sqrt{\mathrm{s}} v \cdot\left(F \square F^{\prime}\right) \text { DISTR } \square
$$


for $\square=+, \|, \mathbb{L}, \mid$. Using this fact shortens many of the calculations needed in the proof of Theorem 6 (embedding of $\mathrm{ACP}^{\mathrm{srt}}$ in $\mathrm{ACP}^{\mathrm{sat}} \sqrt{ }$ ).

\section{Real time process algebra: relative timing}

In this section, we give the signature, axioms and term model of $\mathrm{ACP}^{\text {srt }}$, a standard real time process algebra with relative timing. $\mathrm{ACP}^{\text {srt }}$ originates from the theory ACPst, presented in [6]. Like ACPst, it separates execution of actions and passage of time.

First, in Section 4.1, we treat $\mathrm{BPA}^{\text {srt }}$, basic standard real time process algebra with relative timing, in which parallelism and communication are not considered. After that, in Section 4.2, $\mathrm{BPA}^{\text {srt }}$ is extended to $\mathrm{ACP}^{\text {srt }}$ to deal with parallelism and communication as well. Finally, we show in Section 4.3 how $\mathrm{ACP}^{\text {srt }}$ can be embedded in $\mathrm{ACP}^{\mathrm{sat}} \sqrt{ }$.

\subsection{Basic process algebra}

In $\mathrm{BPA}^{\text {srt }}$, we have the constants $\tilde{\widetilde{a}}$ and $\tilde{\tilde{\delta}}$ instead of $\tilde{a}$ and $\tilde{\delta}$, and the operator $\sigma_{\text {rel }}$ (relative delay) instead of $\sigma_{\text {abs }}$ (absolute delay). The constants $\tilde{\widetilde{a}}$ and $\tilde{\tilde{\delta}}$ stand for $a$ without any delay and a deadlock without any delay, respectively. The process $\sigma_{\text {rel }}^{p}(x)$ is the process $x$ delayed for a period of time $p$. We also have relative counterparts of the absolute time-out and initialization operators: $v_{\text {rel }}$ (relative time-out) and $\bar{v}_{\text {rel }}$ (relative initialization). The process $v_{\text {rel }}^{p}(x)$ is the part of $x$ that starts to perform actions after a period of time shorter than $p$. The process $\bar{v}_{\text {rel }}^{p}(x)$ is the part of $x$ that starts to perform actions after a period of time longer than or equal to $p$.

The notation $\widetilde{\tilde{a}}$ for urgent actions in case of relative timing was also used in ACPst [6], the theory from which $\mathrm{ACP}^{\text {srt }}$ originates.

Signature of $\mathrm{BPA}^{\text {srt }}$ The signature of $\mathrm{BPA}^{\text {srt }}$ consists of the urgent action constants $\tilde{\tilde{a}}: \rightarrow \mathrm{Pr}^{\mathrm{r}}$ (for each $a \in \mathrm{A}$ ), the urgent deadlock constant $\widetilde{\tilde{\delta}}: \rightarrow \mathrm{P}^{\mathrm{r}}$, the immediate deadlock constant $\dot{\delta}: \rightarrow \mathrm{Pr}^{\mathrm{r}}$, the alternative composition operator $+: \mathrm{Pr}^{\mathrm{r}} \times \mathrm{Pr}^{\mathrm{r}} \rightarrow \mathrm{Pr}^{\mathrm{r}}$, the sequential composition operator $\cdot: \mathrm{Pr}^{\mathrm{r}} \times \mathrm{Pr}^{\mathrm{r}} \rightarrow \mathrm{Pr}^{\mathrm{r}}$, the relative delay operator $\sigma_{\text {rel }}: \mathbb{R}_{\geq 0} \times \mathrm{Pr}^{\mathrm{r}} \rightarrow \mathrm{P}^{\mathrm{r}}$, the relative time-out operator $v_{\text {rel }}: \mathbb{R}_{\geq 0} \times \mathrm{Pr}^{\mathrm{r}} \rightarrow \mathrm{Pr}^{\mathrm{r}}$, and the relative initialization operator $\bar{v}_{\text {rel }}: \mathbb{R}_{\geq 0} \times \mathrm{Pr}^{\mathrm{r}} \rightarrow \mathrm{P}^{\mathrm{r}}$.

Axioms of $\mathrm{BPA}^{\text {srt }}$ The axiom system of $\mathrm{BPA}^{\text {srt }}$ consists of the equations given in Tables 1 and 15 .

The axioms of $\mathrm{BPA}^{\text {srt }}$ are to a large extent simple reformulations of the axioms of $\mathrm{BPA}^{\text {sat }}$. That is, constants $\tilde{a}\left(a \in \mathrm{A}_{\delta}\right)$ have been replaced by constants $\tilde{\tilde{a}}$, and the operators $\sigma_{\text {abs }}, v_{\text {abs }}$ and $\bar{v}_{\text {abs }}$ have been replaced by $\sigma_{\text {rel }}, v_{\text {rel }}$ and $\bar{v}_{\text {rel }}$, respectively. Striking is the replacement of the axioms SAT4, SAT5 and SAT6 by the simple axiom SRT4. This axiom reflects that timing is relative to the most recent execution of an action. Axioms SRI0-SRI5 are reformulations, in the above-mentioned way, of alternative axioms for axioms SAI0-SAI5 - which, unlike axioms SAI0-SAI5, do not accommodate the addition of initial abstraction (see also Section 2.1).

Similar to the case of BPA ${ }^{\text {sat }}$, we can prove that the auxiliary operators $v_{\text {rel }}$ and $\bar{v}_{\text {rel }}$, as well as sequential compositions in which the form of the first operand is not $\tilde{\widetilde{a}}$ 


\begin{tabular}{|c|c|}
\hline$\overline{\sigma_{\text {rel }}^{0}(x)=x}$ & SRT1 \\
\hline$\sigma_{\text {rel }}^{p}\left(\sigma_{\text {rel }}^{q}(x)\right)=\sigma_{\text {rel }}^{p+q}(x)$ & SRT2 \\
\hline$\sigma_{\mathrm{rel}}^{p}(x)+\sigma_{\mathrm{rel}}^{p}(y)=\sigma_{\mathrm{rel}}^{p}(x+y)$ & SRT3 \\
\hline$\sigma_{\mathrm{rel}}^{p}(x) \cdot y=\sigma_{\mathrm{rel}}^{p}(x \cdot y)$ & SRT4 \\
\hline$\widetilde{\widetilde{a}}+\widetilde{\widetilde{\delta}}=\widetilde{\widetilde{a}}$ & A6SRa \\
\hline$\sigma_{\text {rel }}^{r}(x)+\underset{\widetilde{\delta}}{\widetilde{\widetilde{\delta}}}=\sigma_{\text {rel }}^{r}(x)$ & $\mathrm{A} 6 \mathrm{SRb}$ \\
\hline$\widetilde{\delta} \cdot x=\widetilde{\delta}$ & A7SR \\
\hline$v_{\mathrm{rel}}^{p}(\dot{\delta})=\dot{\delta}$ & SRTO0 \\
\hline$v_{\mathrm{rel}}^{0}(x)=\dot{\delta}$ & SRTO1 \\
\hline$v_{\text {rel }}^{r}(\widetilde{\widetilde{a}})=\tilde{\widetilde{a}}$ & SRTO2 \\
\hline$v_{\mathrm{rel}}^{p+q}\left(\sigma_{\mathrm{rel}}^{p}(x)\right)=\sigma_{\mathrm{rel}}^{p}\left(v_{\mathrm{rel}}^{q}(x)\right)$ & SRTO3 \\
\hline$v_{\mathrm{rel}}^{p}(x+y)=v_{\mathrm{rel}}^{p}(x)+v_{\mathrm{rel}}^{p}(y)$ & SRTO4 \\
\hline$v_{\mathrm{rel}}^{p}(x \cdot y)=v_{\mathrm{rel}}^{p}(x) \cdot y$ & SRTO5 \\
\hline $\bar{v}_{\mathrm{rel}}^{p}(\dot{\delta})=\sigma_{\mathrm{rel}}^{p}(\dot{\delta})$ & SRI0 \\
\hline $\bar{v}_{\text {rel }}^{0}(x)=x$ & SRI1 \\
\hline $\bar{v}_{\mathrm{rel}}^{r}(\widetilde{\tilde{a}})=\sigma_{\mathrm{rel}}^{r}(\dot{\delta})$ & SRI2 \\
\hline $\bar{v}_{\mathrm{rel}}^{p+q}\left(\sigma_{\mathrm{rel}}^{p}(x)\right)=\sigma_{\mathrm{rel}}^{p}\left(\bar{v}_{\mathrm{rel}}^{q}(x)\right)$ & SRI3 \\
\hline $\bar{v}_{\mathrm{rel}}^{p}(x+y)=\bar{v}_{\mathrm{rel}}^{p}(x)+\bar{v}_{\mathrm{rel}}^{p}(y)$ & SRI4 \\
\hline $\bar{v}_{\mathrm{rel}}^{p}(x \cdot y)=\bar{v}_{\mathrm{rel}}^{p}(x) \cdot y$ & SRI5 \\
\hline
\end{tabular}

Table 15: Additional axioms for $\mathrm{BPA}^{\text {srt }}\left(a \in \mathrm{A}_{\delta}, p, q \geq 0, r>0\right)$

$(a \in \mathrm{A})$ and alternative compositions in which the form of the first operand is $\sigma_{\text {rel }}^{p}(t)$, can be eliminated in closed terms of $\mathrm{BPA}^{\text {srt }}$. The terms that remain after exhaustive elimination are called the basic terms over $\mathrm{BPA}^{\text {srt }}$. Because of this elimination result, we are permitted to use induction on the structure of basic terms over $\mathrm{BPA}^{\text {srt }}$ to prove statements for all closed terms of $\mathrm{BPA}^{\mathrm{srt}}$.

Examples We give some examples of a closed term of $\mathrm{BPA}^{\text {srt }}$ and the corresponding basic term:

$$
\begin{aligned}
& \sigma_{\text {rel }}^{5}(\widetilde{\widetilde{a}}) \cdot \sigma_{\text {rel }}^{4.9}(\widetilde{\widetilde{b}})=\sigma_{\text {rel }}^{5}\left(\widetilde{\widetilde{a}} \cdot \sigma_{\text {rel }}^{4.9}(\widetilde{\widetilde{b}})\right) \\
& \sigma_{\text {rel }}^{5}(\widetilde{\widetilde{a}}) \cdot\left(\sigma_{\text {rel }}^{4.9}(\widetilde{\widetilde{b}})+\sigma_{\text {rel }}^{5.1}(\widetilde{\widetilde{c}})\right)=\sigma_{\text {rel }}^{5}\left(\widetilde{\widetilde{a}} \cdot \sigma_{\text {rel }}^{4.9}\left(\widetilde{\widetilde{b}}+\sigma_{\text {rel }}^{0.2}(\widetilde{\widetilde{c}})\right)\right) \\
& v_{\text {rel }}^{5}\left(\sigma_{\text {rel }}^{4.9}\left((\widetilde{\widetilde{a}})+\sigma_{\text {rel }}^{5.1}(\widetilde{\widetilde{b}})\right)=\sigma_{\text {rel }}^{4.9}\left(\widetilde{\widetilde{a}}+\sigma_{\text {rel }}^{0.1}(\dot{\delta})\right)\right. \\
& \bar{v}_{\text {rel }}^{5}\left(\sigma_{\text {rel }}^{4.9}(\widetilde{\widetilde{a}})+\sigma_{\text {rel }}^{5.1}(\widetilde{\widetilde{b}})\right)=\sigma_{\text {rel }}^{5.1}(\widetilde{\widetilde{b}})
\end{aligned}
$$

Semantics of $\mathrm{BPA}^{\text {srt }}$ In case of relative timing, we can use a simple kind of real time transition system. A real time transition system with relative timing over A consists of a set of states $\mathrm{S}$, a root state $\rho \in \mathrm{S}$ and four kinds of relations on states:

a binary relation _ $\stackrel{a}{\longrightarrow}{ }_{-}$for each $a \in \mathrm{A}$, a unary relation $\_\stackrel{a}{\longrightarrow} \sqrt{ }$ for each $a \in \mathrm{A}$, a binary relation _ $\stackrel{r}{\longmapsto}$ _ for each $r \in \mathbb{R}_{>0}$, a unary relation $\mathrm{ID}\left({ }_{-}\right)$;

satisfying

1. if $s \stackrel{r+r^{\prime}}{\longmapsto} s^{\prime}, r, r^{\prime}>0$, then there is a $s^{\prime \prime}$ such that $s \stackrel{r}{\longmapsto} s^{\prime \prime}$ and $s^{\prime \prime} \stackrel{r^{\prime}}{\longmapsto} s^{\prime}$; 
2. if $s \stackrel{r}{\longmapsto} s^{\prime \prime}$ and $s^{\prime \prime} \stackrel{r^{\prime}}{\longmapsto} s^{\prime}$, then $s \stackrel{r+r^{\prime}}{\longmapsto} s^{\prime}$.

We write $\operatorname{RTTS}^{r}(A)$ for the set of all real time transition systems with relative timing over A.

We shall associate a transition system $\operatorname{TS}^{r}(t)$ in $\operatorname{RTTS}^{r}(\mathrm{~A})$ with a closed term $t$ of $\mathrm{BPA}^{\text {srt }}$ like before in the case of absolute timing. In case of relative timing, the action step, action termination, time step and immediate deadlock relations can be explained as follows:

$t \stackrel{a}{\longrightarrow} t^{\prime}$ : process $t$ is capable of first performing action $a$ without the least delay and then proceeding as process $t^{\prime}$;

$t \stackrel{a}{\longrightarrow} \sqrt{ }$ : process $t$ is capable of first performing action $a$ without the least delay and then terminating successfully;

$t \stackrel{r}{\longmapsto} t^{\prime}$ : process $t$ is capable of first idling for a time period $r$ and then proceeding as process $t^{\prime}$;

$\mathrm{ID}(t)$ : process $t$ is not capable of reaching the present time.

The structural operational semantics of $\mathrm{BPA}^{\text {srt }}$ is described by the rules given in Table 16. In one of the rules for the alternative composition operator, a negative

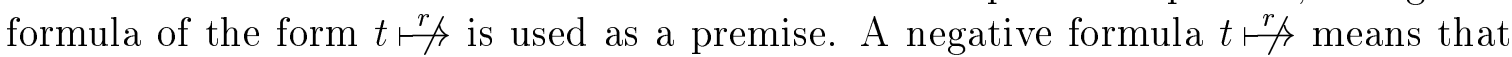
for all closed terms $t^{\prime}$ of BPA srt not $t \stackrel{r}{\longmapsto} t^{\prime}$. Hence, $t r^{r} /$ is to be read as "process $t$ is not capable of idling for a time period $r$ ".

Clearly, changing from absolute timing to relative timing leads to a significant simplification of the operational semantics. However, note that there are two rules now for the alternative composition operator concerning time related capabilities of a process $x+y$. These rules have complementary premises. Together they enforce that the choice between two idling processes is postponed till at least one of the processes cannot idle any longer.

Also the notion of bisimulation becomes simpler in case of relative timing. A bisimulation on $\operatorname{RTTS}^{r}(\mathrm{~A})$ is a symmetric binary relation $R$ on the set of states $\mathrm{S}$ such that:

1. if $R(s, t)$ and $s \stackrel{a}{\longrightarrow} s^{\prime}$, then there is a $t^{\prime}$ such that $t \stackrel{a}{\longrightarrow} t^{\prime}$ and $R\left(s^{\prime}, t^{\prime}\right)$;

2. if $R(s, t)$, then $s \stackrel{a}{\longrightarrow} \sqrt{ }$ iff $t \stackrel{a}{\longrightarrow} \sqrt{ }$;

3. if $R(s, t)$ and $s \stackrel{r}{\longmapsto} s^{\prime}$, then there is a $t^{\prime}$ such that $t \stackrel{r}{\longmapsto} t^{\prime}$ and $R\left(s^{\prime}, t^{\prime}\right)$;

4. if $R(s, t)$, then $\operatorname{ID}(s)$ iff $\operatorname{ID}(t)$.

As in the case of absolute timing, we obtain a model for $\mathrm{BPA}^{\text {srt }}$ by identifying bisimilar processes.

\subsection{Algebra of communicating processes}

In $\mathrm{ACP}^{\text {srt }}$, we have a relative counterpart of the absolute urgent initialization operator: $\nu_{\text {rel }}$ (relative urgent initialization). The process $\nu_{\text {rel }}(x)$ is the part of process $x$ that starts to perform actions without any delay. Like before in the case of absolute timing, we use the relative urgent initialization operator to axiomatize the parallel composition operator. 


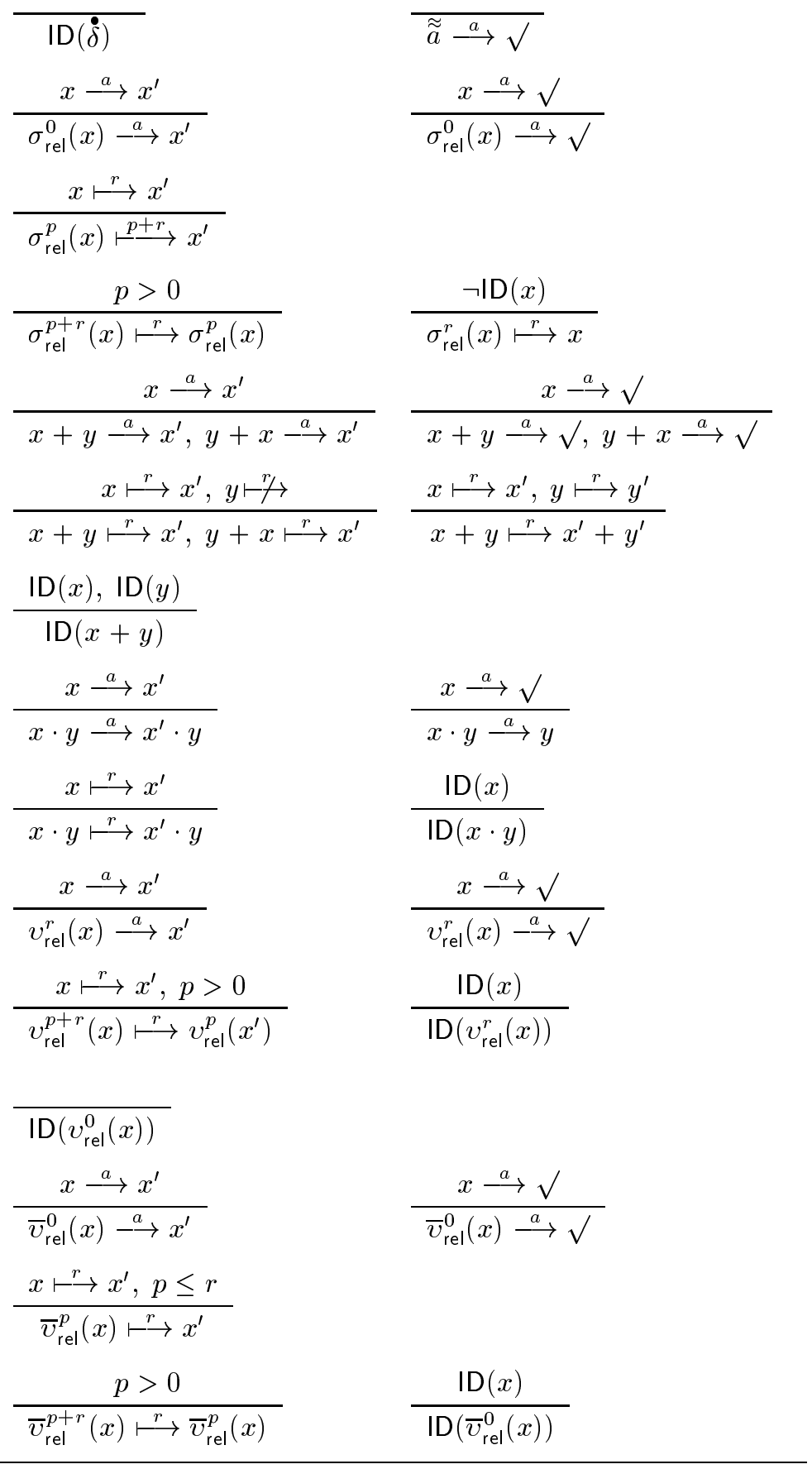

Table 16: Rules for operational semantics of $\mathrm{BPA}^{\text {srt }}(a \in \mathrm{A}, r>0, p \geq 0)$

Signature of $\mathbf{A C P}^{\text {srt }}$ The signature of $\mathrm{ACP}^{\text {srt }}$ is the signature of $\mathrm{BPA}^{\text {srt }}$ extended with the parallel composition operator $\|: \mathrm{Pr}^{\mathrm{r}} \times \mathrm{P}^{\mathrm{r}} \rightarrow \mathrm{P}^{\mathrm{r}}$, the left merge operator $\|: \mathrm{Pr}^{\mathrm{r}} \times \mathrm{Pr}^{\mathrm{r}} \rightarrow \mathrm{P}^{\mathrm{r}}$, the communication merge operator $\mid: \mathrm{P}^{\mathrm{r}} \times \mathrm{Pr}^{\mathrm{r}} \rightarrow \mathrm{Pr}^{\mathrm{r}}$, the encapsulation operators $\partial_{H}: \mathrm{Pr}^{\mathrm{r}} \rightarrow \mathrm{Pr}^{\mathrm{r}}$ (for each $H \subseteq \mathrm{A}$ ), and the relative urgent initialization operator $\nu_{\text {rel }}: \mathrm{Pr}^{\mathrm{r}} \rightarrow \mathrm{Pr}^{\mathrm{r}}$.

Axioms of $\mathrm{ACP}^{\mathrm{srt}}$ The axiom system of $\mathrm{ACP}^{\mathrm{srt}}$ consists of the axioms of $\mathrm{BPA}^{\mathrm{srt}}$ and the equations given in Table 17 . 


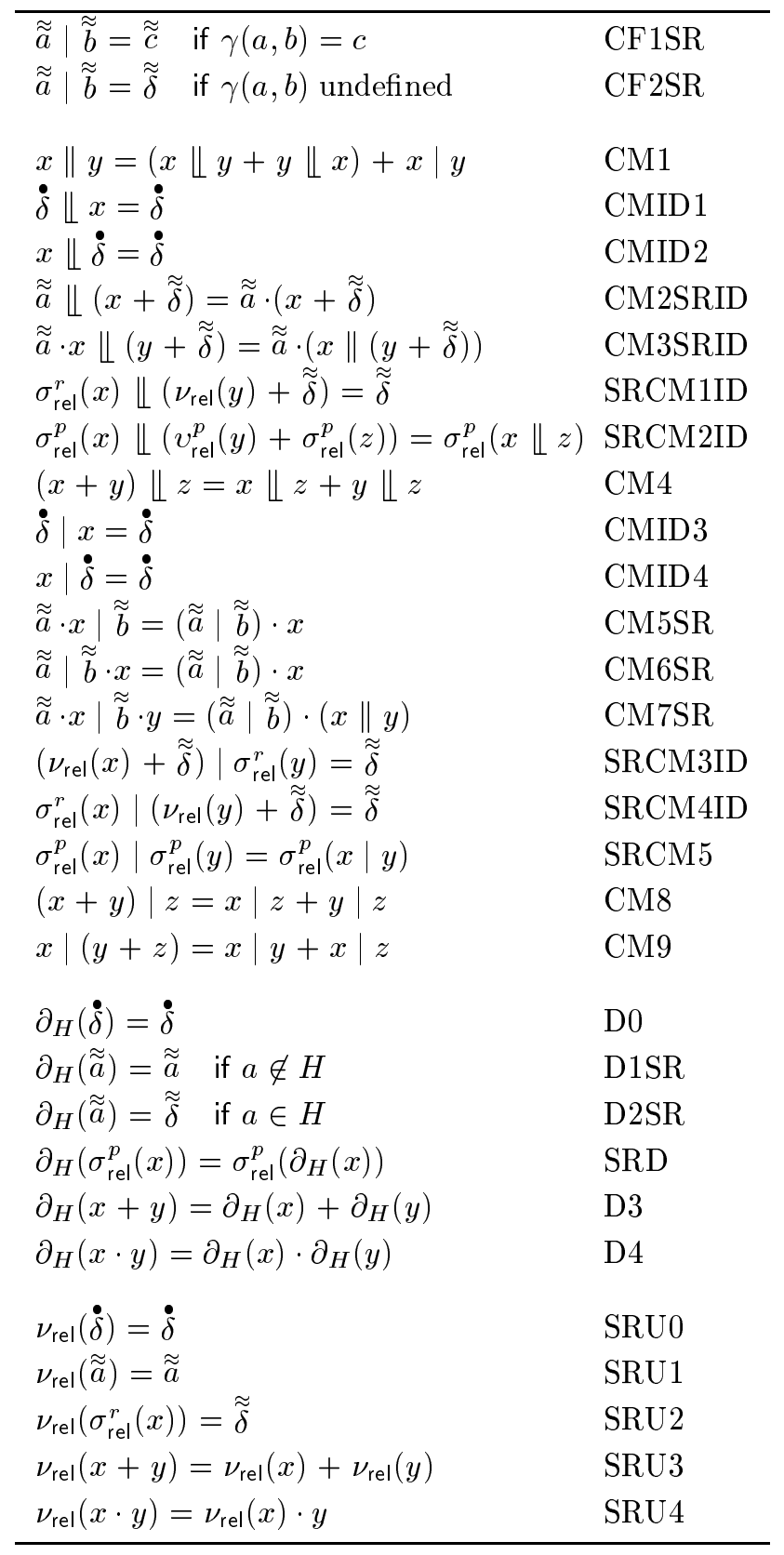

Table 17: Additional axioms for $\operatorname{ACP}^{\text {srt }}\left(a, b \in \mathrm{A}_{\delta}, c \in \mathrm{A}, p \geq 0, r>0\right)$

The additional axioms of $\mathrm{ACP}^{\mathrm{srt}}$ are just simple reformulations of the additional axioms of $\mathrm{ACP}^{\mathrm{sat}}$. That is, constants $\tilde{a}\left(a \in \mathrm{A}_{\delta}\right)$ have been replaced by constants $\tilde{\widetilde{a}}$, and the operators $\sigma_{\text {abs }}, v_{\text {abs }}$ and $\nu_{\text {abs }}$ have been replaced by $\sigma_{\text {rel }}, v_{\text {rel }}$ and $\nu_{\text {rel }}$, respectively.

Similar to the case of $\mathrm{ACP}^{\mathrm{sat}}$, we can prove that the operators $\|\|,, \mid, \partial_{H}$ and $\nu_{\text {rel }}$ can be eliminated in closed terms of $\mathrm{ACP}^{\text {srt }}$. Because of the elimination result for $\mathrm{BPA}^{\text {srt }}$, we are permitted to use induction on the structure of basic terms over BPA ${ }^{\text {srt }}$ to prove statements for all closed terms of $\mathrm{ACP}^{\mathrm{srt}}$.

Examples We give some examples of a closed term of $\mathrm{ACP}^{\text {srt }}$ and the corresponding basic term (in case $\gamma(a, c)$ is undefined): 


$$
\begin{aligned}
& \sigma_{\text {rel }}^{5}(\tilde{\widetilde{a}}) \| \sigma_{\text {rel }}^{5.1}(\widetilde{\widetilde{b}}) \cdot \sigma_{\text {rel }}^{0.3}(\widetilde{\widetilde{c}})=\sigma_{\text {rel }}^{5}\left(\widetilde{\widetilde{a}} \cdot \sigma_{\text {rel }}^{0.1}\left(\widetilde{\widetilde{b}} \cdot \sigma_{\mathrm{rel}}^{0.3}(\widetilde{\widetilde{c}})\right)\right) \\
& \sigma_{\text {rel }}^{5.1}(\widetilde{\widetilde{a}}) \| \sigma_{\text {rel }}^{5}(\widetilde{\widetilde{b}}) \cdot \sigma_{\text {rel }}^{0.3}(\widetilde{\widetilde{c}})=\sigma_{\text {rel }}^{5}\left(\widetilde{\tilde{b}} \cdot \sigma_{\text {rel }}^{0.1}\left(\widetilde{\widetilde{a}} \cdot \sigma_{\text {rel }}^{0.2}(\widetilde{\widetilde{c}})\right)\right) \\
& \sigma_{\text {rel }}^{5.1}(\widetilde{\widetilde{a}}) \| \sigma_{\text {rel }}^{4.8}(\widetilde{\vec{b}}) \cdot \sigma_{\text {rel }}^{0.3}(\widetilde{\widetilde{c}})=\sigma_{\text {rel }}^{4.8}\left(\widetilde{\tilde{b}} \cdot \sigma_{\text {rel }}^{0.3}(\widetilde{\widetilde{a}} \cdot \widetilde{\widetilde{c}}+\widetilde{\widetilde{c}} \cdot \widetilde{\widetilde{a}})\right)
\end{aligned}
$$

Semantics of $\mathrm{ACP}^{\text {srt }}$ The structural operational semantics of $\mathrm{ACP}^{\text {srt }}$ is described by the rules for $\mathrm{BPA}^{\text {srt }}$ and the rules given in Table 18 .

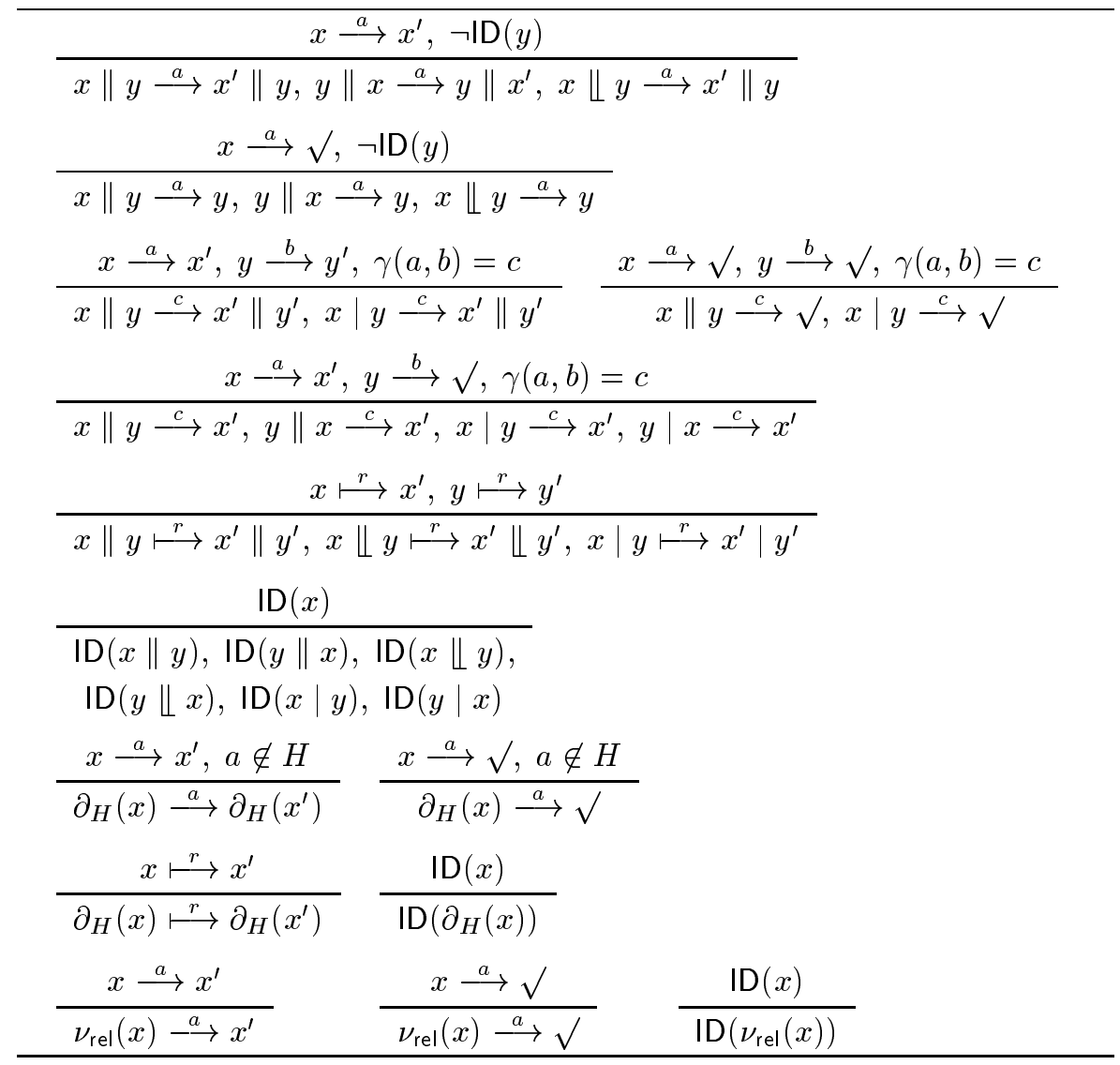

Table 18: Additional rules for $\mathrm{ACP}^{\text {srt }}(a, b, c \in \mathrm{A}, r>0)$

Changing from absolute timing to relative timing also leads to a simplification of the additional rules for parallel composition, left merge, etc. As in the previous cases, we obtain a model for $\mathrm{ACP}^{\text {srt }}$ by identifying bisimilar processes.

\subsection{Embedding $\mathrm{ACP}^{\mathrm{srt}}$ in $\mathrm{ACP}^{\mathrm{sat}} \sqrt{ }$}

Consider two theories $T$ and $T^{\prime}$. An embedding of $T$ in $T^{\prime}$ is a term structure preserving injective mapping $\epsilon$ from the terms of $T$ to the terms of $T^{\prime}$ such that for all closed terms $s, t$ of $T, s=t$ is derivable in $T$ implies $\epsilon(s)=\epsilon(t)$ is derivable in $T^{\prime}$. If there exists an embedding of $T$ in $T^{\prime}$, we say that $T$ can be embedded in $T^{\prime}$. It roughly means that what is expressible in $T$ remains expressible in $T^{\prime}$ and what is derivable in $T$ remains derivable in $T^{\prime}$. The requirement that $\epsilon$ is term structure preserving means that, for all terms $t$ of $T$ with free variables among $x_{1}, \ldots, x_{n}$ and all closed terms $t_{1}, \ldots, t_{n}$ of $T$ of appropriate sorts, $\epsilon\left(t\left[t_{1}, \ldots, t_{n} / x_{1}, \ldots, x_{n}\right]\right)=\epsilon(t)\left[\epsilon\left(t_{1}\right), \ldots, \epsilon\left(t_{n}\right) / x_{1}, \ldots, x_{n}\right]$. 
Let $f$ be an operator that is not in the signature of theory $T$. An explicit definition of $f$ in $T$ is an equation $f\left(x_{1}, \ldots, x_{n}\right)=t$ where $t$ is a term of $T$ that does not contain other free variables than $x_{1}, \ldots, x_{n}$. An extension of theory $T$ with constants and operators defined by explicit definitions in $T$ is called a definitional extension of $T$.

Consider again two theories $T$ and $T^{\prime}$. Suppose that the constants and operators in the signature of $T$ that are not in the signature of $T^{\prime}$ can be defined in $T^{\prime}$ by explicit definitions. Let $T^{\prime \prime}$ be the resulting definitional extension of $T^{\prime}$. Suppose further that the axioms of $T$ are derivable for closed terms in $T^{\prime \prime}$. Then $T$ can be embedded in $T^{\prime}$. The explicit definitions induce the following embedding:

$$
\begin{aligned}
& \epsilon(x)=x \\
& \epsilon\left(f\left(t_{1}, \ldots, t_{n}\right)\right)=f\left(\epsilon\left(t_{1}\right), \ldots, \epsilon\left(t_{n}\right)\right) \quad \text { if } f \text { in the signature of } T^{\prime} \text {; } \\
& \epsilon\left(f\left(t_{1}, \ldots, t_{n}\right)\right)=t\left[\epsilon\left(t_{1}\right), \ldots, \epsilon\left(t_{n}\right) / x_{1}, \ldots, x_{n}\right] \text { if the explicit definition of } \\
& f \text { is } f\left(x_{1}, \ldots, x_{n}\right)=t \text {. }
\end{aligned}
$$

In this chapter, we will show the existence of embeddings in the way outlined above.

The explicit definitions needed to show that $\mathrm{ACP}^{\text {srt }}$ can be embedded in $\mathrm{ACP}^{\text {sat }} \sqrt{ }$ are given in Table 19. The following lemma presents an interesting property of pro-

$$
\begin{aligned}
& \widetilde{\widetilde{a}}=\sqrt{\mathrm{s}}_{\mathrm{s}} v \cdot \sigma_{\mathrm{abs}}^{v}(\tilde{a}) \\
& \sigma_{\text {rel }}^{p}(x)=\sqrt{ }_{\mathrm{s}} v \cdot \bar{v}_{\mathrm{abs}}^{v+p}(x) \\
& v_{\mathrm{rel}}^{p}(x)=\sqrt{ }_{\mathrm{s}} v \cdot v_{\mathrm{abs}}^{v+p}\left(\bar{v}_{\mathrm{abs}}^{v}(x)\right) \\
& \bar{v}_{\mathrm{rel}}^{p}(x)=\sqrt{ }_{\mathrm{s}} v \cdot \bar{v}_{\mathrm{abs}}^{v+p}\left(\bar{v}_{\mathrm{abs}}^{v}(x)\right) \\
& \nu_{\mathrm{rel}}(x)=\sqrt{\mathrm{s}}_{\mathrm{s}} v \cdot \sigma_{\mathrm{abs}}^{v}\left(\nu_{\mathrm{abs}}(x)\right) \\
& \hline
\end{aligned}
$$

Table 19: Definitions of relative time operators $\left(a \in \mathrm{A}_{\delta}\right)$

cesses with relative timing.

Lemma 4 For each closed term $t$ of $\mathrm{ACP}^{\mathrm{sat}} \sqrt{ }$ generated by the embedded constants and operators of $\mathrm{ACP}^{\text {srt }}, \bar{v}_{\text {abs }}^{p}(t)=\sigma_{\text {abs }}^{p}(t)$.

Proof. It is straightforward to prove this by induction on the structure of $t$. We present only the case that $t$ is of the form $\widetilde{\widetilde{a}} \cdot t^{\prime}$. The other cases are similar, but simpler.

$\bar{v}_{\mathrm{abs}}^{p}\left(\tilde{\widetilde{a}} \cdot t^{\prime}\right)=\bar{v}_{\mathrm{abs}}^{p}\left(\left(\sqrt{\mathrm{s}}_{\mathrm{s}} v \cdot \sigma_{\mathrm{abs}}^{v}(\tilde{a})\right) \cdot t^{\prime}\right) \stackrel{\mathrm{SAI} 5}{=} \bar{v}_{\mathrm{abs}}^{p}\left(\left(\sqrt{\mathrm{s}}_{\mathrm{s}} v \cdot \sigma_{\mathrm{abs}}^{v}(\tilde{a})\right)\right) \cdot t^{\prime} \stackrel{\mathrm{SIA} 2}{=}$

$\bar{v}_{\mathrm{abs}}^{p}\left(\sigma_{\mathrm{abs}}^{p}(\tilde{a})\right) \cdot t^{\prime} \stackrel{\mathrm{SAI} 3^{\prime}}{=} \sigma_{\mathrm{abs}}^{p}(\tilde{a}) \cdot t^{\prime} \stackrel{\mathrm{SIA} 6}{=} \sigma_{\mathrm{abs}}^{p}(\tilde{a}) \cdot \bar{v}_{\mathrm{abs}}^{p}\left(t^{\prime}\right) \stackrel{\mathrm{IH}}{=} \sigma_{\mathrm{abs}}^{p}(\tilde{a}) \cdot \sigma_{\mathrm{abs}}^{p}\left(t^{\prime}\right) \stackrel{\mathrm{SAT} 5}{=}$

$\sigma_{\mathrm{abs}}^{p}\left(\tilde{a} \cdot \bar{v}_{\mathrm{abs}}^{0}\left(t^{\prime}\right)\right) \stackrel{\mathrm{SAT} 1,2}{=} \sigma_{\mathrm{abs}}^{p}\left(\bar{v}_{\mathrm{abs}}^{0}\left(\sigma_{\mathrm{abs}}^{0}\left(\tilde{a} \cdot \bar{v}_{\mathrm{abs}}^{0}\left(t^{\prime}\right)\right)\right) \stackrel{\text { SIA } 2}{=}\right.$

$\sigma_{\mathrm{abs}}^{p}\left(\bar{v}_{\mathrm{abs}}^{0}\left(\sqrt{\mathrm{s}}_{\mathrm{s}} v \cdot \sigma_{\mathrm{abs}}^{v}\left(\tilde{a} \cdot \bar{v}_{\mathrm{abs}}^{0}\left(t^{\prime}\right)\right)\right)\right) \stackrel{\mathrm{SAT} 1,2}{=} \sigma_{\mathrm{abs}}^{p}\left(V_{\mathrm{s}} v \cdot \sigma_{\mathrm{abs}}^{v}\left(\tilde{a} \cdot \bar{v}_{\mathrm{abs}}^{0}\left(t^{\prime}\right)\right)\right) \stackrel{\mathrm{SAT} 5, \mathrm{IH}}{=}$

$\sigma_{\mathrm{abs}}^{p}\left(\sqrt{\mathrm{s}}_{\mathrm{s}} v \cdot\left(\sigma_{\mathrm{abs}}^{v}(\tilde{a}) \cdot \bar{v}_{\mathrm{abs}}^{v}\left(t^{\prime}\right)\right)\right) \stackrel{\mathrm{SIA} 6,9}{=} \sigma_{\mathrm{abs}}^{p}\left(\left(\sqrt{\mathrm{s}} v \cdot \sigma_{\mathrm{abs}}^{v}(\tilde{a})\right) \cdot t^{\prime}\right)=\sigma_{\mathrm{abs}}^{p}\left(\tilde{\tilde{a}} \cdot t^{\prime}\right)$

Lemma 4 expresses that, for a process with relative timing, absolute initialization of the process at time $p$ is the same as shifting the process in time from time 0 to time $p$ - which implies preceding absolute initialization at time 0 . It follows from Lemma 4 and axiom SAI5 that for each pair of closed terms $t, t^{\prime}$ of $\mathrm{ACP}^{\mathrm{sat}} \sqrt{ }$ generated by the embedded constants and operators of $\mathrm{ACP}^{\mathrm{srt}}, \sigma_{\mathrm{abs}}^{p}\left(t \cdot t^{\prime}\right)=\sigma_{\mathrm{abs}}^{p}(t) \cdot t^{\prime}$. The condition that $t$ is a term generated by the embedded constants and operators of ACP ${ }^{\text {srt }}$ can not be dropped here. However, Lemma 5 points out that this condition is not a necessary one, since $\nu_{\text {abs }}(t)$ is not equal to a term generated by the embedded constants and operators of ACP srt - unless $t=\dot{\delta}$. Lemma 5 is needed in the proof of Theorem 6 . 
Lemma 5 For each pair of closed terms $t, t^{\prime}$ of $\mathrm{ACP}^{\mathrm{sat}} \sqrt{ }$ generated by the embedded constants and operators of $\mathrm{ACP}^{\mathrm{srt}}, \sigma_{\mathrm{abs}}^{p}\left(\nu_{\mathrm{abs}}(t) \cdot t^{\prime}\right)=\sigma_{\mathrm{abs}}^{p}\left(\nu_{\mathrm{abs}}(t)\right) \cdot t^{\prime}$.

Proof. It is straightforward to prove this by induction on the structure of $t$. The proof is extremely long. We present only the case that $t$ is of the form $\sigma_{\text {rel }}^{q}\left(t^{\prime \prime}\right)$. The other cases are similar, but simpler, and do not require case distinction.

Case $q=0: \sigma_{\text {abs }}^{p}\left(\nu_{\text {abs }}\left(\sigma_{\text {rel }}^{q}\left(t^{\prime \prime}\right)\right) \cdot t^{\prime}\right) \stackrel{\text { see I }}{=} \sigma_{\text {abs }}^{p}\left(\nu_{\text {abs }}\left(\sigma_{\text {abs }}^{q}\left(t^{\prime \prime}\right)\right) \cdot t^{\prime}\right) \stackrel{\text { SAT1 }}{=}$

$\sigma_{\text {abs }}^{p}\left(\nu_{\text {abs }}\left(\bar{v}_{\text {abs }}^{q}\left(t^{\prime \prime}\right)\right) \cdot t^{\prime}\right) \stackrel{\text { SII3 }}{=} \sigma_{\text {abs }}^{p}\left(\bar{v}_{\text {abs }}^{q}\left(\nu_{\text {abs }}\left(t^{\prime \prime}\right)\right) \cdot t^{\prime}\right) \stackrel{\text { SAI } 5}{=}$

$\sigma_{\text {abs }}^{p}\left(\bar{v}_{\text {abs }}^{q}\left(\nu_{\text {abs }}\left(t^{\prime \prime}\right) \cdot t^{\prime}\right)\right) \stackrel{\mathrm{SAT} 1,2}{=} \sigma_{\text {abs }}^{p}\left(\nu_{\text {abs }}\left(t^{\prime \prime}\right) \cdot t^{\prime}\right) \stackrel{\mathrm{IH}}{=} \sigma_{\mathrm{abs}}^{p}\left(\nu_{\mathrm{abs}}\left(t^{\prime \prime}\right)\right) \cdot t^{\prime} \stackrel{\mathrm{SAT} 1,2}{=}$

$\sigma_{\mathrm{abs}}^{p}\left(\bar{v}_{\mathrm{abs}}^{q}\left(\nu_{\mathrm{abs}}\left(t^{\prime \prime}\right)\right)\right) \cdot t^{\prime} \stackrel{\mathrm{SI} 13}{=} \sigma_{\mathrm{abs}}^{p}\left(\nu_{\mathrm{abs}}\left(\bar{v}_{\mathrm{abs}}^{q}\left(t^{\prime \prime}\right)\right)\right) \cdot t^{\prime} \stackrel{\mathrm{SAT} 1}{=}$

$\sigma_{\text {abs }}^{p}\left(\nu_{\text {abs }}\left(\sigma_{\text {abs }}^{q}\left(t^{\prime \prime}\right)\right)\right) \cdot t^{\prime} \stackrel{\text { see II }}{=} \sigma_{\text {abs }}^{p}\left(\nu_{\text {abs }}\left(\sigma_{\text {rel }}^{q}\left(t^{\prime \prime}\right)\right)\right) \cdot t^{\prime}$

Case $q>0: \sigma_{\text {abs }}^{p}\left(\nu_{\text {abs }}\left(\sigma_{\text {rel }}^{q}\left(t^{\prime \prime}\right)\right) \cdot t^{\prime}\right) \stackrel{\text { see I }}{=} \sigma_{\text {abs }}^{p}\left(\nu_{\text {abs }}\left(\sigma_{\text {abs }}^{q}\left(t^{\prime \prime}\right)\right) \cdot t^{\prime}\right) \stackrel{\text { SAU1 } 2}{=} \sigma_{\text {abs }}^{p}\left(\tilde{\delta} \cdot t^{\prime}\right) \stackrel{\text { SAU1 } 1}{=}$

$\sigma_{\text {abs }}^{p}\left(\nu_{\text {abs }}(\tilde{\delta}) \cdot t^{\prime}\right) \stackrel{\text { see III }}{=} \sigma_{\text {abs }}^{p}\left(\nu_{\text {abs }}(\tilde{\tilde{\delta}}) \cdot t^{\prime}\right) \stackrel{\text { IH }}{=} \sigma_{\text {abs }}^{p}\left(\nu_{\text {abs }}(\tilde{\tilde{\delta}})\right) \cdot t^{\prime} \stackrel{\text { see IV }}{=}$

$\sigma_{\text {abs }}^{p}\left(\nu_{\text {abs }}(\tilde{\delta})\right) \cdot t^{\prime} \stackrel{\text { SAU1 } 1}{=} \sigma_{\text {abs }}^{p}(\tilde{\delta}) \cdot t^{\prime} \stackrel{\text { SAU2 } 2}{=} \sigma_{\text {abs }}^{p}\left(\nu_{\text {abs }}\left(\sigma_{\text {abs }}^{q}\left(t^{\prime \prime}\right)\right)\right) \cdot t^{\prime} \stackrel{\text { see II }}{=}$

$\sigma_{\text {abs }}^{p}\left(\nu_{\text {abs }}\left(\sigma_{\text {rel }}^{q}\left(t^{\prime \prime}\right)\right)\right) \cdot t^{\prime}$

I. $\quad \sigma_{\text {abs }}^{p}\left(\nu_{\text {abs }}\left(\sigma_{\text {rel }}^{q}\left(t^{\prime \prime}\right)\right) \cdot t^{\prime}\right)=\sigma_{\text {abs }}^{p}\left(\nu_{\text {abs }}\left(\sqrt{\mathrm{s}}_{\mathrm{s}} v \cdot \bar{v}_{\mathrm{abs}}^{v+q}\left(t^{\prime \prime}\right)\right) \cdot t^{\prime}\right) \stackrel{\text { SIA16 }}{=}$

$\sigma_{\mathrm{abs}}^{p}\left(\left(\sqrt{\mathrm{s}}_{\mathrm{s}} v \cdot \nu_{\mathrm{abs}}\left(\bar{v}_{\mathrm{abs}}^{v+q}\left(t^{\prime \prime}\right)\right)\right) \cdot t^{\prime}\right) \stackrel{\text { SIA9 }}{=} \sigma_{\mathrm{abs}}^{p}\left(\sqrt{\mathrm{s}}_{\mathrm{s}} v \cdot\left(\nu_{\mathrm{abs}}\left(\bar{v}_{\mathrm{abs}}^{v+q}\left(t^{\prime \prime}\right)\right) \cdot t^{\prime}\right)\right) \stackrel{\text { SIA } 7}{=}$

$\sigma_{\text {abs }}^{p}\left(\nu_{\text {abs }}\left(\bar{v}_{\text {abs }}^{q}\left(t^{\prime \prime}\right)\right) \cdot t^{\prime}\right) \stackrel{\text { Lemma } 4}{=} \sigma_{\text {abs }}^{p}\left(\nu_{\text {abs }}\left(\sigma_{\text {abs }}^{q}\left(t^{\prime \prime}\right)\right) \cdot t^{\prime}\right)$

II, III and IV: The proofs are similar to the proof of I - axioms SAT1 and SAI1 are used in addition in III and IV.

The existence of an embedding of $\mathrm{ACP}^{\text {srt }}$ in $\mathrm{ACP}^{\mathrm{sat}} \sqrt{ }$ is established by proving the following theorem.

Theorem 6 (Embedding $\mathbf{A C P}^{\text {srt }}$ in $\mathbf{A C P}^{\text {sat }} \sqrt{ }$ ) For closed terms, the axioms of $\mathrm{ACP}^{\mathrm{srt}}$ are derivable from the axioms of $\mathrm{ACP}^{\mathrm{sat}} \sqrt{ }$ and the explicit definitions of the constants and operators $\tilde{\tilde{a}}, \sigma_{\text {rel }}, v_{\text {rel }}, \bar{v}_{\text {rel }}$, and $\nu_{\text {rel }}$ in Table 19.

Proof. The proof of this theorem is given in Appendix A.1. The proof is a matter of straightforward calculations. Equations SIAI (page 21) and DISTR $\square$ (page 22), the standard initialization axioms (Table 14, page 22), and Lemmas 4 and 5 (page 29-30) are very useful in the proof.

\section{Discrete time process algebra}

In this section, we present $\mathrm{ACP}^{\mathrm{dat}}$ and $\mathrm{ACP}^{\mathrm{drt}}$, discrete time process algebras with absolute timing and relative timing, respectively. $\mathrm{ACP}^{\mathrm{dat}}$ and $\mathrm{ACP}^{\mathrm{drt}}$ are conservative extensions of $\mathrm{ACP}_{\text {dat }}$ and $\mathrm{ACP}_{\mathrm{drt}}$ [7], respectively. First, in Section 5.1, we present $\mathrm{ACP}^{\text {dat }}$ and $\mathrm{ACP}^{\text {dat }} \sqrt{ }$, the extension of $\mathrm{ACP}^{\text {dat }}$ with initial abstraction. After that, in Section 5.2, we present ACP drt . Finally, we show in Section 5.3 how ACP dat $\sqrt{ }$ can be embedded in $\mathrm{ACP}^{\mathrm{sat}} \mathrm{I} \sqrt{ }$.

\subsection{Discrete time process algebra: absolute timing}

In this subsection, we give the signature, axioms and term model of $\mathrm{ACP}^{\text {dat }}$, a discrete time process algebra with absolute timing. $\mathrm{ACP}^{\text {dat }}$ is a conservative extension of the 
theory $\mathrm{ACP}_{\text {dat }}$, presented in [7]. Like $\mathrm{ACP}^{\text {sat }}$, it separates execution of actions and passage of time. In $\mathrm{ACP}^{\text {dat }}$, time is measured on a discrete time scale. The discrete time points divide time into time slices and timing of actions is done with respect to the time slices in which they are performed - "in time slice $n+1$ " means "at some time point $p$ such that $n \leq p<n+1$ ".

First, we treat $\mathrm{BPA}^{\text {dat }}$, basic discrete time process algebra with absolute timing, in which parallelism and communication are not considered. After that, BPA ${ }^{\text {dat }}$ is extended to $\mathrm{ACP}^{\text {dat }}$ to deal with parallelism and communication as well. Finally, initial abstraction is added.

\section{Basic process algebra}

In $\mathrm{BPA}^{\mathrm{dat}}$, we have the constants $\underline{a}$ and $\underline{\delta}$ instead of $\tilde{a}$ and $\tilde{\delta}$. The constants $\underline{a}$ and $\underline{\delta}$ stand for $a$ in time slice 1 and a deadlock in time slice 1, respectively. The operators $\sigma_{\text {abs }}, v_{\text {abs }}$ and $\bar{v}_{\text {abs }}$ have a natural number instead of a non-negative real number as their first argument. The process $\sigma_{\mathrm{abs}}^{n}(x)$ is the process $x$ shifted in time by $n$ on the discrete time scale. The process $v_{\mathrm{abs}}^{n}(x)$ is the part of $x$ that starts to perform actions before time slice $n+1$. The process $\bar{v}_{\text {abs }}^{n}(x)$ is the part of $x$ that starts to perform actions in time slice $n+1$ or a later time slice. Recall that time point $n$ is the starting-point of time slice $n+1$.

In $\mathrm{ACP}_{\text {dat }}$ [7], the notation $\mathrm{fts}(a)$ was used for actions in the first time slice. A discrete time version of ACP with absolute timing where the notation $\underline{a}$ was used earlier for actions in a time slice is $\operatorname{ACPd} \rho$ [3], but there it always carries a timestamp.

Signature of $\mathrm{BPA}^{\text {dat }}$ The signature of $\mathrm{BPA}^{\text {dat }}$ consists of the undelayable action constants $\underline{a}: \rightarrow \mathrm{P}$ (for each $a \in \mathrm{A}$ ), the undelayable deadlock constant $\underline{\delta}: \rightarrow \mathrm{P}$, the immediate deadlock constant $\dot{\delta}: \rightarrow \mathrm{P}$, the alternative composition operator + : $\mathrm{P} \times \mathrm{P} \rightarrow \mathrm{P}$, the sequential composition operator $: \mathrm{P} \times \mathrm{P} \rightarrow \mathrm{P}$, the absolute delay operator $\sigma_{\text {abs }}: \mathbb{N} \times \mathrm{P} \rightarrow \mathrm{P}$, the absolute time-out operator $v_{\text {abs }}: \mathbb{N} \times \mathrm{P} \rightarrow \mathrm{P}$, and the absolute initialization operator $\bar{v}_{\text {abs }}: \mathbb{N} \times \mathrm{P} \rightarrow \mathrm{P}$.

We denote elements of $\mathbb{N}$ by $m, m^{\prime}, n, n^{\prime}$.

Axioms of $\mathrm{BPA}^{\text {dat }}$ The axiom system of $\mathrm{BPA}^{\text {dat }}$ consists of the equations given in Tables 1 and 20 .

The axioms of $\mathrm{BPA}^{\text {dat }}$ are to a large extent simple reformulations of the axioms of $\mathrm{BPA}^{\text {sat }}$. That is, constants $\tilde{a}\left(a \in \mathrm{A}_{\delta}\right)$ have been replaced by constants $\underline{a}$, and the first argument of the operators $\sigma_{\text {abs }}, v_{\text {abs }}$ and $\bar{v}_{\text {abs }}$ has been restricted to elements of $\mathbb{N}$. Striking is the new axiom DAT7. This axiom makes the reformulations of axioms $\mathrm{A} 6 \mathrm{SAb}$ and A7SA, i.e. $\sigma_{\mathrm{abs}}^{n+1}(x)+\underline{\delta}=\sigma_{\mathrm{abs}}^{n+1}(x)$ and $\underline{\delta} \cdot x=\underline{\delta}$, derivable. Axiom DAT7 expresses that an immediate deadlock shifted in time by 1 is identified with an undelayable deadlock in the first time slice.

Like in the case of $\mathrm{BPA}^{\text {sat }}$, we can prove that the auxiliary operators $v_{\text {abs }}$ and $\bar{v}_{\text {abs }}$, as well as sequential compositions in which the form of the first operand is not $\underline{a}$ $(a \in \mathrm{A})$ and alternative compositions in which the form of the first operand is $\sigma_{\mathrm{abs}}^{n}(t)$, can be eliminated in closed terms of $\mathrm{BPA}^{\text {dat }}$. The terms that remain after exhaustive 


\begin{tabular}{ll}
\hline$\sigma_{\mathrm{abs}}^{0}(x)=\bar{v}_{\mathrm{abs}}^{0}(x)$ & DAT1 \\
$\sigma_{\mathrm{abs}}^{m}\left(\sigma_{\mathrm{abs}}^{n}(x)\right)=\sigma_{\mathrm{abs}}^{m+n}(x)$ & DAT2 \\
$\sigma_{\mathrm{abs}}^{n}(x)+\sigma_{\mathrm{abs}}^{n}(y)=\sigma_{\mathrm{abs}}^{n}(x+y)$ & DAT3 \\
$\sigma_{\mathrm{abs}}^{n}(x) \cdot v_{\mathrm{abs}}^{n}(y)=\sigma_{\mathrm{abs}}^{n}(x \cdot \dot{\delta})$ & DAT4 \\
$\sigma_{\mathrm{abs}}^{n}(x) \cdot\left(v_{\mathrm{abs}}^{n}(y)+\sigma_{\mathrm{abs}}^{n}(z)\right)=\sigma_{\mathrm{abs}}^{n}\left(x \cdot \bar{v}_{\mathrm{abs}}^{0}(z)\right)$ & DAT5 \\
$\sigma_{\mathrm{abs}}^{n}(\dot{\delta}) \cdot x=\sigma_{\mathrm{abs}}^{n}(\dot{\delta})$ & DAT6 \\
$\sigma_{\mathrm{abs}}^{1}(\dot{\delta})=\underline{\delta}$ & DAT7 \\
$\underline{a}+\underline{\delta}=\underline{a}$ & A6DAa \\
$v_{\mathrm{abs}}^{n}(\dot{\delta})=\dot{\delta}$ & DATO0 \\
$v_{\mathrm{abs}}^{0}(x)=\dot{\delta}$ & DATO1 \\
$v_{\mathrm{abs}}^{n+1}(\underline{a})=\underline{a}$ & DATO2 \\
$v_{\mathrm{abs}}^{m+n}\left(\sigma_{\mathrm{abs}}^{n}(x)\right)=\sigma_{\mathrm{abs}}^{n}\left(v_{\mathrm{abs}}^{m}(x)\right)$ & DATO3 \\
$v_{\mathrm{abs}}^{n}(x+y)=v_{\mathrm{abs}}^{n}(x)+v_{\mathrm{abs}}^{n}(y)$ & DATO4 \\
$v_{\mathrm{abs}}^{n}(x \cdot y)=v_{\mathrm{abs}}^{n}(x) \cdot y$ & DATO5 \\
$\bar{v}_{\mathrm{abs}}^{0}(\dot{\delta})=\dot{\delta}$ & \\
$\bar{v}_{\mathrm{abs}}^{n+1}(\dot{\delta})=\sigma_{\mathrm{abs}}^{n+1}(\dot{\delta})$ & DAI0a \\
$\bar{v}_{\mathrm{abs}}^{0}(\underline{a})=\underline{a}$ & DAI0b \\
$\bar{v}_{\mathrm{abs}}^{n+1}(\underline{a})=\sigma_{\mathrm{abs}}^{n+1}(\dot{\delta})$ & DAI1 \\
$\bar{v}_{\mathrm{abs}}^{m+n}\left(\sigma_{\mathrm{abs}}^{n}(x)\right)=\sigma_{\mathrm{abs}}^{n}\left(\bar{v}_{\mathrm{abs}}^{m}\left(\bar{v}_{\mathrm{abs}}^{0}(x)\right)\right)$ & DAI2 \\
$\bar{v}_{\mathrm{abs}}^{n}(x+y)=\bar{v}_{\mathrm{abs}}^{n}(x)+\bar{v}_{\mathrm{abs}}^{n}(y)$ & DAI3 \\
$\bar{v}_{\mathrm{abs}}^{n}(x \cdot y)=\bar{v}_{\mathrm{abs}}^{n}(x) \cdot y$ & DAI4 \\
\hline
\end{tabular}

Table 20: Additional axioms for $\mathrm{BPA}^{\text {dat }}\left(a \in \mathrm{A}_{\delta}\right)$

elimination are called the basic terms over BPA ${ }^{\text {dat }}$. Because of this elimination result, we are permitted to use induction on the structure of basic terms over BPA dat to prove statements for all closed terms of BPA ${ }^{\text {dat }}$.

Examples We give some examples of a closed term of $\mathrm{BPA}^{\text {dat }}$ and the corresponding basic term:

$$
\begin{aligned}
& \sigma_{\mathrm{abs}}^{1}(\underline{a}) \cdot \underline{b}=\sigma_{\mathrm{abs}}^{1}(\underline{a} \cdot \dot{\delta}) \\
& v_{\mathrm{abs}}^{3}\left(\sigma_{\mathrm{abs}}^{2}(\underline{a})+\sigma_{\mathrm{abs}}^{5}(\underline{b})\right)=\sigma_{\mathrm{abs}}^{2}(\underline{a}) \\
& \bar{v}_{\mathrm{abs}}^{3}\left(\sigma_{\mathrm{abs}}^{2}(\underline{a})+\sigma_{\mathrm{abs}}^{5}(\underline{b})\right)=\sigma_{\mathrm{abs}}^{5}(\underline{b})
\end{aligned}
$$

Semantics of BPA ${ }^{\text {dat }}$ In case a discrete time scale is used, we use a variant of real time transition systems. A discrete time transition system over A consists of a set of states $\mathrm{S}$, a root state $\rho \in \mathrm{S}$ and four kinds of relations on states:

a binary relation $\left\langle_{-}, n\right\rangle \stackrel{a}{\longrightarrow}\langle-, n\rangle$ for each $a \in \mathrm{A}, n \in \mathbb{N}$, a unary relation $\langle-, n\rangle \stackrel{a}{\longrightarrow}\langle\sqrt{ }, n\rangle$ for each $a \in \mathrm{A}, n \in \mathbb{N}$,

a binary relation $\left\langle \_, n\right\rangle \stackrel{m}{\longmapsto}\left\langle\left\langle_{-}, n^{\prime}\right\rangle\right.$ for each $m \in \mathbb{N}_{>0}, n, n^{\prime} \in \mathbb{N}$ where $n^{\prime}=n+m$, a unary relation $\operatorname{ID}\left({ }_{-}, n\right) \quad$ for each $n \in \mathbb{N}$;

satisfying

1. if $\langle s, n\rangle \stackrel{m+m^{\prime}}{\longrightarrow}\left\langle s^{\prime}, n^{\prime}\right\rangle, m, m^{\prime}>0$, then there is a $s^{\prime \prime}$ such that $\langle s, n\rangle \stackrel{m}{\longmapsto}$ $\left\langle s^{\prime \prime}, n+m\right\rangle$ and $\left\langle s^{\prime \prime}, n+m\right\rangle \stackrel{m^{\prime}}{\longmapsto}\left\langle s^{\prime}, n^{\prime}\right\rangle$; 


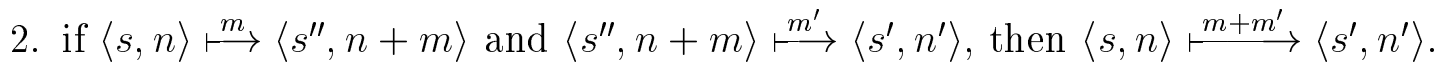

We write DTTS $(A)$ for the set of all discrete time transition systems. Associating a transition system in DTTS $(\mathrm{A})$ with a closed term $t$ of $\mathrm{BPA}^{\text {dat }}$ proceeds in essentially the same way as associating a transition system in RTTS(A) with a closed term $t$ of $\mathrm{BPA}^{\text {sat }}$. The only difference is that in the rules for the operational semantics of $\mathrm{BPA}^{\text {dat }}$ all numbers involved are restricted to $\mathbb{N}$. Therefore, we refrain from giving the rules.

Bisimulation on DTTS $(A)$ is defined as on $\operatorname{RTTS}(A)$. As in the real time cases, we obtain a model for $\mathrm{BPA}^{\text {dat }}$ by identifying bisimilar processes.

\section{Algebra of communicating processes}

In $\mathrm{ACP}^{\text {dat }}$, we do not have a discrete time counterpart of $\nu_{\mathrm{abs}}$. Unlike before in the case of real time, we can use $v_{\text {abs }}^{1}$ instead.

Signature of $\mathbf{A C P}^{\text {dat }}$ The signature of $\mathrm{ACP}^{\text {dat }}$ is the signature of $\mathrm{BPA}^{\text {dat }}$ extended with the parallel composition operator $\|: \mathrm{P} \times \mathrm{P} \rightarrow \mathrm{P}$, the left merge operator $\|$ : $\mathrm{P} \times \mathrm{P} \rightarrow \mathrm{P}$, the communication merge operator $\mathrm{I}: \mathrm{P} \times \mathrm{P} \rightarrow \mathrm{P}$, and the encapsulation operators $\partial_{H}: \mathrm{P} \rightarrow \mathrm{P}$ (for each $\left.H \subseteq \mathrm{A}\right)$.

Axioms of $\mathbf{A C P}^{\text {dat }}$ The axiom system of $\mathrm{ACP}^{\text {dat }}$ consists of the axioms of $\mathrm{BPA}^{\text {dat }}$ and the equations given in Table 21 .

The additional axioms of $\mathrm{ACP}^{\text {dat }}$ are to a large extent simple reformulations of the additional axioms of $\mathrm{ACP}^{\mathrm{sat}}$. That is, constants $\tilde{a}\left(a \in \mathrm{A}_{\delta}\right)$ have been replaced by constants $\underline{a}$, the first argument of the operators $\sigma_{\text {abs }}$ and $v_{\text {abs }}$ has been restricted to $\mathbb{N}$, and the operator $\nu_{\text {abs }}$ has been replaced by $v_{\text {abs }}^{1}$. A counterpart of SACM1 is missing. However, axiom DAT7 makes the simple reformulation of axiom SACM1, i.e. $\sigma_{\mathrm{abs}}^{n}(x) \amalg\left(v_{\mathrm{abs}}^{1}(y)+\underline{\delta}\right)=\underline{\delta}$, derivable.

As in the case of $\mathrm{ACP}^{\mathrm{sat}}$, we can prove that the operators $\|\|,, \mid$ and $\partial_{H}$ can be eliminated in closed terms of $\mathrm{ACP}^{\text {dat }}$. Because of the elimination result for $\mathrm{BPA}^{\text {dat }}$, we are permitted to use induction on the structure of basic terms over BPA dat to prove statements for all closed terms of $\mathrm{ACP}^{\text {dat }}$.

Examples We give some examples of a closed term of $\mathrm{ACP}^{\text {dat }}$ and the corresponding basic term (in case $\gamma(a, b)$ is undefined):

$$
\begin{aligned}
& \sigma_{\mathrm{abs}}^{3}(\underline{a}) \cdot\left(\sigma_{\mathrm{abs}}^{5}(\underline{b}) \| \sigma_{\mathrm{abs}}^{2}(\underline{c})\right)=\sigma_{\mathrm{abs}}^{3}(\underline{a} \cdot \dot{\delta}) \\
& \sigma_{\mathrm{abs}}^{3}(\underline{a}) \cdot \sigma_{\mathrm{abs}}^{5}(\underline{b}) \| \sigma_{\mathrm{abs}}^{2}(\underline{c})=\sigma_{\mathrm{abs}}^{2}\left(\underline{c} \cdot \sigma_{\mathrm{abs}}^{1}\left(\underline{a} \cdot \sigma_{\mathrm{abs}}^{2}(\underline{b})\right)\right) \\
& \left(\underline{a} \cdot \sigma_{\mathrm{abs}}^{2}(\underline{b})\right) \|\left(\underline{b} \cdot \sigma_{\mathrm{abs}}^{2}(\underline{a})\right)=\underline{a} \cdot\left(\underline{b} \cdot \sigma_{\mathrm{abs}}^{2}(\underline{a} \cdot \underline{b}+\underline{b} \cdot \underline{a})\right)+\underline{b} \cdot\left(\underline{a} \cdot \sigma_{\mathrm{abs}}^{2}(\underline{a} \cdot \underline{b}+\underline{b} \cdot \underline{a})\right)
\end{aligned}
$$

Semantics of $\mathrm{ACP}^{\mathrm{dat}}$ Like for the rules for the operational semantics of $\mathrm{BPA}^{\text {dat }}$, the additional rules for $\mathrm{ACP}^{\text {dat }}$ differ from the corresponding rules for the real time case only in that all numbers involved are restricted to $\mathbb{N}$. Therefore, we refrain again from giving the rules.

Again, we obtain a model for ACP dat by identifying bisimilar processes. 


\begin{tabular}{|c|c|}
\hline$\underline{a} \mid \underline{b}=\underline{c}$ if $\gamma(a, b)=c$ & CF1DA \\
\hline$\underline{a} \mid \underline{b}=\underline{\delta}$ if $\gamma(a, b)$ undefined & CF2DA \\
\hline$x \| y=(x \Downarrow y+y \llbracket x)+x \mid y$ & CM1 \\
\hline$\dot{\delta} \| x=\dot{\delta}$ & CMID1 \\
\hline$x \| \dot{\delta}=\dot{\delta}$ & CMID2 \\
\hline$\underline{a} \Perp(x+\underline{\delta})=\underline{a} \cdot(x+\underline{\delta})$ & CM2DA \\
\hline$\underline{a} \cdot x \amalg(y+\underline{\delta})=\underline{a} \cdot(x \|(y+\underline{\delta}))$ & CM3DA \\
\hline$\sigma_{\mathrm{abs}}^{n}(x) \amalg\left(v_{\mathrm{abs}}^{n}(y)+\sigma_{\mathrm{abs}}^{n}(z)\right)=\sigma_{\mathrm{abs}}^{n}(x \amalg z)$ & DACM2 \\
\hline$(x+y) \amalg z=x \Perp z+y \amalg z$ & $\mathrm{CM} 4$ \\
\hline$\dot{\delta} \mid x=\dot{\delta}$ & CMID3 \\
\hline$x \mid \dot{\delta}=\dot{\delta}$ & CMID4 \\
\hline$\underline{a} \cdot x \mid \underline{b}=(\underline{a} \mid \underline{b}) \cdot x$ & CM5DA \\
\hline$\underline{a} \mid \underline{b} \cdot x=(\underline{a} \mid \underline{b}) \cdot x$ & CM6DA \\
\hline$\underline{a} \cdot \underline{x} \mid \underline{b} \cdot y=(\underline{a} \mid \underline{b}) \cdot(x \| y)$ & CM7DA \\
\hline$\left(v_{\mathrm{abs}}^{1}(x)+\underline{\delta}\right) \mid \sigma_{\mathrm{abs}}^{n+1}(y)=\underline{\delta}$ & DACM3 \\
\hline$\sigma_{\mathrm{abs}}^{n+1}(x) \mid\left(v_{\mathrm{abs}}^{1}(y)+\underline{\delta}\right)=\underline{\delta}$ & DACM4 \\
\hline$\sigma_{\mathrm{abs}}^{n}(x) \mid \sigma_{\mathrm{abs}}^{n}(y)=\sigma_{\mathrm{abs}}^{n}(x \mid y)$ & DACM5 \\
\hline$(x+y)|z=x| z+y \mid z$ & CM8 \\
\hline$x|(y+z)=x| y+x \mid z$ & CM9 \\
\hline$\partial_{H}(\dot{\delta})=\dot{\delta}$ & D0 \\
\hline$\partial_{H}(\underline{a})=\underline{a} \quad$ if $a \notin H$ & D1DA \\
\hline$\partial_{H}(\underline{a})=\underline{\delta} \quad$ if $a \in H$ & D2DA \\
\hline$\partial_{H}\left(\sigma_{\mathrm{abs}}^{n}(x)\right)=\sigma_{\mathrm{abs}}^{n}\left(\partial_{H}(x)\right)$ & DAD \\
\hline$\partial_{H}(x+y)=\partial_{H}(x)+\partial_{H}(y)$ & D3 \\
\hline$\partial_{H}(x \cdot y)=\partial_{H}(x) \cdot \partial_{H}(y)$ & $\mathrm{D} 4$ \\
\hline
\end{tabular}

Table 21: Additional axioms for $\operatorname{ACP}^{\text {dat }}\left(a, b \in \mathrm{A}_{\delta}, c \in \mathrm{A}\right)$

\section{Initial abstraction}

We add the initial abstraction operator $\sqrt{ }_{d}$ to $\mathrm{ACP}^{\text {dat }}$. This operator is the discrete counterpart of $\sqrt{\mathrm{s}}_{\mathrm{s}}$. This means that $\sqrt{\mathrm{d}}_{i} i F$, where $i$ is a variable ranging over $\mathbb{N}$ and $F$ is a term that may contain free variables, denotes a function $f: \mathbb{N} \rightarrow \mathrm{P}$ that satisfies $f(n)=\bar{v}_{\text {abs }}^{n}(f(n))$ for all $n \in \mathbb{N}$. In the resulting theory, called $\mathrm{ACP}^{\text {dat }} \sqrt{ }$, the sort $\mathrm{P}$ of processes is replaced by the sort $\mathrm{P}^{*}$ of parametric time processes.

Signature of $\mathbf{A C P}^{\text {dat }} \sqrt{ }$ The signature of $\mathrm{ACP}^{\mathrm{dat}} \sqrt{ }$ is the signature of $\mathrm{ACP}^{\mathrm{dat}}$ extended with the initial abstraction (variable-binding) operator $\sqrt{d}_{\mathrm{d}}: \mathbb{N} . \mathrm{P}^{*} \rightarrow \mathrm{P}^{*}$.

We assume that an infinite set of variables ranging over $\mathbb{N}$ has been given, and denote them by $i, j, \ldots$. We denote terms of $\mathrm{ACP}^{\text {dat }} \sqrt{ }$ by $F, G, \ldots$.

Axiom system of $\mathbf{A C P}^{\text {dat } \sqrt{ }}$ The axiom system of $\mathrm{ACP}^{\text {dat }} \sqrt{ }$ consists of the axioms of $\mathrm{ACP}^{\text {dat }}$ and the equations given in Table 22 .

The axioms for discrete initial abstraction are simple reformulations of the axioms for standard initial abstraction. That is, the operator $\sqrt{ }_{\mathrm{s}}$ has been replaced by $\sqrt{ }_{\mathrm{d}}$, and the variables ranging over $\mathbb{R}_{\geq 0}$ have been replaced by variables ranging over $\mathbb{N}$. 


\begin{tabular}{|c|c|}
\hline$\bigvee_{d} j \cdot G=\bigvee_{d} i \cdot G[i / j]$ & DIA1 \\
\hline $\bar{v}_{\mathrm{abs}}^{n}(\sqrt{\mathrm{d}} i . F)=\bar{v}_{\mathrm{abs}}^{n}(F[n / i])$ & DIA2 \\
\hline$\bigvee_{\mathrm{d}} i \cdot\left(\bigvee_{\mathrm{d}} j \cdot F\right)=\sqrt{\mathrm{d}}_{\mathrm{d}} i \cdot F[i / j]$ & DIA3 \\
\hline$G=V_{d} i \cdot G$ & DIA4 \\
\hline$\left(\forall n \in \mathbb{N} \bullet \bar{v}_{\mathrm{abs}}^{n}(x)=\bar{v}_{\mathrm{abs}}^{n}(y)\right) \Rightarrow x=y$ & DIA5 \\
\hline$\sigma_{\mathrm{abs}}^{n}(\underline{a}) \cdot x=\sigma_{\mathrm{abs}}^{n}(\underline{a}) \cdot \bar{v}_{\mathrm{abs}}^{n}(x)$ & DIA6 \\
\hline$\sigma_{\mathrm{abs}}^{n}\left(V_{\mathrm{d}} i . F\right)=\sigma_{\mathrm{abs}}^{n}(F[0 / i])$ & DIA7 \\
\hline$(\sqrt{\mathrm{d}} i \cdot F)+G=\sqrt{\mathrm{d}}_{\mathrm{d}} i \cdot\left(F+\bar{v}_{\mathrm{abs}}^{i}(G)\right)$ & DIA8 \\
\hline$\left(V_{\mathrm{d}} i \cdot F\right) \cdot G=\bigvee_{\mathrm{d}} i \cdot(F \cdot G)$ & DIA9 \\
\hline$v_{\mathrm{abs}}^{n}(\sqrt{\mathrm{d}} i \cdot F)=\sqrt{\mathrm{d}}_{\mathrm{d}} i \cdot v_{\mathrm{abs}}^{n}(F)$ if $n \neq i$ & DIA10 \\
\hline$\left(\sqrt{d}_{\mathrm{d}} i . F\right) \| G=\sqrt{\mathrm{d}}_{\mathrm{d}} i \cdot\left(F \| \bar{v}_{\mathrm{abs}}^{i}(G)\right)$ & DIA11 \\
\hline$G \Perp\left(V_{\mathrm{d}} i . F\right)=\sqrt{\mathrm{d}}_{\mathrm{d}} i \cdot\left(\bar{v}_{\mathrm{abs}}^{i}(G) \Perp F\right)$ & DIA12 \\
\hline$(\sqrt{\mathrm{d}} i . F) \mid G=\sqrt{\mathrm{d}} i .\left(F \mid \bar{v}_{\mathrm{abs}}^{i}(G)\right)$ & DIA13 \\
\hline$G \mid\left(\sqrt{d}_{\mathrm{d}} i \cdot F\right)=\sqrt{\mathrm{d}}_{\mathrm{d}} i \cdot\left(\bar{v}_{\mathrm{abs}}^{i}(G) \mid F\right)$ & DIA 14 \\
\hline$\partial_{H}\left(\sqrt{d}_{\mathrm{d}} i . F\right)=\sqrt{\mathrm{d}} i \cdot \partial_{H}(F)$ & DIA 15 \\
\hline
\end{tabular}

Table 22: Axioms for discrete initial abstraction ( $i$ not free in $G$ )

As in the case of $\mathrm{ACP}^{\mathrm{sat}} \sqrt{ }$, all closed terms of $\mathrm{ACP}^{\mathrm{dat}} \sqrt{ }$ can be written in the form $\sqrt{\mathrm{d}} i . F$ where $F$ is a basic term over BPA ${ }^{\text {dat }}$.

Examples We give some examples of a closed term of $\mathrm{ACP}^{\text {dat }} \sqrt{ }$, the corresponding term of the form $V_{d} i$. $F$ where $F$ is a basic term and, if possible, the corresponding basic term without initial abstraction:

$$
\begin{aligned}
& \sigma_{\mathrm{abs}}^{2}\left(\sqrt{\mathrm{d}}_{\mathrm{d}} i \cdot \sigma_{\mathrm{abs}}^{i+3}(\underline{a})\right)=\sqrt{\mathrm{d}}_{\mathrm{d}} i \cdot \sigma_{\mathrm{abs}}^{5}(\underline{a})=\sigma_{\mathrm{abs}}^{5}(\underline{a}) \\
& v_{\mathrm{abs}}^{2}\left(\sqrt{\mathrm{d}}_{\mathrm{d}} i \cdot \sigma_{\mathrm{abs}}^{i+3}(\underline{a})\right)=\sqrt{\mathrm{d}}_{\mathrm{d}} i \cdot \sigma_{\mathrm{abs}}^{2}(\dot{\delta})=\sigma_{\mathrm{abs}}^{2}(\dot{\delta}) \\
& \sqrt{\mathrm{d}} i \cdot\left(V_{\mathrm{d}} j \cdot \sigma_{\mathrm{abs}}^{i+j+3}(\underline{a})\right)=\sqrt{\mathrm{d}} i \cdot \sigma_{\mathrm{abs}}^{2 i+3}(\underline{a})
\end{aligned}
$$

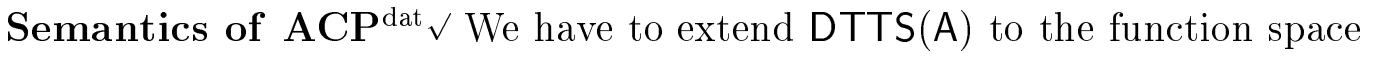

$$
\operatorname{DTTS}^{*}(\mathrm{~A})=\left\{f: \mathbb{N} \rightarrow \operatorname{DTTS}(\mathrm{A}) \mid \forall n \in \mathbb{N} \bullet f(n)=\bar{v}_{\text {abs }}^{n}(f(n))\right\}
$$

The constants and operators of $\operatorname{ACP}^{\text {dat }} \sqrt{ }$ can be defined on $\operatorname{DTTS}^{*}(A)$ in the same way as for the real time case.

We say that $f, g \in$ DTTS $^{*}$ A $)$ are bisimilar if for all $n \in \mathbb{N}$, there exists a bisimulation $R$ such that $R(f(n), g(n))$. Like before, we obtain a model of $\mathrm{ACP}^{\text {dat }} \sqrt{ }$ by defining all operators on the set of bisimulation equivalence classes.

\subsection{Discrete time process algebra: relative timing}

In this subsection, we give the signature, axioms and term model of $\mathrm{ACP}^{\mathrm{drt}}$, a discrete time process algebra with relative timing. $\mathrm{ACP}^{\mathrm{drt}}$ is a conservative extension of the theory $\mathrm{ACP}_{\mathrm{drt}}$, presented in [7]. Like $\mathrm{ACP}_{\mathrm{drt}}$, it separates execution of actions and passage of time.

First, we treat $\mathrm{BPA}^{\mathrm{drt}}$, basic discrete time process algebra with relative timing, in which parallelism and communication are not considered. After that, BPA ${ }^{\mathrm{drt}}$ is extended to $\mathrm{ACP}^{\mathrm{drt}}$ to deal with parallelism and communication as well. 


\section{Basic process algebra}

In $\mathrm{BPA}^{\mathrm{drt}}$, we have the constants $\underline{\underline{a}}$ and $\underline{\underline{\delta}}$ instead of $\underline{a}$ and $\underline{\delta}$, and the operator $\sigma_{\text {rel }}$ instead of $\sigma_{\text {abs }}$. The constants $\underline{\underline{a}}$ and $\underline{\underline{\delta}}$ stand for $a$ in the current time slice and a deadlock in the current time slice, respectively. The process $\sigma_{\text {rel }}^{n}(x)$ is the process $x$ delayed for a period of time $n$ on the discrete time scale, i.e. till the $n$-th next time slice. We have relative counterparts of the absolute time-out and initialization operators as well: $v_{\text {rel }}$ and $\bar{v}_{\text {rel }}$. The process $v_{\text {rel }}^{n}(x)$ is the part of $x$ that starts to perform actions before the $n$-th next time slice. The process $\bar{v}_{\text {rel }}^{n}(x)$ is the part of $x$ that starts to perform actions in the $n$-th next time slice or a later time slice. As in Section 4, we use $\mathrm{P}^{\mathrm{r}}$ for the sort of relative time processes.

In some presentations of $\mathrm{ACP}_{\mathrm{drt}}$, including [7], the notation cts $(a)$ was used instead of $\underline{\underline{a}}$. The notation $\underline{\underline{a}}$ for actions in the current time slice was first used in ACPdt [3].

Signature of $\mathrm{BPA}^{\mathrm{drt}}$ The signature of $\mathrm{BPA}^{\mathrm{drt}}$ consists of the undelayable action constants $\underline{\underline{a}}: \rightarrow \mathrm{Pr}^{\mathrm{r}}$ (for each $a \in \mathrm{A}$ ), the undelayable deadlock constant $\underline{\underline{\delta}}: \rightarrow \mathrm{Pr}^{\mathrm{r}}$, the immediate deadlock constant $\dot{\delta}: \rightarrow \mathrm{Pr}^{\mathrm{r}}$, the alternative composition operator + : $\mathrm{P}^{\mathrm{r}} \times \mathrm{P}^{\mathrm{r}} \rightarrow \mathrm{P}^{\mathrm{r}}$, the sequential composition operator $: \mathrm{P}^{\mathrm{r}} \times \mathrm{P}^{\mathrm{r}} \rightarrow \mathrm{P}^{\mathrm{r}}$, the relative delay operator $\sigma_{\text {rel }}: \mathbb{N} \times \mathrm{Pr}^{\mathrm{r}} \rightarrow \mathrm{P}^{\mathrm{r}}$, the relative time-out operator $v_{\text {rel }}: \mathbb{N} \times \mathrm{P}^{\mathrm{r}} \rightarrow \mathrm{P}^{\mathrm{r}}$, and the relative initialization operator $\bar{v}_{\text {rel }}: \mathbb{N} \times \mathrm{Pr}^{\mathrm{r}} \rightarrow \mathrm{Pr}^{\mathrm{r}}$.

Axioms of $\mathrm{BPA}^{\mathrm{drt}}$ The axiom system of $\mathrm{BPA}^{\mathrm{drt}}$ consists of the equations given in Tables 1 and 23 .

\begin{tabular}{|c|c|}
\hline$\sigma_{\text {rel }}^{0}(x)=x$ & DRT1 \\
\hline$\sigma_{\text {rel }}^{m}\left(\sigma_{\text {rel }}^{n}(x)\right)=\sigma_{\text {rel }}^{m+n}(x)$ & DRT2 \\
\hline$\sigma_{\mathrm{rel}}^{n}(x)+\sigma_{\mathrm{rel}}^{n}(y)=\sigma_{\mathrm{rel}}^{n}(x+y)$ & DRT3 \\
\hline$\sigma_{\mathrm{rel}}^{n}(x) \cdot y=\sigma_{\mathrm{rel}}^{n}(x \cdot y)$ & DRT4 \\
\hline$\sigma_{\text {rel }}^{1}(\dot{\delta})=\underline{\underline{\delta}}$ & DRT7 \\
\hline$\underline{\underline{a}}+\underline{\underline{\delta}}=\underline{\underline{a}}$ & A6DRa \\
\hline$v_{\mathrm{rel}}^{n}(\dot{\delta})=\dot{\delta}$ & DRTO0 \\
\hline$v_{\mathrm{rel}}^{0}(x)=\dot{\delta}$ & DRTO1 \\
\hline$v_{\mathrm{rel}}^{n+1}(\underline{\underline{a}})=\underline{\underline{a}}$ & DRTO2 \\
\hline$v_{\mathrm{rel}}^{m+n}\left(\sigma_{\mathrm{rel}}^{n}(x)\right)=\sigma_{\mathrm{rel}}^{n}\left(v_{\mathrm{rel}}^{m}(x)\right)$ & DRTO3 \\
\hline$v_{\mathrm{rel}}^{n}(x+y)=v_{\mathrm{rel}}^{n}(x)+v_{\mathrm{rel}}^{n}(y)$ & DRTO4 \\
\hline$v_{\mathrm{rel}}^{n}(x \cdot y)=v_{\mathrm{rel}}^{n}(x) \cdot y$ & DRTO5 \\
\hline $\bar{v}_{\text {rel }}^{n}(\dot{\delta})=\sigma_{\text {rel }}^{n}(\dot{\delta})$ & DRI0 \\
\hline $\bar{v}_{\mathrm{rel}}^{0}(x)=x$ & DRI1 \\
\hline $\bar{v}_{\mathrm{rel}}^{n+1}(\underline{\underline{a}})=\sigma_{\mathrm{rel}}^{n}(\underline{\underline{\delta}})$ & DRI2 \\
\hline $\bar{v}_{\mathrm{rel}}^{m+n}\left(\sigma_{\mathrm{rel}}^{n}(x)\right)=\sigma_{\mathrm{rel}}^{n}\left(\bar{v}_{\mathrm{rel}}^{m}(x)\right)$ & DRI3 \\
\hline $\bar{v}_{\mathrm{rel}}^{n}(x+y)=\bar{v}_{\mathrm{rel}}^{n}(x)+\bar{v}_{\mathrm{rel}}^{n}(y)$ & DRI4 \\
\hline $\bar{v}_{\mathrm{rel}}^{n}(x \cdot y)=\bar{v}_{\mathrm{rel}}^{n}(x) \cdot y$ & DRI5 \\
\hline
\end{tabular}

Table 23: Additional axioms for $\mathrm{BPA}^{\mathrm{drt}}\left(a \in \mathrm{A}_{\delta}\right)$

The axioms of $\mathrm{BPA}^{\mathrm{drt}}$ are to a large extent simple reformulations of the axioms of $\mathrm{BPA}^{\text {dat }}$. That is, constants $\underline{a}\left(a \in \mathrm{A}_{\delta}\right)$ have been replaced by constants $\underline{\underline{a}}$, and the 
operators $\sigma_{\text {abs }}, v_{\text {abs }}$ and $\bar{v}_{\text {abs }}$ have been replaced by $\sigma_{\text {rel }}, v_{\text {rel }}$ and $\bar{v}_{\text {rel }}$, respectively. The replacement of the axioms DAT4, DAT5 and DAT6 by the simple axiom DRT4 as well as the replacement of the axioms DAI0-DAI5 by the axioms DRI0-DRI5 are strongly reminiscent of the real time case.

Similar to the case of $\mathrm{BPA}^{\text {dat }}$, we can prove that the auxiliary operators $v_{\text {rel }}$ and $\bar{v}_{\text {rel }}$, as well as sequential compositions in which the form of the first operand is not $\underline{a}$ $(a \in \mathrm{A})$ and alternative compositions in which the form of the first operand is $\sigma_{\text {rel }}^{n}(t)$, can be eliminated in closed terms of $\mathrm{BPA}^{\mathrm{drt}}$. The terms that remain after exhaustive elimination are called the basic terms over BPA ${ }^{\mathrm{drt}}$.

Semantics of BPA ${ }^{\mathrm{drt}}$ In case of relative timing, we can use a simple kind of discrete time transition system. A discrete time transition system with relative timing over $\mathrm{A}$ consists of a set of states $\mathrm{S}$, a root state $\rho \in \mathrm{S}$ and four kinds of relations on states:

a binary relation _ $\stackrel{a}{\longrightarrow}{ }_{-}$for each $a \in \mathrm{A}$, a unary relation $\_\stackrel{a}{\longrightarrow} \sqrt{ }$ for each $a \in A$, a binary relation _ $\stackrel{n}{\longrightarrow}$ _ for each $n \in \mathbb{N}_{>0}$, a unary relation $\mathrm{ID}\left({ }_{-}\right)$;

satisfying

1. if $s \stackrel{n+n^{\prime}}{\longmapsto} s^{\prime}, n, n^{\prime}>0$, then there is a $s^{\prime \prime}$ such that $s \stackrel{n}{\longmapsto} s^{\prime \prime}$ and $s^{\prime \prime} \stackrel{n^{\prime}}{\longmapsto} s^{\prime}$;

2. if $s \stackrel{n}{\longmapsto} s^{\prime \prime}$ and $s^{\prime \prime} \stackrel{n^{\prime}}{\longmapsto} s^{\prime}$, then $s \stackrel{n+n^{\prime}}{\longmapsto} s^{\prime}$.

We write $\operatorname{DTTS}^{r}(A)$ for the set of all discrete time transition systems with relative timing over $A$. Associating a transition system in $\operatorname{DTTS}^{r}(A)$ with a closed term $t$ of BPA ${ }^{\text {drt }}$ proceeds in essentially the same way as associating a transition system in RTTS $^{r}(A)$ with a closed term $t$ of $\mathrm{BPA}^{\text {srt }}$. The only difference is that in the rules for the operational semantics of $\mathrm{BPA}^{\mathrm{drt}}$ all numbers involved are restricted to $\mathbb{N}$. Therefore, we refrain from giving the rules.

Bisimulation on $\operatorname{DTTS}^{r}(A)$ is defined as on $\operatorname{RTTS}^{r}(A)$. As in the real time cases, we obtain a model for BPA ${ }^{\text {drt }}$ by identifying bisimilar processes.

\section{Algebra of communicating processes}

Signature of $\mathbf{A C P}^{\mathrm{drt}}$ The signature of $\mathrm{ACP}^{\mathrm{drt}}$ is the signature of BPA ${ }^{\mathrm{drt}}$ extended with the parallel composition operator $\|: \mathrm{P}^{r} \times \mathrm{P}^{\mathrm{r}} \rightarrow \mathrm{P}^{\mathrm{r}}$, the left merge operator $\|$ : $\mathrm{P}^{\mathrm{r}} \times \mathrm{P}^{\mathrm{r}} \rightarrow \mathrm{P}^{\mathrm{r}}$, the communication merge operator $\mid: \mathrm{Pr}^{\mathrm{r}} \times \mathrm{P}^{\mathrm{r}} \rightarrow \mathrm{Pr}^{\mathrm{r}}$, and the encapsulation operators $\partial_{H}: \mathrm{P}^{\mathrm{r}} \rightarrow \mathrm{P}^{\mathrm{r}}$ (for each $\left.H \subseteq \mathrm{A}\right)$.

Axioms of $\mathrm{ACP}^{\mathrm{drt}}$ The axiom system of $\mathrm{ACP}^{\mathrm{drt}}$ consists of the axioms of $\mathrm{BPA}^{\mathrm{drt}}$ and the equations given in Table 24 .

The additional axioms of $\mathrm{ACP}^{\mathrm{drt}}$ are just simple reformulations of the additional axioms of $\mathrm{ACP}^{\mathrm{dat}}$. That is, constants $\underline{a}\left(a \in \mathrm{A}_{\delta}\right)$ have been replaced by constants $\underline{\underline{a}}$, and the operators $\sigma_{\text {abs }}$ and $v_{\text {abs }}$ have been replaced by $\sigma_{\text {rel }}$ and $v_{\text {rel }}$, respectively.

As in the case of $A C P^{\text {dat }}$, we can prove that the operators $\|\|,, \mid$ and $\partial_{H}$ can be eliminated in closed terms of $\mathrm{ACP}^{\mathrm{drt}}$. 


\begin{tabular}{|c|c|}
\hline $\begin{array}{ll}\underline{\underline{a}} \mid \underline{\underline{b}}=\underline{\underline{c}} & \text { if } \gamma(a, b)=c \\
\underline{\underline{a}} \mid \underline{\underline{b}}=\underline{\underline{\delta}} & \text { if } \gamma(a, b) \text { undefined }\end{array}$ & $\begin{array}{l}\text { CF1DR } \\
\text { CF2DR }\end{array}$ \\
\hline$x \| y=(x \| y+y \llbracket x)+x \mid y$ & CM1 \\
\hline$\dot{\delta} \| x=\dot{\delta}$ & CMID1 \\
\hline$x \| \dot{\delta}=\dot{\delta}$ & CMID2 \\
\hline$\underline{\underline{a}} \Perp(x+\underline{\underline{\delta}})=\underline{\underline{a}} \cdot(x+\underline{\underline{\delta}})$ & CM2DRID \\
\hline$\underline{\underline{a}} \cdot x \|(y+\underline{\underline{\delta}})=\underline{\underline{a}} \cdot(x \|(y+\underline{\underline{\delta}}))$ & CM3DRID \\
\hline $\bar{\sigma}_{\mathrm{rel}}^{n+1}(x) \Downarrow\left(\bar{v}_{\mathrm{rel}}^{n+1}(y)+\sigma_{\mathrm{rel}}^{n+1}(z)\right)=\sigma_{\mathrm{rel}}^{n+1}(x \Perp z)$ & DRCM2 \\
\hline$(x+y) \llbracket z=x \Perp z+y \amalg z$ & CM4 \\
\hline$\dot{\delta} \mid x=\dot{\delta}$ & CMID3 \\
\hline$x \mid \dot{\delta}=\dot{\delta}$ & CMID4 \\
\hline$\underline{\underline{a}} \cdot x \mid \underline{\underline{b}}=(\underline{\underline{a}} \mid \underline{\underline{b}}) \cdot x$ & CM5DR \\
\hline$\overline{\underline{a}} \mid \underline{\underline{b}} \cdot \bar{x}=(\overline{\underline{a}} \mid \underline{\underline{b}}) \cdot x$ & CM6DR \\
\hline$\underline{\underline{a}} \cdot \bar{x} \mid \underline{\underline{b}} \cdot y=(\overline{\underline{a}} \mid \underline{\underline{b}}) \cdot(x \| y)$ & CM7DR \\
\hline$\left.\overline{\left(v_{\mathrm{rel}}^{1}(x)\right.}+\underline{\underline{\delta}}\right) \mid \bar{\sigma}_{\mathrm{rel}}^{n \overline{+}}(y)=\underline{\underline{\delta}}$ & DRCM3ID \\
\hline$\sigma_{\mathrm{rel}}^{n+1}(x) \mid\left(v_{\mathrm{rel}}^{1}(y)+\underline{\delta}\right)=\underline{\bar{\delta}}$ & DRCM4ID \\
\hline$\sigma_{\text {rel }}^{n+1}(x) \mid \sigma_{\text {rel }}^{n+1}(y)=\sigma_{\text {rel }}^{n+1}(x \mid y)$ & DRCM5 \\
\hline$(x+y)|z=x| z+y \mid z$ & CM8 \\
\hline$x|(y+z)=x| y+x \mid z$ & CM9 \\
\hline$\partial_{H}(\dot{\delta})=\dot{\delta}$ & D0 \\
\hline$\partial_{H}(\underline{\underline{a}})=\underline{\underline{a}}$ if $a \notin H$ & D1DR \\
\hline$\partial_{H}(\underline{\underline{\bar{a}}})=\overline{\overline{\underline{\delta}}} \quad$ if $a \in H$ & D2DR \\
\hline$\partial_{H}\left(\bar{\sigma}_{\text {rel }}^{n}(x)\right)=\sigma_{\text {rel }}^{n}\left(\partial_{H}(x)\right)$ & $\mathrm{DRD}$ \\
\hline$\partial_{H}(x+y)=\partial_{H}(x)+\partial_{H}(y)$ & D3 \\
\hline$\partial_{H}(x \cdot y)=\partial_{H}(x) \cdot \partial_{H}(y)$ & $\mathrm{D} 4$ \\
\hline
\end{tabular}

Table 24: Additional axioms for $\operatorname{ACP}^{\mathrm{drt}}\left(a, b \in \mathrm{A}_{\delta}, c \in \mathrm{A}\right)$

Semantics of $\mathbf{A C P}^{\mathrm{drt}}$ Like for the rules for the operational semantics of $\mathrm{BPA}^{\mathrm{drt}}$, the additional rules for $\mathrm{ACP}^{\mathrm{drt}}$ differ from the corresponding rules for the real time case only in that all numbers involved are restricted to $\mathbb{N}$. Therefore, we refrain again from giving the rules.

Again, we obtain a model for $\mathrm{ACP}^{\mathrm{drt}}$ by identifying bisimilar processes.

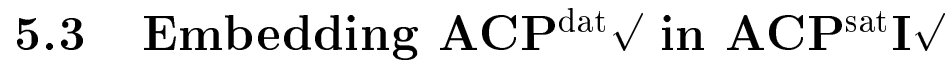

In this subsection, we will show that $\mathrm{ACP}^{\text {dat }} \sqrt{ }$ can be embedded in $\mathrm{ACP}^{\mathrm{sat}} \mathrm{I} \sqrt{ }$. We will do so in the way outlined in Section 4.3. The explicit definitions needed are given in Table 25. Notice that the operators $\sigma_{\text {abs }}, v_{\text {abs }}$ and $\bar{v}_{\text {abs }}$ of ACP $\mathrm{Adat} \sqrt{ }$ are simply defined as the operators $\sigma_{\text {abs }}, v_{\text {abs }}$ and $\bar{v}_{\text {abs }}$ of ACPsat $\mathrm{I} \checkmark$ restricted in their first argument to $\mathbb{N}$. We will establish the existence of an embedding by proving that for closed terms the axioms of $\mathrm{ACP}^{\mathrm{dat}} \sqrt{ }$ are derivable from the axioms of $\mathrm{ACP}^{\mathrm{sat}} \mathrm{I} \checkmark$ and the explicit definitions given in Table 25. However, we first take another look at the connection between $\mathrm{ACP}^{\mathrm{sat}} \mathrm{I} \sqrt{ }$ and $\mathrm{ACP} \mathrm{P}^{\text {dat }} \sqrt{ }$ by introducing the notions of a discretized real time process and a discretely initialized real time process. 


\begin{tabular}{l}
$\underline{a}=\int_{v \in[0,1)} \sigma_{\mathrm{abs}}^{v}(\tilde{a})$ \\
$\sigma_{\mathrm{abs}}^{n}(x)=\sigma_{\mathrm{abs}}^{n}(x)$ \\
$v_{\mathrm{abs}}^{n}(x)=v_{\mathrm{abs}}^{n}(x)$ \\
$\bar{v}_{\mathrm{abs}}^{n}(x)=\bar{v}_{\mathrm{abs}}^{n}(x)$ \\
$V_{\mathrm{d}} i \cdot F=\sqrt{\mathrm{s}} v \cdot F[\lfloor v\rfloor / i]$ \\
\hline
\end{tabular}

Table 25: Definitions of discrete time operators $\left(a \in \mathrm{A}_{\delta}\right)$

In Section 3.2, we have introduced the model $\mathrm{M}_{\mathrm{A}}^{*}$ of $\mathrm{ACP}^{\mathrm{sat}} \mathrm{I} \sqrt{ }$. The model of $\mathrm{ACP}^{\text {dat }} \sqrt{ }$ outlined in Section 5.1 is isomorphic to the subalgebra of $\mathrm{M}_{\mathrm{A}}^{*}$ generated by the embedded constants and operators of ACPdat $\sqrt{ }$. The domain of this subalgebra consists of those real time processes, i.e. elements of the domain of $M_{A}^{*}$, that are discretized. We define the notion of a discretized real time process in terms of the auxiliary discretization operator $\mathcal{D}: \mathrm{P}^{*} \rightarrow \mathrm{P}^{*}$ of which the defining axioms are given in Table 26. A real time process $x$ is a discretized real time process, written

$$
\begin{aligned}
& \mathcal{D}(\dot{\delta})=\dot{\delta} \\
& \mathcal{D}(\tilde{a})=\underline{a} \\
& \mathcal{D}\left(\sigma_{\mathrm{abs}}^{p}(x)\right)=\sigma_{\mathrm{abs}}^{\lfloor p\rfloor}(\mathcal{D}(x)) \\
& \mathcal{D}(x+y)=\mathcal{D}(x)+\mathcal{D}(y) \\
& \mathcal{D}(x \cdot y)=\mathcal{D}(x) \cdot \mathcal{D}(y) \\
& \mathcal{D}\left(\int_{v \in V} F\right)=\int_{v \in V} \mathcal{D}(F) \\
& \mathcal{D}\left(\sqrt{s}_{\mathrm{s}} v \cdot F\right)=\sqrt{\mathrm{s}}_{\mathrm{s}} v \cdot \mathcal{D}(F) \\
& \hline
\end{aligned}
$$

Table 26: Definition of discretization $\left(a \in \mathrm{A}_{\delta}\right)$

$x \in$ DIS, if $x=\mathcal{D}(x)$. The properties given in Table 27 express that the set of all discretized real time processes is closed under the operators of $\mathrm{ACP}^{\text {dat }} \sqrt{ }$, integration and discretization.

$$
\begin{aligned}
& \dot{\dot{\delta}}, \underline{a} \in \mathrm{DIS} \\
& x \in \mathrm{DIS} \Rightarrow \sigma_{\mathrm{abs}}^{n}(x), v_{\mathrm{abs}}^{n}(x), \bar{v}_{\mathrm{abs}}^{n}(x), \partial_{H}(x) \in \mathrm{DIS} \\
& x, y \in \mathrm{DIS} \Rightarrow x+y, x \cdot y, x\|y, x\| y, x \mid y \in \mathrm{DIS} \\
& (\forall n \in \mathbb{N} \bullet F[n / i] \in \mathrm{DIS}) \Rightarrow \sqrt{\mathrm{d}}_{\mathrm{d}} i \cdot F \in \mathrm{DIS} \\
& (\forall p \in V \cdot F[p / v] \in \mathrm{DIS}) \Rightarrow \int_{v \in V} F \in \mathrm{DIS} \\
& x \in \mathrm{DIS} \Rightarrow \mathcal{D}(x) \in \mathrm{DIS}
\end{aligned}
$$

Table 27: Properties of discretized processes $\left(a \in \mathrm{A}_{\delta}\right)$

For elements $f$ of $\operatorname{RTTS}^{*}(\mathrm{~A})$, the discretization of $f, \mathcal{D}(f)$, is obtained as follows $\left(t \in \mathbb{R}_{\geq 0}, q=p+r\right.$ and $\left.q^{\prime}=p+r^{\prime}\right)$ :

1. for each $t$, if $\langle s, p\rangle \stackrel{a}{\longrightarrow}\left\langle s^{\prime}, p\right\rangle$ in $f(t)$, then $\left\langle s, p^{\prime}\right\rangle \stackrel{a}{\longrightarrow}\left\langle s^{\prime}, p^{\prime}\right\rangle$ in $\mathcal{D}(f)(t)$ for each $p^{\prime} \in[\lfloor p\rfloor,\lfloor p+1\rfloor)$

2. for each $t$, if $\langle s, p\rangle \stackrel{a}{\longrightarrow}\langle\sqrt{ }, p\rangle$ in $f(t)$, then $\left\langle s, p^{\prime}\right\rangle \stackrel{a}{\longrightarrow}\left\langle\sqrt{ }, p^{\prime}\right\rangle$ in $\mathcal{D}(f)(t)$ for each $p^{\prime} \in[\lfloor p\rfloor,\lfloor p+1\rfloor)$

3. for each $t$, if $\langle s, p\rangle \stackrel{r}{\longmapsto}\langle s, q\rangle$ in $f(t)$, then $\langle s, p\rangle \stackrel{r^{\prime}}{\longmapsto}\left\langle s, q^{\prime}\right\rangle$ in $\mathcal{D}(f)(t)$ for each $q^{\prime} \in[q,\lfloor q+1\rfloor)$ 
4. for each $t$, if $\operatorname{ID}(s, p)$ in $f(t)$, then $\operatorname{ID}(s, p)$ in $\mathcal{D}(f)(t)$;

5 . for each $t$, if neither $\operatorname{ID}(s, p)$ in $f(t)$ nor either $\langle s, p\rangle \stackrel{a}{\longrightarrow}\left\langle s^{\prime}, p\right\rangle$ in $f(t)$ for some $a, s^{\prime}$ or $\langle s, p\rangle \stackrel{r}{\longmapsto}\langle s, q\rangle$ in $f(t)$ for some $r$, then $\langle s, p\rangle \stackrel{r^{\prime}}{\longmapsto}\left\langle s, q^{\prime}\right\rangle$ in $\mathcal{D}(f)(t)$ for each $q^{\prime} \in(p,\lfloor p+1\rfloor)$.

Hence, for real time processes corresponding to discrete time processes, the following holds: if an action can be performed at some time $p$ such that $n \leq p<n+1$, it can also be performed at any other time $p^{\prime}$ such that $n \leq p^{\prime}<n+1$.

A real time process $x$ is a discretely initialized real time process, written $x \in$ DIP, if $x=\sqrt{\mathrm{d}}_{\mathrm{d}} i \cdot \bar{v}_{\mathrm{abs}}^{i}(x)$. It follows immediately that $x \in \mathrm{DIP} \Leftrightarrow x=\sqrt{\mathrm{s}}_{\mathrm{s}} v \cdot \bar{v}_{\mathrm{abs}}^{\lfloor v\rfloor}(x)$. It is easy to show by induction on the term structure that all discretized processes are discretely initialized, i.e. $x \in$ DIS $\Rightarrow x \in$ DIP. Not all discretely initialized processes are discretized, e.g. $\sqrt{\mathrm{s}}_{\mathrm{s}} v \cdot \sigma_{\mathrm{abs}}^{\lfloor v+1\rfloor}(\tilde{a}) \in \mathrm{DIP}$ and $\sqrt{\mathrm{s}}_{\mathrm{s}} v \cdot \sigma_{\mathrm{abs}}^{\lfloor v+1\rfloor}(\tilde{a}) \notin \mathrm{DIS}$. This means that for real time processes corresponding to discrete time processes, initialization takes always place at discrete points in time; and that there are real time processes not corresponding to discrete time processes for which initialization takes always place at discrete points in time.

Lemma 7 For each closed term $t$ of ACP ${ }^{s a t} \mathrm{I} \vee$ generated by the embedded constants and operators of $\mathrm{ACP}^{\mathrm{dat}} \sqrt{ }, t=\sqrt{\mathrm{s}}_{\mathrm{s}} v \cdot \bar{v}_{\mathrm{abs}}^{\lfloor v\rfloor}(t)$.

Proof. From the properties given in Table 27, we know that each process $x$ generated by the embedded constants and operators of ACPdat $\sqrt{ }$ is discretized, i.e. $x \in$ DIS. Because $x \in \mathrm{DIS} \Rightarrow x \in \mathrm{DIP}$ and $x \in \mathrm{DIP} \Leftrightarrow x=\sqrt{\mathrm{s}} v \cdot \bar{v}_{\text {abs }}^{\lfloor v\rfloor}(x)$, the result immediately follows.

The following lemmas present other useful properties of discrete time processes.

Lemma 8 For each closed term $t$ of $\mathrm{ACP}^{\mathrm{sat}} \mathrm{I} \checkmark$ generated by the embedded constants and operators of $\mathrm{ACP}^{\mathrm{dat}} \sqrt{ }$, there exists a term $t^{\prime}$ containing no other free variable than $v$ such that for each $p \in \mathbb{R}_{\geq 0}: \bar{v}_{\text {abs }}^{p}(t)=\sigma_{\text {abs }}^{p}\left(t^{\prime}[p / v]\right), t^{\prime}[p / v]=\bar{v}_{\text {abs }}^{0}\left(t^{\prime}[p / v]\right)$, and if $p \in[0,1)$ and $t \neq \dot{\delta}, t^{\prime}[p / v]=t^{\prime}[p / v]+\sigma_{\text {abs }}^{1-p}(\delta)$ and $\bar{v}_{\text {abs }}^{p}(t+\underline{\delta})=\sigma_{\text {abs }}^{p}\left(t^{\prime}[p / v]+\tilde{\delta}\right)$. In subsequent proofs, we write $t_{[v]}$ for a fixed but arbitrary term $t^{\prime}$ that fulfills these conditions.

Proof. Observe that, if $p \in[0,1)$ and $t \neq \dot{\delta}, \bar{v}_{\text {abs }}^{p}(t+\underline{\delta})=\sigma_{\text {abs }}^{p}\left(t^{\prime}[p / v]+\tilde{\delta}\right)$ follows directly from $\bar{v}_{\text {abs }}^{p}(t)=\sigma_{\text {abs }}^{p}\left(t^{\prime}[p / v]\right)$ and $t^{\prime}[p / v]=t^{\prime}[p / v]+\sigma_{\text {abs }}^{1-p}(\dot{\delta})$. Observe further that, if $t^{\prime}$ is a term that fulfills all above-mentioned conditions but $t^{\prime}[p / v]=\bar{v}_{\text {abs }}^{0}\left(t^{\prime}[p / v]\right), \bar{v}_{\text {abs }}^{0}\left(t^{\prime}\right)$ is a term that fulfills all conditions. Consequently, it suffices to prove that there exists a $t^{\prime}$ such that $\bar{v}_{\text {abs }}^{p}(t)=\sigma_{\text {abs }}^{p}\left(t^{\prime}[p / v]\right)$ and, if $p \in[0,1)$ and $t \neq \dot{\delta}, t^{\prime}[p / v]=t^{\prime}[p / v]+\sigma_{\text {abs }}^{1-p}(\dot{\delta})$. It is straightforward to prove this by induction on the structure of $t$. We present only the case that $t$ is of the form $\underline{a}$. The other cases are simpler or similar to corresponding cases in the proof of Lemma 3 . 


$$
\begin{aligned}
& \bar{v}_{\mathrm{abs}}^{p}(\underline{a})=\bar{v}_{\mathrm{abs}}^{p}\left(\int_{w \in[0,1)} \sigma_{\mathrm{abs}}^{w}(\tilde{a})\right) \stackrel{\mathrm{SAI} 6}{=} \int_{w \in[0,1)} \bar{v}_{\mathrm{abs}}^{p}\left(\sigma_{\mathrm{abs}}^{w}(\tilde{a})\right) \stackrel{\mathrm{INT} 4}{=}
\end{aligned}
$$

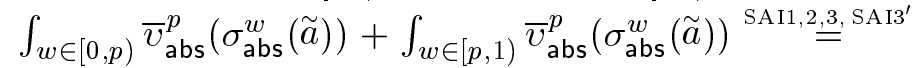

$$
\begin{aligned}
& \int_{w \in[0, p)} \sigma_{\mathrm{abs}}^{p}(\dot{\delta})+\int_{w \in[p, 1)} \sigma_{\mathrm{abs}}^{w}(\tilde{a}) \stackrel{\text { INT5 }}{=} \sigma_{\mathrm{abs}}^{p}(\dot{\delta})+\int_{w \in[p, 1)} \sigma_{\mathrm{abs}}^{w}(\tilde{a}) \stackrel{\text { INT5 }}{=} \\
& \int_{w \in[p, 1)} \sigma_{\mathrm{abs}}^{p}(\dot{\delta})+\int_{w \in[p, 1)} \sigma_{\mathrm{abs}}^{w}(\tilde{a}) \stackrel{\text { INT11 }}{=} \int_{w \in[p, 1)}\left(\sigma_{\mathrm{abs}}^{p}(\dot{\delta})+\sigma_{\mathrm{abs}}^{w}(\tilde{a})\right) \stackrel{\mathrm{SAT} 2}{=} \\
& \int_{w \in[p, 1)}\left(\sigma_{\text {abs }}^{p}(\dot{\delta})+\sigma_{\text {abs }}^{p}\left(\sigma_{\text {abs }}^{w-p}(\tilde{a})\right)\right) \stackrel{\text { SAT3 }}{=} \int_{w \in[p, 1)} \sigma_{\text {abs }}^{p}\left(\dot{\delta}+\sigma_{\text {abs }}^{w-p}(\tilde{a})\right) \stackrel{\text { A6ID }}{=} \\
& \int_{w \in[p, 1)} \sigma_{\mathrm{abs}}^{p}\left(\sigma_{\mathrm{abs}}^{w-p}(\tilde{a})\right) \stackrel{\mathrm{INT} 10}{=} \sigma_{\mathrm{abs}}^{p}\left(\int_{w \in[p, 1)} \sigma_{\mathrm{abs}}^{w-p}(\tilde{a})\right)=\sigma_{\mathrm{abs}}^{p}\left(\int_{w \in[0,1-p)} \sigma_{\mathrm{abs}}^{w}(\tilde{a})\right)= \\
& \sigma_{\mathrm{abs}}^{p}\left(\left(\int_{w \in[0,1-v)} \sigma_{\mathrm{abs}}^{w}(\tilde{a})\right)[p / v]\right) \text { and } \\
& \int_{w \in[0,1-p)} \sigma_{\mathrm{abs}}^{w}(\tilde{a}) \stackrel{\mathrm{A} 6 \mathrm{SAa}}{=} \int_{w \in[0,1-p)} \sigma_{\mathrm{abs}}^{w}(\tilde{a}+\tilde{\delta}) \stackrel{\mathrm{SAT} 3}{=} \int_{w \in[0,1-p)}\left(\sigma_{\mathrm{abs}}^{w}(\tilde{a})+\sigma_{\mathrm{abs}}^{w}(\tilde{\delta})\right) \stackrel{\text { INT11 }}{=} \\
& \int_{w \in[0,1-p)} \sigma_{\mathrm{abs}}^{w}(\tilde{a})+\int_{w \in[0,1-p)} \sigma_{\mathrm{abs}}^{w}(\tilde{\delta}) \stackrel{\mathrm{INT} 8}{=} \int_{w \in[0,1-p)} \sigma_{\mathrm{abs}}^{w}(\tilde{a})+\sigma_{\mathrm{abs}}^{1-p}(\dot{\delta})
\end{aligned}
$$

Lemma 9 For each $p \in \mathbb{R}_{>0}$ and closed term $t$ of $\mathrm{ACP}^{\mathrm{sat}} \mathrm{I} \sqrt{ }$ generated by the embedded constants and operators of $\mathrm{ACP}^{\mathrm{dat}} \sqrt{ }$, there exists a closed term $t^{\prime}$ such that $\bar{v}_{\mathrm{abs}}^{p}(t)=$ $\sigma_{\mathrm{abs}}^{p}\left(t^{\prime}\right), t^{\prime}=\bar{v}_{\mathrm{abs}}^{0}\left(t^{\prime}\right)$, and if $p \in[0,1)$ and $t \neq \dot{\delta}, t^{\prime}=t^{\prime}+\sigma_{\mathrm{abs}}^{1-p}(\dot{\delta})$ and $\bar{v}_{\mathrm{abs}}^{p}(t+\underline{\delta})=$ $\sigma_{\text {abs }}^{p}\left(t^{\prime}+\tilde{\delta}\right)$. In subsequent proofs, we write $t_{[p]}$ for a fixed but arbitrary closed term $t^{\prime}$ that fulfills these conditions - like in case of applications of Lemma 3.

Proof. This follows immediately from Lemma 8.

Lemma 10 For each closed term $t$ of $\mathrm{ACP}^{\mathrm{sat}} \mathrm{I} \checkmark$ generated by the embedded constants and operators of $\mathrm{ACP}^{\mathrm{dat}} \sqrt{ }$, there exists a closed term $t^{\prime}$ such that $v_{\mathrm{abs}}^{1}(t+\underline{\delta})=$ $\int_{v \in[0,1)} \sigma_{\mathrm{abs}}^{v}\left(\nu_{\mathrm{abs}}\left(t^{\prime}\right)+\tilde{\delta}\right)$. In subsequent proofs we write $t^{\circ}$ for a fixed but arbitrary closed term $t^{\prime}$ that fulfills this condition.

Proof. It is straightforward to prove this by induction on the structure of $t$. We present only the case that $t$ is of the form $\underline{a} \cdot t^{\prime \prime}$. The other cases are simpler.

$$
\begin{aligned}
& v_{\mathrm{abs}}^{1}\left(\underline{a} \cdot t^{\prime \prime}+\underline{\delta}\right)=v_{\mathrm{abs}}^{1}\left(\left(\int_{v \in[0,1)} \sigma_{\mathrm{abs}}^{v}(\tilde{a})\right) \cdot t^{\prime \prime}+\int_{v \in[0,1)} \sigma_{\mathrm{abs}}^{v}(\tilde{\delta})\right) \stackrel{\text { SATO } 4,5}{=} \\
& v_{\mathrm{abs}}^{1}\left(\int_{v \in[0,1)} \sigma_{\mathrm{abs}}^{v}(\tilde{a})\right) \cdot t^{\prime \prime}+v_{\mathrm{abs}}^{1}\left(\int_{v \in[0,1)} \sigma_{\mathrm{abs}}^{v}(\tilde{\delta})\right) \stackrel{\mathrm{SATO} 2,3,6}{=} \\
& \left(\int_{v \in[0,1)} \sigma_{\mathrm{abs}}^{v}(\tilde{a})\right) \cdot t^{\prime \prime}+\int_{v \in[0,1)} \sigma_{\mathrm{abs}}^{v}(\tilde{\delta}) \stackrel{\text { INT12 }}{=} \int_{v \in[0,1)}\left(\sigma_{\mathrm{abs}}^{v}(\tilde{a}) \cdot t^{\prime \prime}\right)+\int_{v \in[0,1)} \sigma_{\mathrm{abs}}^{v}(\tilde{\delta}) \stackrel{\text { SIA } 6}{=} \\
& \int_{v \in[0,1)}\left(\sigma_{\mathrm{abs}}^{v}(\tilde{a}) \cdot \bar{v}_{\mathrm{abs}}^{v}\left(t^{\prime \prime}\right)\right)+\int_{v \in[0,1)} \sigma_{\mathrm{abs}}^{v}(\tilde{\delta}) \stackrel{\text { INT6, Lemma 8 }}{=} \\
& \int_{v \in[0,1)}\left(\sigma_{\mathrm{abs}}^{v}(\tilde{a}) \cdot \sigma_{\mathrm{abs}}^{v}\left(t_{[v]}^{\prime \prime}\right)\right)+\int_{v \in[0,1)} \sigma_{\mathrm{abs}}^{v}(\tilde{\delta}) \stackrel{\mathrm{SAT} 5}{=} \\
& \int_{v \in[0,1)} \sigma_{\mathrm{abs}}^{v}\left(\tilde{a} \cdot \bar{v}_{\mathrm{abs}}^{0}\left(t_{[v]}^{\prime \prime}\right)\right)+\int_{v \in[0,1)} \sigma_{\mathrm{abs}}^{v}(\tilde{\delta}) \stackrel{\mathrm{INT} 11}{=} \\
& \int_{v \in[0,1)}\left(\sigma_{\mathrm{abs}}^{v}\left(\tilde{a} \cdot \bar{v}_{\mathrm{abs}}^{0}\left(t_{[v]}^{\prime \prime}\right)\right)+\sigma_{\mathrm{abs}}^{v}(\tilde{\delta})\right) \stackrel{\mathrm{SAT} 3}{=} \int_{v \in[0,1)} \sigma_{\mathrm{abs}}^{v}\left(\tilde{a} \cdot \bar{v}_{\mathrm{abs}}^{0}\left(t_{[v]}^{\prime \prime}\right)+\tilde{\delta}\right) \stackrel{\mathrm{SAU} 1}{=} \\
& \int_{v \in[0,1)} \sigma_{\mathrm{abs}}^{v}\left(\nu_{\mathrm{abs}}(\tilde{a}) \cdot \bar{v}_{\mathrm{abs}}^{0}\left(t_{[v]}^{\prime \prime}\right)+\tilde{\delta}\right) \stackrel{\mathrm{SAU} 4}{=} \int_{v \in[0,1)} \sigma_{\mathrm{abs}}^{v}\left(\nu_{\mathrm{abs}}\left(\tilde{a} \cdot \bar{v}_{\mathrm{abs}}^{0}\left(t_{[v]}^{\prime \prime}\right)\right)+\tilde{\delta}\right)
\end{aligned}
$$

Lemmas 7-10 are used to shorten the calculations in the proof of Theorem 12 . The following lemma is also used in the proof of that theorem.

Lemma 11 For $p \in[0,1)$, the equation $\bar{v}_{\text {abs }}^{p}(\underline{\delta})=\sigma_{\text {abs }}^{1}(\dot{\delta})$ is derivable from the axioms of $\mathrm{ACP}^{\mathrm{sat}} \mathrm{I} \checkmark$ and the explicit definition of the constants and operators in Table 25.

\section{Proof.}




$$
\begin{aligned}
& \bar{v}_{\mathrm{abs}}^{p}(\underline{\delta})=\bar{v}_{\mathrm{abs}}^{p}\left(\int_{v \in[0,1)} \sigma_{\mathrm{abs}}^{v}(\tilde{\delta})\right) \stackrel{\mathrm{SAI} 6}{=} \int_{v \in[0,1)} \bar{v}_{\mathrm{abs}}^{p}\left(\sigma_{\mathrm{abs}}^{v}(\tilde{\delta})\right) \stackrel{\mathrm{INT} 4}{=} \\
& \int_{v \in[0, p)} \bar{v}_{\mathrm{abs}}^{p}\left(\sigma_{\mathrm{abs}}^{v}(\tilde{\delta})\right)+\int_{v \in[p, 1)} \bar{v}_{\mathrm{abs}}^{p}\left(\sigma_{\mathrm{abs}}^{v}(\tilde{\delta})\right) \stackrel{\mathrm{SAI}_{3}, \mathrm{SAI} 3^{\prime}}{=} \\
& \int_{v \in[0, p)} \sigma_{\mathrm{abs}}^{v}\left(\bar{v}_{\mathrm{abs}}^{p-v}\left(\bar{v}_{\mathrm{abs}}^{0}(\tilde{\delta})\right)\right)+\int_{v \in[p, 1)} \sigma_{\mathrm{abs}}^{v}(\tilde{\delta}) \stackrel{\mathrm{SAI} 1, \mathrm{SAI} 2}{=} \\
& \int_{v \in[0, p)} \sigma_{\mathrm{abs}}^{v}\left(\sigma_{\mathrm{abs}}^{p-v}(\dot{\delta})\right)+\int_{v \in[p, 1)} \sigma_{\mathrm{abs}}^{v}(\tilde{\delta}) \stackrel{\mathrm{SAT} 2}{=} \int_{v \in[0, p)} \sigma_{\mathrm{abs}}^{p}(\dot{\delta})+\int_{v \in[p, 1)} \sigma_{\mathrm{abs}}^{v}(\tilde{\delta}) \stackrel{\text { INT } 5,8}{=} \\
& \sigma_{\text {abs }}^{p}(\dot{\delta})+\sigma_{\text {abs }}^{1}(\dot{\delta}) \stackrel{\text { SAT2,3, }}{=}{ }^{\mathrm{A} 6 \mathrm{ID}} \sigma_{\mathrm{abs}}^{1}(\dot{\delta})
\end{aligned}
$$

The existence of an embedding of $\mathrm{ACP}^{\mathrm{dat}} \sqrt{ }$ in $\mathrm{ACP}^{\mathrm{sat}} \mathrm{I} \checkmark$ is now established by proving the following theorem.

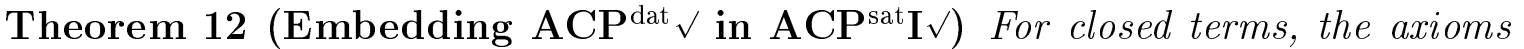
of $\mathrm{ACP}^{\mathrm{dat}} \sqrt{ }$ are derivable from the axioms of $\mathrm{ACP}^{\mathrm{sat}} \mathrm{I} \checkmark$ and the explicit definitions of the constants and operators $\underline{a}, \sigma_{\mathrm{abs}}, v_{\mathrm{abs}}, \bar{v}_{\mathrm{abs}}$ and $\sqrt{\mathrm{d}}_{\mathrm{in}}$ Table 25.

Proof. The proof of this theorem is given in Appendix A.2. The proof is a matter of straightforward calculations. Lemmas 1 and 3 (pages 8-8) and Lemmas 7-11 (page 4041) are very useful in the proof.

\section{Concluding remarks}

We presented real time and discrete time versions of ACP with both absolute timing and relative timing, starting with a new real time version of ACP with absolute timing called $\mathrm{ACP}^{\mathrm{sat}}$. We demonstrated that $\mathrm{ACP}^{\text {sat }}$ extended with integration and initial abstraction generalizes the presented real time version with relative timing and the presented discrete time version with absolute timing. We focussed on versions of ACP with timing where execution of actions and passage of time are separated, but explained how they can be combined in these versions. The material resulted from a systematic study of some of the most important issues relevant to dealing with time-dependent behaviour of processes - viz. absolute vs relative timing, continuous vs discrete time scale, and separation vs combination of execution of actions and passage of time - in the setting of ACP.

All real time and discrete time versions of ACP presented in this chapter include the immediate deadlock constant $\dot{\delta}$. This constant enables us to distinguish timing inconsistencies from incapabilities of performing actions as well as idling. This is certainly relevant to versions with absolute timing because timing inconsistencies readily arise. The usefulness of the immediate deadlock constant in practice is not yet clear for versions with relative timing. Minor adaptations of the versions of ACP with relative timing presented in this chapter are needed to obtain versions without the immediate deadlock constant.

The discrete time versions of ACP presented in this chapter are conservative extensions of the discrete time versions of [7]. The real time versions presented in this chapter, unlike the real time versions of [2] and [4], do not exclude the possibility of two or more actions to be performed consecutively at the same point in time. This feature seems to be essential to obtain simple and natural embeddings of discrete time versions as well as useful in practice when describing and analyzing distributed systems where entirely independent actions happen at different locations. 
We did not extend the different versions of ACP with timing presented in this chapter with recursion, abstraction, and other features that are important to make these versions suitable for being applied. This has been done for the earlier versions of ACP with timing referred to in this chapter. Some of those versions have been successfully used for describing and analyzing systems and protocols of various kinds, see e.g. [16], [27], [31], [32], [40], [41] and [45], as well as for defining semantics of programming and specification languages, see e.g. [12], [14] and [17].

We did not give explicit consideration to other algebraic concurrency theories that deal with time-dependent behaviour. In general, they have urgent actions and relative timing. This is, for example, the case with ATP [37], the different versions of CCS with timing [19, 35, 44] and TIC [39] - TIC is rooted in LOTOS [46]. We claim, on the basis of the connections described in [6], that there are indeed close connections between these theories and the versions of ACP with relative timing presented in this chapter, i.e. $\mathrm{ACP}^{\mathrm{srt}}$ and $\mathrm{ACP}{ }^{\mathrm{drt}}$. We also claim that there is a close connection between TPL [26] and ACP ${ }^{\text {drt }}$, only TPL is based on testing equivalence instead of bisimulation equivalence. Timed CSP [21], which is based on timed traces and timed failures, has urgent actions and relative timing as well. In [20], the CCS-like process algebras with timing of [26], [35], [36] and [44] are compared. For comprehensive overviews of existing algebraic concurrency theories that deal with time-dependent behaviour, having their roots in ACP, CCS, CSP, LOTOS or others, the reader is referred to Chapter 12 of [30] and Chapter 8 of [42]. These overviews include discussions of interesting connections with the versions of ACP with timing presented in earlier chapters of [30] and [42], respectively.

\section{References}

[1] L. Aceto, W.J. Fokkink, and C. Verhoef. Structural operational semantics. To appear in J.A. Bergstra, A. Ponse, and S.A. Smolka, editors, Handbook of Process Algebra. Elsevier, 2000.

[2] J.C.M. Baeten and J.A. Bergstra. Real time process algebra. Formal Aspects of Computing, 3(2):142-188, 1991.

[3] J.C.M. Baeten and J.A. Bergstra. Discrete time process algebra (extended abstract). In W.R. Cleaveland, editor, CONCUR'92, pages 401-420. LNCS 630, Springer-Verlag, 1992. Full version: Report P9208b, Programming Research Group, University of Amsterdam.

[4] J.C.M. Baeten and J.A. Bergstra. Real space process algebra. Formal Aspects of Computing, 5(6):481-529, 1993.

[5] J.C.M. Baeten and J.A. Bergstra. Discrete time process algebra with abstraction. In H. Reichel, editor, Fundamentals of Computation Theory, pages 1-15. LNCS 965, Springer-Verlag, 1995.

[6] J.C.M. Baeten and J.A. Bergstra. Real time process algebra with infinitesimals. In A. Ponse, C. Verhoef, and S.F.M. van Vlijmen, editors, Algebra of Communicating Processes 1994, pages 148-187. Workshop in Computing Series, Springer-Verlag, 1995. 
[7] J.C.M. Baeten and J.A. Bergstra. Discrete time process algebra. Formal Aspects of Computing, 8(2):188-208, 1996.

[8] J.C.M. Baeten and J.A. Bergstra. Discrete time process algebra: Absolute time, relative time and parametric time. Fundamenta Informaticae, 29(1/2):51-76, 1997.

[9] J.C.M. Baeten and C. Verhoef. A congruence theorem for structured operational semantics with predicates. In E. Best, editor, CONCUR'93, pages 477-492. LNCS 715, Springer-Verlag, 1993.

[10] J.C.M. Baeten and C. Verhoef. Concrete process algebra. In S. Abramsky, D. Gabbay, and T.S.E. Maibaum, editors, Handbook of Logic in Computer Science, Volume IV, pages 149-268. Oxford University Press, 1995.

[11] J.C.M. Baeten and W.P. Weijland. Process Algebra. Cambridge Tracts in Theoretical Computer Science 18, Cambridge University Press, 1990.

[12] J.A. Bergstra and P. Klint. The discrete time ToolBus - A software coordination architecture. Science of Computer Programming, 31:205-229, 1998.

[13] J.A. Bergstra and J.W. Klop. The algebra of recursively defined processes and the algebra of regular processes. In Proceedings 11th ICALP, pages 82-95. LNCS 172, Springer Verlag, 1984.

[14] J.A. Bergstra, C.A. Middelburg, and Y.S. Usenko. Discrete time process algebra and the semantics of SDL. CWI Report SEN-R9809, CWI, 1998. To appear in J.A. Bergstra, A. Ponse, and S.A. Smolka, editors, Handbook of Process Algebra. Elsevier, 2000.

[15] R.N. Bol and J.F. Groote. The meaning of negative premises in transition system specifications. Journal of the ACM, 43:863-914, 1996.

[16] S.H.J. Bos and M.A. Reniers. The $\mathrm{I}^{2} \mathrm{C}$-bus in discrete-time process algebra. Science of Computer Programming, 29:235-258, 1997.

[17] J. van den Brink and W.O.D. Griffioen. Formal semantics of discrete absolute timed interworkings. In A. Ponse, C. Verhoef, and S.F.M. van Vlijmen, editors, Algebra of Communicating Processes 1994, pages 106-123. Workshop in Computing Series, Springer-Verlag, 1995.

[18] S.D. Brookes, C.A.R. Hoare, and A.W. Roscoe. A theory of communicating sequential processes. Journal of the ACM, 31:560-599, 1984.

[19] L. Chen. An interleaving model for real-time systems. In A. Nerode and M. Taitslin, editors, Symposium on Logical Foundations of Computer Science, pages 81-92. LNCS 620, Springer-Verlag, 1992.

[20] F. Corradini, D. D'Ortenzio, and P. Inverardi. On the relationships among four timed process algebras. Fundamenta Informaticae, 38(4):377-395, 1999. 
[21] J. Davies et al. Timed CSP: Theory and practice. In J.W. de Bakker, C. Huizing, W.P. de Roever, and G. Rozenberg, editors, Real Time: Theory and Practice, pages 640-675. LNCS 600, Springer-Verlag, 1992.

[22] W..J. Fokkink. An elimination theorem for regular behaviours with integration. In E. Best, editor, CONCUR'93, pages 432-446. LNCS 715, Springer-Verlag, 1993.

[23] W.J. Fokkink and A.S. Klusener. An effective axiomatization for real time ACP. Information and Computation, 122:286-299, 1995.

[24] W.J. Fokkink and C. Verhoef. A conservative look at operational semantics with variable binding. Information and Computation, 146:24-54, 1998.

[25] J.F. Groote. Transition system specifications with negative premises. Theoretical Computer Science, 118:263-299, 1993.

[26] M. Hennessy and T. Regan. A process algebra for timed systems. Information and Computation, 117:221-239, 1995.

[27] J.A. Hillebrand. The ABP and CABP - a comparison of performances in real time process algebra. In A. Ponse, C. Verhoef, and S.F.M. van Vlijmen, editors, Algebra of Communicating Processes 1994, pages 124-147. Workshop in Computing Series, Springer-Verlag, 1995.

[28] C.A.R. Hoare. Communicating Sequential Processes. Prentice-Hall, 1985.

[29] A.S. Klusener. Completeness in real-time process algebra. In J.C.M. Baeten and J.F. Groote, editors, CONCUR'91, pages 376-392. LNCS 527, Springer-Verlag, 1991.

[30] A.S. Klusener. Models and Axioms for a Fragment of Real Time Process Algebra. PhD thesis, Eindhoven University of Technology, Department of Computing Science, 1993.

[31] M.J. Koens and L.H. Oei. A real time $\mu$ CRL specification of a system for traffic regulation at signalized intersections. In A. Ponse, C. Verhoef, and S.F.M. van Vlijmen, editors, Algebra of Communicating Processes 1994, pages 252-279. Workshop in Computing Series, Springer-Verlag, 1995.

[32] J.M.S. van den Meerendonk. Specification and verification of a circuit in $\mathrm{ACP}_{\mathrm{drt}}$-ID. M.Sc. Thesis, Eindhoven University of Technology, Department of Mathematics and Computing Science, 1996.

[33] R. Milner. A Calculus of Communicating Systems. LNCS 92, Springer-Verlag, 1980.

[34] R. Milner. Communication and Concurrency. Prentice-Hall, 1989.

[35] F. Moller and C. Tofts. A temporal calculus of communicating systems. In J.C.M. Baeten and J.W. Klop, editors, CONCUR'90, pages 401-415. LNCS 458, Springer-Verlag, 1990. 
[36] F. Moller and C. Tofts. Relating processes with respect to speed. In J.C.M. Baeten and J.F. Groote, editors, CONCUR'91, pages 424-438. LNCS 527, Springer-Verlag, 1991.

[37] X. Nicollin and J. Sifakis. The algebra of timed processes ATP: Theory and application. Information and Computation, 114:131-178, 1994.

[38] G.D. Plotkin. A structural approach to operational semantics. Technical Report DAIMI FN-19, University of Aarhus, Department of Computer Science, 1981.

[39] J. Quemada, D. de Frutos, and A. Azcorra. TIC: A timed calculus. Formal Aspects of Computing, 5(3):224-252, 1993.

[40] A. Stins and A. Schoneveld. Specification of a bank account with process algebra. Report P9307, University of Amsterdam, Programming Research Group, 1993.

[41] J.J. Vereijken. Fischer's protocol in timed process algebra. In A. Ponse, C. Verhoef, and S.F.M. van Vlijmen, editors, Algebra of Communicating Processes 1995, pages 245-284. Report 95-14, Eindhoven University of Technology, Department of Computing Science, 1995.

[42] J.J. Vereijken. Discrete Time Process Algebra. PhD thesis, Eindhoven University of Technology, Department of Computing Science, 1997.

[43] C. Verhoef. A congruence theorem for structured operational semantics with predicates and negative premises. Nordic Journal of Computing, 2:274-302, 1995.

[44] Wang Yi. Real-time behaviour of asynchronous agents. In J.C.M. Baeten and J.W. Klop, editors, CONCUR'90, pages 502-520. LNCS 458, Springer-Verlag, 1990 .

[45] A. van Waveren. Specification of remote sensing mechanisms in real space process algebra. Report P9220, University of Amsterdam, Programming Research Group, 1992.

[46] LOTOS - a formal description technique based on the temporal ordering of observational behaviour. International Standard ISO 8807, 1989.

\section{A Proofs of theorems}

\section{A.1 Theorem 6}

Theorem 6 (Embedding $\mathbf{A C P}^{\text {srt }}$ in $\mathrm{ACP}^{\mathrm{sat}} \sqrt{ }$ ) For closed terms, the axioms of $\mathrm{ACP}^{\mathrm{srt}}$ are derivable from the axioms of $\mathrm{ACP}^{\mathrm{sat}} \sqrt{ }$ and the explicit definitions of the constants and operators $\widetilde{\tilde{a}}, \sigma_{\text {rel }}, v_{\text {rel }}, \bar{v}_{\text {rel }}$, and $\nu_{\text {rel }}$ in Table 19.

\section{Proof.}


To begin with, we show that the axioms of $\mathrm{BPA}^{\text {srt }}$ are derivable for closed terms. Throughout this proof we do not expound the trivial cases.

SRT1 : $\quad \sigma_{\text {rel }}^{0}(t)=\sqrt{\mathrm{s}}_{\mathrm{s}} v \cdot \bar{v}_{\mathrm{abs}}^{v}(t) \stackrel{\text { IIAI }}{=} \sqrt{\mathrm{s}}_{\mathrm{s}} v \cdot t \stackrel{\text { SIA } 4}{=} t$

SRT2 : $\quad \sigma_{\text {rel }}^{p}\left(\sigma_{\text {rel }}^{q}(t)\right)=\sqrt{ }_{\mathrm{s}} v \cdot \bar{v}_{\text {abs }}^{v+p}\left(\sqrt{s}_{\mathrm{s}} w \cdot \bar{v}_{\text {abs }}^{w+q}(t)\right) \stackrel{\text { SIA } 2}{=} \sqrt{\mathrm{s}}_{\mathrm{s}} v \cdot \bar{v}_{\text {abs }}^{v+p}\left(\bar{v}_{\text {abs }}^{v+p+q}(t)\right) \stackrel{\text { SI } 2}{=}$ $\sqrt{\mathrm{s}}_{\mathrm{s}} v \cdot \bar{v}_{\mathrm{abs}}^{v+p+q}(t)=\sigma_{\mathrm{rel}}^{p+q}(t)$

SRT3 : $\quad \sigma_{\text {rel }}^{p}(t)+\sigma_{\text {rel }}^{p}\left(t^{\prime}\right)=\left(\sqrt{\mathrm{s}}_{\mathrm{s}} v \cdot \bar{v}_{\mathrm{abs}}^{v+p}(t)\right)+\left(\sqrt{\mathrm{s}}_{\mathrm{s}} w \cdot \bar{v}_{\mathrm{abs}}^{w+p}\left(t^{\prime}\right)\right) \stackrel{\text { IIA } 8}{=}$ $\sqrt{\mathrm{s}}_{\mathrm{s}} v \cdot\left(\bar{v}_{\mathrm{abs}}^{v+p}(t)+\bar{v}_{\mathrm{abs}}^{v}\left(\sqrt{\mathrm{s}}_{\mathrm{s}} w \cdot \bar{v}_{\mathrm{abs}}^{w+p}\left(t^{\prime}\right)\right) \stackrel{\text { SIA2 } 2}{=} \sqrt{\mathrm{s}}_{\mathrm{s}} v \cdot\left(\bar{v}_{\mathrm{abs}}^{v+p}(t)+\bar{v}_{\mathrm{abs}}^{v}\left(\bar{v}_{\mathrm{abs}}^{v+p}\left(t^{\prime}\right)\right)\right) \stackrel{\mathrm{SI} 2}{=}\right.$ $\sqrt{\mathrm{s}}_{\mathrm{s}} v \cdot\left(\bar{v}_{\mathrm{abs}}^{v+p}(t)+\bar{v}_{\mathrm{abs}}^{v+p}\left(t^{\prime}\right)\right) \stackrel{\mathrm{SAI} 4}{=} \sqrt{\mathrm{s}}_{\mathrm{s}} v \cdot \bar{v}_{\mathrm{abs}}^{v+p}\left(t+t^{\prime}\right)=\sigma_{\mathrm{rel}}^{p}\left(t+t^{\prime}\right)$

SRT4 : $\quad \sigma_{\text {rel }}^{p}(t) \cdot t^{\prime}=\left(\sqrt{\mathrm{s}}_{\mathrm{s}} v \cdot \bar{v}_{\mathrm{abs}}^{v+p}(t)\right) \cdot t^{\prime} \stackrel{\mathrm{SIA} 9}{=} \sqrt{\mathrm{s}}_{\mathrm{s}} v \cdot\left(\bar{v}_{\mathrm{abs}}^{v+p}(t) \cdot t^{\prime}\right) \stackrel{\mathrm{SAI} 5}{=} \sqrt{\mathrm{s}}_{\mathrm{s}} v \cdot \bar{v}_{\mathrm{abs}}^{v+p}\left(t \cdot t^{\prime}\right)=$ $\sigma_{\text {rel }}^{p}\left(t \cdot t^{\prime}\right)$

A6SRa $: \quad \tilde{\widetilde{a}}+\widetilde{\widetilde{\delta}}=\left(\sqrt{\mathrm{s}}_{v} v \cdot \sigma_{\mathrm{abs}}^{v}(\tilde{a})\right)+\left(\sqrt{\mathrm{s}}_{\mathrm{s}} w \cdot \sigma_{\mathrm{abs}}^{w}(\tilde{\delta})\right) \stackrel{\text { SIA } 8}{=}$

$\sqrt{\mathrm{s}}_{\mathrm{s}} v \cdot\left(\sigma_{\mathrm{abs}}^{v}(\tilde{a})+\bar{v}_{\mathrm{abs}}^{v}\left(\sqrt{\mathrm{s}}_{\mathrm{s}} w \cdot \sigma_{\mathrm{abs}}^{w}(\tilde{\delta})\right)\right) \stackrel{\text { SIA2 }}{=} \sqrt{\mathrm{s}}_{\mathrm{s}} v \cdot\left(\sigma_{\mathrm{abs}}^{v}(\tilde{a})+\bar{v}_{\mathrm{abs}}^{v}\left(\sigma_{\mathrm{abs}}^{v}(\tilde{\delta})\right)\right) \stackrel{\mathrm{SAII}^{\prime}}{=}$

$\sqrt{\mathrm{s}} v \cdot\left(\sigma_{\mathrm{abs}}^{v}(\tilde{a})+\sigma_{\mathrm{abs}}^{v}(\tilde{\delta})\right) \stackrel{\mathrm{SAT3}}{=} \sqrt{\mathrm{s}} v \cdot \sigma_{\mathrm{abs}}^{v}(\tilde{a}+\tilde{\delta}) \stackrel{\mathrm{A} 6 \mathrm{SAa}}{=} \sqrt{\mathrm{s}}_{\mathrm{s}} v \cdot \sigma_{\mathrm{abs}}^{v}(\tilde{a})=\tilde{\widetilde{a}}$

$\mathrm{A} 6 \mathrm{SRb}: \quad \sigma_{\mathrm{rel}}^{r}(t)+\tilde{\widetilde{\delta}}=\left(\sqrt{\mathrm{s}} v \cdot \bar{v}_{\mathrm{abs}}^{v+r}(t)\right)+\left(\sqrt{\mathrm{s}}_{\mathrm{s}} w \cdot \sigma_{\mathrm{abs}}^{w}(\tilde{\delta})\right) \stackrel{\text { SIA } 8}{=}$

$\sqrt{\mathrm{s}}_{\mathrm{s}} v \cdot\left(\bar{v}_{\mathrm{abs}}^{v+r}(t)+\bar{v}_{\mathrm{abs}}^{v}\left(\sqrt{\mathrm{s}}_{\mathrm{s}} w \cdot \sigma_{\mathrm{abs}}^{w}(\tilde{\delta})\right)\right) \stackrel{\mathrm{SIA}^{2}}{=} \sqrt{\mathrm{s}}_{\mathrm{s}} v \cdot\left(\bar{v}_{\mathrm{abs}}^{v+r}(t)+\bar{v}_{\mathrm{abs}}^{v}\left(\sigma_{\mathrm{abs}}^{v}(\tilde{\delta})\right)\right) \stackrel{\text { SAI3' }}{=}$

$\sqrt{\mathrm{s}}_{\mathrm{s}} v \cdot\left(\bar{v}_{\mathrm{abs}}^{v+r}(t)+\sigma_{\mathrm{abs}}^{v}(\tilde{\delta})\right) \stackrel{\text { Lemma } 4}{=} \sqrt{\mathrm{s}}_{\mathrm{s}} v \cdot\left(\sigma_{\mathrm{abs}}^{v+r}(t)+\sigma_{\mathrm{abs}}^{v}(\tilde{\delta})\right) \stackrel{\text { SAT2 }}{=}$

$\sqrt{\mathrm{s}}_{\mathrm{s}} v \cdot\left(\sigma_{\mathrm{abs}}^{v}\left(\sigma_{\mathrm{abs}}^{r}(t)\right)+\sigma_{\mathrm{abs}}^{v}(\tilde{\delta})\right) \stackrel{\mathrm{SAT} 3}{=} \sqrt{\mathrm{s}}_{\mathrm{s}} v \cdot \sigma_{\mathrm{abs}}^{v}\left(\sigma_{\mathrm{abs}}^{r}(t)+\tilde{\delta}\right) \stackrel{\mathrm{A} 6 \mathrm{SAb}}{=}$

$\sqrt{\mathrm{s}}_{\mathrm{s}} v \cdot \sigma_{\mathrm{abs}}^{v}\left(\sigma_{\mathrm{abs}}^{r}(t)\right) \stackrel{\mathrm{SAT} 2}{=} \sqrt{\mathrm{s}}_{\mathrm{s}} v \cdot \sigma_{\mathrm{abs}}^{v+r}(t) \stackrel{\text { Lemma } 4}{=} \sqrt{\mathrm{s}}_{\mathrm{s}} v \cdot \bar{v}_{\mathrm{abs}}^{v+r}(t)=\sigma_{\mathrm{rel}}^{r}(t)$

$\mathrm{A} 7 \mathrm{SR}: \quad \tilde{\tilde{\delta}} \cdot t=\left(\sqrt{\mathrm{s}}_{\mathrm{s}} v \cdot \sigma_{\mathrm{abs}}^{v}(\tilde{\delta})\right) \cdot t \stackrel{\text { SIA9 }}{=} \sqrt{\mathrm{s}}_{\mathrm{s}} v \cdot\left(\sigma_{\mathrm{abs}}^{v}(\tilde{\delta}) \cdot t\right) \stackrel{\text { SIA6 } 6}{=} \sqrt{\mathrm{s}}_{\mathrm{s}} v \cdot\left(\sigma_{\mathrm{abs}}^{v}(\tilde{\delta}) \cdot \bar{v}_{\mathrm{abs}}^{v}(t)\right) \stackrel{\text { Lemma } 4}{=}$ $\sqrt{\mathrm{s}}_{\mathrm{s}} v \cdot\left(\sigma_{\mathrm{abs}}^{v}(\tilde{\delta}) \cdot \sigma_{\mathrm{abs}}^{v}(t)\right) \stackrel{\mathrm{SAT} 5}{=} \sqrt{\mathrm{s}} v \cdot \sigma_{\mathrm{abs}}^{v}\left(\tilde{\delta} \cdot \bar{v}_{\mathrm{abs}}^{0}(t)\right) \stackrel{\text { A7SA }}{=} \sqrt{\mathrm{s}} v \cdot \sigma_{\mathrm{abs}}^{v}(\tilde{\delta})=\tilde{\tilde{\delta}}$

SRTO0 : $\quad v_{\text {rel }}^{p}(\dot{\delta})=\sqrt{\mathrm{s}}_{\mathrm{s}} v \cdot v_{\mathrm{abs}}^{v+p}\left(\bar{v}_{\mathrm{abs}}^{v}(\dot{\delta})\right) \stackrel{\mathrm{SAI0}}{=} \sqrt{\mathrm{s}}_{\mathrm{s}} v \cdot v_{\mathrm{abs}}^{v+p}\left(\sigma_{\mathrm{abs}}^{v}(\dot{\delta})\right) \stackrel{\text { SATO3 }}{=}$

$\sqrt{\mathrm{s}}_{\mathrm{s}} v \cdot \sigma_{\mathrm{abs}}^{v}\left(v_{\mathrm{abs}}^{p}(\dot{\delta})\right) \stackrel{\text { SATO0 }}{=} \sqrt{\mathrm{s}}_{\mathrm{s}} v \cdot \sigma_{\mathrm{abs}}^{v}(\dot{\delta}) \stackrel{\text { SAI0 }}{=} \sqrt{\mathrm{s}}_{\mathrm{s}} v \cdot \bar{v}_{\mathrm{abs}}^{v}(\dot{\delta}) \stackrel{\text { SIAI }}{=} \sqrt{\mathrm{s}}_{\mathrm{s}} v \cdot \dot{\delta} \stackrel{\mathrm{SIA}^{\prime}}{=} \dot{\delta}$

SRTO1: $\quad v_{\text {rel }}^{0}(t)=\sqrt{\mathrm{s}}_{v} v \cdot v_{\mathrm{abs}}^{v}\left(\bar{v}_{\mathrm{abs}}^{v}(t)\right) \stackrel{\text { SI4 }}{=} \sqrt{\mathrm{s}} v \cdot \sigma_{\mathrm{abs}}^{v}(\dot{\delta}) \stackrel{\text { SAI0 }}{=} \sqrt{\mathrm{s}} v \cdot \bar{v}_{\mathrm{abs}}^{v}(\dot{\delta}) \stackrel{\text { SIAI }}{=} \sqrt{\mathrm{s}} v \cdot \dot{\delta} \stackrel{\mathrm{SIA}^{\mathrm{II}} 4}{=} \dot{\delta}$

SRTO2 : $\quad v_{\mathrm{rel}}^{r}(\tilde{\widetilde{a}})=\sqrt{\mathrm{s}}_{\mathrm{s}} v \cdot v_{\mathrm{abs}}^{v+r}\left(\bar{v}_{\mathrm{abs}}^{v}\left(\sqrt{\mathrm{s}}_{\mathrm{s}} w \cdot \sigma_{\mathrm{abs}}^{w}(\tilde{a})\right)\right) \stackrel{\mathrm{SIA}^{2}}{=} \sqrt{\mathrm{s}}_{v} v \cdot v_{\mathrm{abs}}^{v+r}\left(\bar{v}_{\mathrm{abs}}^{v}\left(\sigma_{\mathrm{abs}}^{v}(\tilde{a})\right)\right) \stackrel{\mathrm{SAI}^{\prime}}{=}$ $\sqrt{\mathrm{s}} v \cdot v_{\mathrm{abs}}^{v+r}\left(\sigma_{\mathrm{abs}}^{v}(\tilde{a})\right) \stackrel{\mathrm{SATO} 3}{=} \sqrt{\mathrm{s}}_{\mathrm{s}} v \cdot \sigma_{\mathrm{abs}}^{v}\left(v_{\mathrm{abs}}^{r}(\tilde{a})\right) \stackrel{\mathrm{SATO} 2}{=} \sqrt{\mathrm{s}} v \cdot \sigma_{\mathrm{abs}}^{v}(\tilde{a})=\tilde{\widetilde{a}}$

SRTO3: $\quad v_{\mathrm{rel}}^{p+q}\left(\sigma_{\mathrm{rel}}^{p}(t)\right)=\sqrt{\mathrm{s}}_{\mathrm{s}} v \cdot v_{\mathrm{abs}}^{v+p+q}\left(\bar{v}_{\mathrm{abs}}^{v}\left(\sqrt{\mathrm{s}}_{\mathrm{s}} w \cdot \bar{v}_{\mathrm{abs}}^{w+p}(t)\right)\right) \stackrel{\text { SIA2 }}{=}$ $\sqrt{\mathrm{s}}_{\mathrm{s}} v \cdot v_{\mathrm{abs}}^{v+p+q}\left(\bar{v}_{\mathrm{abs}}^{v}\left(\bar{v}_{\mathrm{abs}}^{v+p}(t)\right)\right) \stackrel{\mathrm{SI2}}{=} \sqrt{\mathrm{s}}_{\mathrm{s}} v \cdot v_{\mathrm{abs}}^{v+p+q}\left(\bar{v}_{\mathrm{abs}}^{v+p}(t)\right)=$ Case $q=0: \stackrel{\text { SI }}{=} V_{\mathrm{s}} v \cdot \sigma_{\text {abs }}^{v+p}(\dot{\delta}) \stackrel{\text { SAIO }}{=} V_{\mathrm{s}} v \cdot \bar{v}_{\text {abs }}^{v+p}(\dot{\delta})=\sigma_{\text {rel }}^{p}(\dot{\delta}) \stackrel{\text { SRTO1 }}{=} \sigma_{\text {rel }}^{p}\left(v_{\text {rel }}^{q}(t)\right)$

Case $q>0: \stackrel{\mathrm{SI} 2}{=} \sqrt{\mathrm{s}} v \cdot v_{\mathrm{abs}}^{v+p+q}\left(\bar{v}_{\mathrm{abs}}^{v+p}\left(\bar{v}_{\mathrm{abs}}^{v+p}(t)\right)\right) \stackrel{\mathrm{SI} 1}{=}$ $\sqrt{\mathrm{s}}_{\mathrm{s}} v \cdot \bar{v}_{\mathrm{abs}}^{v+p}\left(v_{\mathrm{abs}}^{v+p+q}\left(\bar{v}_{\mathrm{abs}}^{v+p}(t)\right)\right) \stackrel{\stackrel{\mathrm{SIA} 2}{=}}{{ }_{\mathrm{s}}} v \cdot \bar{v}_{\mathrm{abs}}^{v+p}\left(\sqrt{\mathrm{s}}_{\mathrm{s}} w \cdot v_{\mathrm{abs}}^{w+q}\left(\bar{v}_{\mathrm{abs}}^{w}(t)\right)\right)=$ $\sigma_{\text {rel }}^{p}\left(v_{\text {rel }}^{q}(t)\right)$

SRTO4: $v_{\text {rel }}^{p}\left(t+t^{\prime}\right)=\sqrt{\mathrm{s}}_{\mathrm{s}} v \cdot v_{\mathrm{abs}}^{v+p}\left(\bar{v}_{\mathrm{abs}}^{v}\left(t+t^{\prime}\right)\right) \stackrel{\mathrm{SAI} 4}{=} \sqrt{\mathrm{s}}_{\mathrm{s}} v \cdot v_{\mathrm{abs}}^{v+p}\left(\bar{v}_{\mathrm{abs}}^{v}(t)+\bar{v}_{\mathrm{abs}}^{v}\left(t^{\prime}\right)\right) \stackrel{\text { SATO4 }}{=}$ $\sqrt{\mathrm{s}} v \cdot\left(v_{\mathrm{abs}}^{v+p}\left(\bar{v}_{\mathrm{abs}}^{v}(t)\right)+v_{\mathrm{abs}}^{v+p}\left(\bar{v}_{\mathrm{abs}}^{v}\left(t^{\prime}\right)\right)\right)=$

Case $p=0: \stackrel{\text { SI } 4, \mathrm{~A} 3}{=} \sqrt{\mathrm{s}}_{\mathrm{s}} v \cdot \sigma_{\mathrm{abs}}^{v}(\dot{\delta}) \stackrel{\text { SAIO, SIAI, SIA4 }}{=} \dot{\delta} \stackrel{\text { A3 }}{=} \dot{\delta}+\dot{\delta} \stackrel{\mathrm{SRTO}^{\prime} 1}{=} v_{\mathrm{rel}}^{p}(t)+v_{\mathrm{rel}}^{p}\left(t^{\prime}\right)$

Case $p>0: \stackrel{\text { SI } 2}{=} \sqrt{s}_{\mathrm{s}} v \cdot\left(v_{\mathrm{abs}}^{v+p}\left(\bar{v}_{\mathrm{abs}}^{v}(t)\right)+v_{\mathrm{abs}}^{v+p}\left(\bar{v}_{\mathrm{abs}}^{v}\left(\bar{v}_{\mathrm{abs}}^{v}\left(t^{\prime}\right)\right)\right) \stackrel{\stackrel{\mathrm{SI} 1}{=}}{=}\right.$ $\sqrt{\mathrm{s}}_{\mathrm{s}} v \cdot\left(v_{\mathrm{abs}}^{v+p}\left(\bar{v}_{\mathrm{abs}}^{v}(t)\right)+\bar{v}_{\mathrm{abs}}^{v}\left(v_{\mathrm{abs}}^{v+p}\left(\bar{v}_{\mathrm{abs}}^{v}\left(t^{\prime}\right)\right)\right)\right) \stackrel{\text { SIA } 2}{=}$

$\sqrt{s}_{\mathrm{s}} v \cdot\left(v_{\mathrm{abs}}^{v+p}\left(\bar{v}_{\mathrm{abs}}^{v}(t)\right)+\bar{v}_{\mathrm{abs}}^{v}\left(\sqrt{\mathrm{s}}_{\mathrm{s}} w \cdot v_{\mathrm{abs}}^{w+p}\left(\bar{v}_{\mathrm{abs}}^{w}\left(t^{\prime}\right)\right)\right) \stackrel{\text { SIA } 8}{=}\right.$

$\left(\sqrt{\mathrm{s}}_{\mathrm{s}} v \cdot v_{\mathrm{abs}}^{v+p}\left(\bar{v}_{\mathrm{abs}}^{v}(t)\right)\right)+\left(\sqrt{\mathrm{s}}_{\mathrm{s}} w \cdot v_{\mathrm{abs}}^{w+p^{2}}\left(\bar{v}_{\mathrm{abs}}^{w}\left(t^{\prime}\right)\right)\right)=v_{\mathrm{rel}}^{p}(t)+v_{\mathrm{rel}}^{p}\left(t^{\prime}\right)$

SRTO5 : $\quad v_{\mathrm{rel}}^{p}\left(t \cdot t^{\prime}\right)=\sqrt{\mathrm{s}}_{\mathrm{s}} v \cdot v_{\mathrm{abs}}^{v+p}\left(\bar{v}_{\mathrm{abs}}^{v}\left(t \cdot t^{\prime}\right)\right) \stackrel{\mathrm{SAI} 5}{=} \sqrt{\mathrm{s}} v \cdot v_{\mathrm{abs}}^{v+p}\left(\bar{v}_{\mathrm{abs}}^{v}(t) \cdot t^{\prime}\right) \stackrel{\mathrm{SATO5}}{=}$

$\sqrt{\mathrm{s}}_{\mathrm{s}} v \cdot\left(v_{\mathrm{abs}}^{v+p}\left(\bar{v}_{\mathrm{abs}}^{v}(t)\right) \cdot t^{\prime}\right) \stackrel{\text { SIA9 }}{=}\left(\sqrt{\mathrm{s}}_{\mathrm{s}} v \cdot v_{\mathrm{abs}}^{v+p}\left(\bar{v}_{\mathrm{abs}}^{v}(t)\right)\right) \cdot t^{\prime}=v_{\mathrm{rel}}^{p}(t) \cdot t^{\prime}$ 
SRI0 : $\quad \bar{v}_{\text {rel }}^{p}(\dot{\delta})=\sqrt{\mathrm{s}}_{\mathrm{s}} v \cdot \bar{v}_{\mathrm{abs}}^{v+p}\left(\bar{v}_{\mathrm{abs}}^{v}(\dot{\delta})\right) \stackrel{\text { SAI0a }}{=} \sqrt{\mathrm{s}}_{\mathrm{s}} v \cdot \bar{v}_{\mathrm{abs}}^{v+p}\left(\bar{v}_{\mathrm{abs}}^{v}\left(\bar{v}_{\mathrm{abs}}^{0}(\dot{\delta})\right)\right) \stackrel{\mathrm{SI9}}{=}$ $\sqrt{\mathrm{s}}_{\mathrm{s}} v \cdot \bar{v}_{\mathrm{abs}}^{v+p}\left(\bar{v}_{\mathrm{abs}}^{0}(\dot{\delta})\right) \stackrel{\text { SAIOa }}{=} \sqrt{\mathrm{s}}_{\mathrm{s}} v \cdot \bar{v}_{\text {abs }}^{v+p}(\dot{\delta})=\sigma_{\text {rel }}^{p}(\dot{\delta})$

SRI1 : $\quad \bar{v}_{\mathrm{rel}}^{0}(t)=\sqrt{\mathrm{s}}_{\mathrm{s}} v \cdot \bar{v}_{\mathrm{abs}}^{v}\left(\bar{v}_{\mathrm{abs}}^{v}(t)\right) \stackrel{\mathrm{SI} 2}{=} \sqrt{\mathrm{s}}_{\mathrm{s}} v \cdot \bar{v}_{\mathrm{abs}}^{v}(t) \stackrel{\text { IIAI }}{=} \sqrt{\mathrm{s}} v \cdot t \stackrel{\mathrm{SIA} 4}{=} t$

SRI2 : $\quad \bar{v}_{\mathrm{rel}}^{r}(\tilde{\widetilde{a}})=\sqrt{\mathrm{s}}_{\mathrm{s}} v \cdot \bar{v}_{\mathrm{abs}}^{v+r}\left(\bar{v}_{\mathrm{abs}}^{v}\left(\sqrt{\mathrm{s}}_{\mathrm{s}} w \cdot \sigma_{\mathrm{abs}}^{w}(\tilde{a})\right)\right) \stackrel{\mathrm{SIA} 2}{=} \sqrt{\mathrm{s}}_{\mathrm{s}} v \cdot \bar{v}_{\mathrm{abs}}^{v+r}\left(\bar{v}_{\mathrm{abs}}^{v}\left(\sigma_{\mathrm{abs}}^{v}(\tilde{a})\right)\right) \stackrel{\mathrm{SAI}^{\prime}}{=}$ $\sqrt{\mathrm{s}}_{\mathrm{s}} v \cdot \bar{v}_{\mathrm{abs}}^{v+r}\left(\sigma_{\mathrm{abs}}^{v}(\widetilde{a})\right) \stackrel{\mathrm{SAI} 3}{=} \sqrt{\mathrm{s}}_{\mathrm{s}} v \cdot \sigma_{\mathrm{abs}}^{v}\left(\bar{v}_{\mathrm{abs}}^{r}\left(\bar{v}_{\mathrm{abs}}^{0}(\tilde{a})\right)\right) \stackrel{\mathrm{SAI} 1}{=} \sqrt{\mathrm{s}} v \cdot \sigma_{\mathrm{abs}}^{v}\left(\bar{v}_{\mathrm{abs}}^{r}(\tilde{a})\right) \stackrel{\mathrm{SAI} 2}{=}$ $\sqrt{\mathrm{s}}_{\mathrm{s}} v \cdot \sigma_{\mathrm{abs}}^{v}\left(\sigma_{\mathrm{abs}}^{r}(\dot{\delta})\right) \stackrel{\mathrm{SAT}^{2}}{=} \sqrt{\mathrm{s}}_{\mathrm{s}} v \cdot \sigma_{\mathrm{abs}}^{v+r}(\dot{\delta}) \stackrel{\text { SAIO }}{=} \sqrt{\mathrm{s}}_{\mathrm{s}} v \cdot \bar{v}_{\mathrm{abs}}^{v+r}(\dot{\delta})=\sigma_{\mathrm{rel}}^{r}(\dot{\delta})$

SRI3 : $\quad \bar{v}_{\text {rel }}^{p+q}\left(\sigma_{\text {rel }}^{p}(t)\right)=\sqrt{\mathrm{s}}_{\mathrm{s}} v \cdot \bar{v}_{\mathrm{abs}}^{v+p+q}\left(\bar{v}_{\mathrm{abs}}^{v}\left(\sqrt{\mathrm{s}}_{\mathrm{s}} w \cdot \bar{v}_{\mathrm{abs}}^{w+p}(t)\right)\right) \stackrel{\text { SIA2 }}{=}$

$\sqrt{\mathrm{s}}_{\mathrm{s}} v \cdot \bar{v}_{\mathrm{abs}}^{v+p+q}\left(\bar{v}_{\mathrm{abs}}^{v}\left(\bar{v}_{\mathrm{abs}}^{v+p}(t)\right)\right) \stackrel{\mathrm{SI} 2}{=} \sqrt{\mathrm{s}}_{\mathrm{v}} v \cdot \bar{v}_{\mathrm{abs}}^{v+p}\left(\bar{v}_{\mathrm{abs}}^{v+p+q}\left(\bar{v}_{\mathrm{abs}}^{v+p}(t)\right)\right) \stackrel{\mathrm{SIA} 2}{=}$ $\sqrt{\mathrm{s}}_{\mathrm{s}} v \cdot \bar{v}_{\mathrm{abs}}^{v+p}\left(\sqrt{\mathrm{s}}_{\mathrm{s}} w \cdot \bar{v}_{\mathrm{abs}}^{w+q}\left(\bar{v}_{\mathrm{abs}}^{w}(t)\right)\right)=\sigma_{\mathrm{rel}}^{p}\left(\bar{v}_{\mathrm{rel}}^{q}(t)\right)$

SRI4 : $\quad \bar{v}_{\text {rel }}^{p}\left(t+t^{\prime}\right)=\sqrt{\mathrm{s}}_{\mathrm{s}} v \cdot \bar{v}_{\mathrm{abs}}^{v+p}\left(\bar{v}_{\mathrm{abs}}^{v}\left(t+t^{\prime}\right)\right) \stackrel{\mathrm{SAI} 4}{=}$

$\sqrt{\mathrm{s}}_{\mathrm{s}} v \cdot\left(\bar{v}_{\mathrm{abs}}^{v+p}\left(\bar{v}_{\mathrm{abs}}^{v}(t)\right)+\bar{v}_{\mathrm{abs}}^{v+p}\left(\bar{v}_{\mathrm{abs}}^{v}\left(t^{\prime}\right)\right)\right) \stackrel{\mathrm{SI} 2}{=}$

$\sqrt{\mathrm{s}}_{\mathrm{s}} v \cdot\left(\bar{v}_{\mathrm{abs}}^{v+p}\left(\bar{v}_{\mathrm{abs}}^{v}(t)\right)+\bar{v}_{\mathrm{abs}}^{v}\left(\bar{v}_{\mathrm{abs}}^{v+p}\left(\bar{v}_{\mathrm{abs}}^{v}\left(t^{\prime}\right)\right)\right)\right) \stackrel{\mathrm{SIA} 2}{=}$

$\sqrt{\mathrm{s}}_{\mathrm{s}} v \cdot\left(\bar{v}_{\mathrm{abs}}^{v+p}\left(\bar{v}_{\mathrm{abs}}^{v}(t)\right)+\bar{v}_{\mathrm{abs}}^{v}\left(\sqrt{\mathrm{s}}_{\mathrm{s}} w \cdot \bar{v}_{\mathrm{abs}}^{w+p}\left(\bar{v}_{\mathrm{abs}}^{w}\left(t^{\prime}\right)\right)\right)\right) \stackrel{\text { SIA } 8}{=}$

$\left(\sqrt{\mathrm{s}}_{\mathrm{s}} v \cdot \bar{v}_{\mathrm{abs}}^{v+p}\left(\bar{v}_{\mathrm{abs}}^{v}(t)\right)\right)+\left(\sqrt{\mathrm{s}}_{\mathrm{s}} w \cdot \bar{v}_{\mathrm{abs}}^{w+p}\left(\bar{v}_{\mathrm{abs}}^{w}\left(t^{\prime}\right)\right)\right)=\bar{v}_{\mathrm{rel}}^{p}(t)+\bar{v}_{\mathrm{rel}}^{p}\left(t^{\prime}\right)$

SRI5 : $\quad \bar{v}_{\mathrm{rel}}^{p}\left(t \cdot t^{\prime}\right)=\sqrt{\mathrm{s}}_{\mathrm{s}} v \cdot \bar{v}_{\mathrm{abs}}^{v+p}\left(\bar{v}_{\mathrm{abs}}^{v}\left(t \cdot t^{\prime}\right)\right) \stackrel{\text { SAI5 }}{=} \sqrt{\mathrm{s}}_{\mathrm{s}} v \cdot\left(\bar{v}_{\mathrm{abs}}^{v+p}\left(\bar{v}_{\mathrm{abs}}^{v}(t)\right) \cdot t^{\prime}\right) \stackrel{\text { SIA9 }}{=}$ $\left(\sqrt{\mathrm{s}}_{\mathrm{s}} v \cdot \bar{v}_{\mathrm{abs}}^{v+p}\left(\bar{v}_{\mathrm{abs}}^{v}(t)\right)\right) \cdot t^{\prime}=\bar{v}_{\mathrm{rel}}^{p}(t) \cdot t^{\prime}$

Next, we show that the additional axioms for $\mathrm{ACP}^{\text {srt }}$ are derivable for closed terms.

CF1SR: $\quad \tilde{\widetilde{a}}\left|\widetilde{\tilde{b}}=\left(\sqrt{\mathrm{s}}_{\mathrm{s}} v \cdot \sigma_{\mathrm{abs}}^{v}(\tilde{a})\right)\right|\left(\sqrt{\mathrm{s}}_{\mathrm{s}} v \cdot \sigma_{\mathrm{abs}}^{v}(\tilde{b})\right) \stackrel{\text { DISTR } 1, \text { SACM5 }}{=} \sqrt{\mathrm{s}}_{\mathrm{s}} v \cdot \sigma_{\mathrm{abs}}^{v}(\tilde{a} \mid \tilde{b}) \stackrel{\text { CF1SA }}{=}$ $\sqrt{\mathrm{s}}_{\mathrm{s}} v \cdot \sigma_{\mathrm{abs}}^{v}(\tilde{c})=\widetilde{\widetilde{c}}$ if $\gamma(a, b)=c$

CF2SR : The proof is similar to the proof of axiom CF1SR - axiom CF2SA is used instead of axiom CF1SA.

CM2SRID : $\quad \tilde{a} \Perp(t+\tilde{\widetilde{\delta}})=\left(\sqrt{s}_{\mathrm{s}} v \cdot \sigma_{\mathrm{abs}}^{v}(\tilde{a})\right) \Perp\left(t+\left(\sqrt{\mathrm{s}}_{v} v \cdot \sigma_{\mathrm{abs}}^{v}(\tilde{\delta})\right)\right) \stackrel{\text { SIA8, DISTR } \Perp}{=}$ $\sqrt{\mathrm{s}}_{\mathrm{s}} v \cdot\left(\sigma_{\mathrm{abs}}^{v}(\tilde{a}) \nVdash\left(\bar{v}_{\mathrm{abs}}^{v}(t)+\sigma_{\mathrm{abs}}^{v}(\tilde{\delta})\right)\right) \stackrel{\text { Lemma } 4}{=}$

$\sqrt{\mathrm{s}}_{\mathrm{s}} v \cdot\left(\sigma_{\mathrm{abs}}^{v}(\tilde{a}) \amalg\left(\sigma_{\mathrm{abs}}^{v}(t)+\sigma_{\mathrm{abs}}^{v}(\tilde{\delta})\right)\right) \stackrel{\text { SAT3, SACM } 2}{=}$

$\sqrt{\mathrm{s}}_{\mathrm{s}} v \cdot \sigma_{\mathrm{abs}}^{v}(\tilde{a} \|(t+\tilde{\delta})) \stackrel{\mathrm{CM} 2 \mathrm{SA}}{=} \sqrt{\mathrm{s}}_{v} v \sigma_{\mathrm{abs}}^{v}(\tilde{a} \cdot(t+\tilde{\delta})) \stackrel{\mathrm{SAI} 1, \mathrm{SAT} 1, \mathrm{SIA} 6}{=}$

$\sqrt{\mathrm{s}}_{\mathrm{s}} v \cdot \sigma_{\mathrm{abs}}^{v}\left(\tilde{a} \cdot \bar{v}_{\mathrm{abs}}^{0}(t+\tilde{\delta})\right) \stackrel{\mathrm{SAT5}}{=} \sqrt{\mathrm{s}}_{\mathrm{s}} v \cdot\left(\sigma_{\mathrm{abs}}^{v}(\tilde{a}) \cdot \sigma_{\mathrm{abs}}^{v}(t+\tilde{\delta})\right) \stackrel{\text { SAT3, Lemma } 4}{=}$

$\sqrt{\mathrm{s}} v \cdot\left(\sigma_{\mathrm{abs}}^{v}(\tilde{a}) \cdot\left(\bar{v}_{\mathrm{abs}}^{v}(t)+\sigma_{\mathrm{abs}}^{v}(\tilde{\delta})\right)\right) \stackrel{\mathrm{SAI} 3^{\prime}}{=}$

$\sqrt{\mathrm{s}}_{\mathrm{s}} v \cdot\left(\sigma_{\mathrm{abs}}^{v}(\tilde{a}) \cdot\left(\bar{v}_{\mathrm{abs}}^{v}(t)+\bar{v}_{\mathrm{abs}}^{v}\left(\sigma_{\mathrm{abs}}^{v}(\tilde{\delta})\right)\right)\right) \stackrel{\text { SIA } 2}{=}$

$\sqrt{\mathrm{s}}_{\mathrm{s}} v \cdot\left(\sigma_{\mathrm{abs}}^{v}(\tilde{a}) \cdot\left(\bar{v}_{\mathrm{abs}}^{v}(t)+\bar{v}_{\mathrm{abs}}^{v}\left(\sqrt{\mathrm{s}}_{\mathrm{s}} w \cdot \sigma_{\mathrm{abs}}^{w}(\tilde{\delta})\right)\right)\right) \stackrel{\mathrm{SAI} 4}{=}$

$\sqrt{\mathrm{s}}_{\mathrm{s}} v \cdot\left(\sigma_{\mathrm{abs}}^{v}(\tilde{a}) \cdot \bar{v}_{\mathrm{abs}}^{v}\left(t+\sqrt{\mathrm{s}}_{\mathrm{s}} w \cdot \sigma_{\mathrm{abs}}^{w}(\tilde{\delta})\right)\right) \stackrel{\text { SIA } 6}{=}$

$\sqrt{\mathrm{s}}_{\mathrm{s}} v \cdot\left(\sigma_{\mathrm{abs}}^{v}(\tilde{a}) \cdot\left(t+\sqrt{\mathrm{s}}_{\mathrm{s}} w \cdot \sigma_{\mathrm{abs}}^{w}(\tilde{\delta})\right)\right) \stackrel{\text { IIA9 }}{=}$

$\left(\sqrt{\mathrm{s}}_{\mathrm{s}} v \cdot \sigma_{\mathrm{abs}}^{v}(\tilde{a})\right) \cdot\left(t+\sqrt{\mathrm{s}} w \cdot \sigma_{\mathrm{abs}}^{w}(\tilde{\delta})\right)=\tilde{\widetilde{a}} \cdot(t+\tilde{\tilde{\delta}})$

CM3SRID : The proof is similar to the proof of axiom CM2SRID - axiom CM3SA is used instead of axiom CM2SA. 
SRCM1ID : $\quad \sigma_{\text {rel }}^{r}(t) \Perp\left(\nu_{\text {rel }}\left(t^{\prime}\right)+\tilde{\widetilde{\delta}}\right)=$

$$
\begin{aligned}
& \left(\sqrt{\mathrm{s}}_{\mathrm{s}} v \cdot \bar{v}_{\mathrm{abs}}^{v+r}(t)\right) \amalg\left(\left(\sqrt{\mathrm{s}} v \cdot \sigma_{\mathrm{abs}}^{v}\left(\nu_{\mathrm{abs}}\left(t^{\prime}\right)\right)\right)+\left(\sqrt{\mathrm{s}}_{\mathrm{s}} v \cdot \sigma_{\mathrm{abs}}^{v}(\tilde{\delta})\right)\right) \stackrel{\text { DISTR }}{=} \\
& \sqrt{\mathrm{s}}_{\mathrm{s}} v \cdot\left(\bar{v}_{\mathrm{abs}}^{v+r}(t) \amalg\left(\sigma_{\mathrm{abs}}^{v}\left(\nu_{\mathrm{abs}}\left(t^{\prime}\right)\right)+\sigma_{\mathrm{abs}}^{v}(\tilde{\delta})\right)\right) \stackrel{\text { Lemma } 4, \text { SAT3 }}{=} \\
& \sqrt{\mathrm{s}}_{\mathrm{s}} v \cdot\left(\sigma_{\mathrm{abs}}^{v+r}(t) \amalg \sigma_{\mathrm{abs}}^{v}\left(\nu_{\mathrm{abs}}\left(t^{\prime}\right)+\tilde{\delta}\right)\right) \stackrel{\mathrm{SACM} 1^{\prime}}{=} \sqrt{\mathrm{s}}_{\mathrm{s}} v \cdot \sigma_{\mathrm{abs}}^{v}(\tilde{\delta})=\widetilde{\delta}
\end{aligned}
$$

SRCM2ID : $\sigma_{\text {rel }}^{p}(t) \Downarrow\left(v_{\text {rel }}^{p}\left(t^{\prime}\right)+\sigma_{\text {rel }}^{p}\left(t^{\prime \prime}\right)\right)=$

$$
\begin{aligned}
& \left(\sqrt{\mathrm{s}}_{\mathrm{s}} v \cdot \bar{v}_{\mathrm{abs}}^{v+p}(t)\right) \|\left(\left(\sqrt{\mathrm{s}}_{\mathrm{s}} v \cdot v_{\mathrm{abs}}^{v+p}\left(\bar{v}_{\mathrm{abs}}^{v}\left(t^{\prime}\right)\right)\right)+\left(\sqrt{\mathrm{s}}_{\mathrm{s}} v \cdot \bar{v}_{\mathrm{abs}}^{v+p}\left(t^{\prime \prime}\right)\right)\right) \stackrel{\text { DISTR }}{=} \\
& \sqrt{\mathrm{s}}_{\mathrm{s}} v \cdot\left(\bar{v}_{\mathrm{abs}}^{v+p}(t) \|\left(v_{\mathrm{abs}}^{v+p}\left(\bar{v}_{\mathrm{abs}}^{v}\left(t^{\prime}\right)\right)+\bar{v}_{\mathrm{abs}}^{v+p}\left(t^{\prime \prime}\right)\right)\right) \stackrel{\text { Lemma } 4}{=} \\
& \sqrt{\mathrm{s}} v \cdot\left(\sigma_{\mathrm{abs}}^{v+p}(t) \Perp\left(v_{\mathrm{abs}}^{v+p}\left(\bar{v}_{\mathrm{abs}}^{v}\left(t^{\prime}\right)\right)+\sigma_{\mathrm{abs}}^{v+p}\left(t^{\prime \prime}\right)\right)\right) \stackrel{\mathrm{SACM} 2}{=} \\
& \sqrt{\mathrm{s}}_{\mathrm{s}} v \cdot \sigma_{\mathrm{abs}}^{v+p}\left(t \Perp t^{\prime \prime}\right) \stackrel{\text { Lemma } 4}{=} \sqrt{\mathrm{s}} v \cdot \bar{v}_{\mathrm{abs}}^{v+p}\left(t \Perp t^{\prime \prime}\right)=\sigma_{\text {rel }}^{p}\left(t \Perp t^{\prime \prime}\right)
\end{aligned}
$$

CM5SR : $\quad \tilde{a} \cdot t\left|\tilde{b}=\left(\left(\sqrt{\mathrm{s}}_{\mathrm{s}} v \cdot \sigma_{\mathrm{abs}}^{v}(\tilde{a})\right) \cdot t\right)\right|\left(\sqrt{\mathrm{s}}_{\mathrm{s}} v \cdot \sigma_{\mathrm{abs}}^{v}(\tilde{b})\right) \stackrel{\text { SIA9, DISTR } \mid}{=}$

CM6SR and CM7SR :

The proofs are similar to the proof of axiom CM5SR - axioms CM6SA and CM7SA are used instead of axiom CM5SA.

SRCM3ID : $\quad\left(\nu_{\text {rel }}(t)+\tilde{\widetilde{\delta}}\right) \mid \sigma_{\text {rel }}^{r}\left(t^{\prime}\right)=$

$$
\begin{aligned}
& \left(\left(\sqrt{s}_{\mathrm{s}} v \cdot \sigma_{\mathrm{abs}}^{v}\left(\nu_{\mathrm{abs}}(t)\right)\right)+\left(\sqrt{\mathrm{s}}_{\mathrm{s}} v \cdot \sigma_{\mathrm{abs}}^{v}(\tilde{\delta})\right)\right) \mid\left(\sqrt{\mathrm{s}}_{\mathrm{s}} v \cdot \bar{v}_{\mathrm{abs}}^{v+r}(t)\right) \stackrel{\text { DISTR }}{=} \\
& \sqrt{\mathrm{s}}_{\mathrm{s}} v \cdot\left(\left(\sigma_{\mathrm{abs}}^{v}\left(\nu_{\mathrm{abs}}(t)\right)+\sigma_{\mathrm{abs}}^{v}(\tilde{\delta})\right) \mid \bar{v}_{\mathrm{abs}}^{v+r}(t)\right) \stackrel{\text { SAT3, Lemma } 4}{=} \\
& \sqrt{\mathrm{s}}_{\mathrm{s}} v \cdot\left(\sigma_{\mathrm{abs}}^{v}\left(\nu_{\mathrm{abs}}(t)+\tilde{\delta}\right) \mid \sigma_{\mathrm{abs}}^{v+r}(t)\right) \stackrel{\mathrm{SACM}^{\prime}}{=} \sqrt{\mathrm{s}}_{\mathrm{s}} v \cdot \sigma_{\mathrm{abs}}^{v}(\tilde{\delta})=\tilde{\tilde{\delta}}
\end{aligned}
$$

SRCM4ID : The proof is similar to the proof of axiom SRCM3ID - axiom SACM4' is used instead of axiom SACM $3^{\prime}$.

SRCM5 : $\quad \sigma_{\text {rel }}^{p}(t)\left|\sigma_{\text {rel }}^{p}\left(t^{\prime}\right)=\left(\sqrt{s}_{\mathrm{s}} v \cdot \bar{v}_{\text {abs }}^{v+p}(t)\right)\right|\left(\sqrt{\mathrm{s}}_{\mathrm{s}} v \cdot \bar{v}_{\text {abs }}^{v+p}\left(t^{\prime}\right)\right) \stackrel{\text { DISTR|, SI11 }}{=}$ $\sqrt{\mathrm{s}}_{\mathrm{s}} v \cdot \bar{v}_{\mathrm{abs}}^{v+p}\left(t \mid t^{\prime}\right)=\sigma_{\text {rel }}^{p}\left(t \mid t^{\prime}\right)$

D1SR : $\quad \partial_{H}(\tilde{\tilde{a}})=\partial_{H}\left(\sqrt{s}_{\mathrm{s}} v \cdot \sigma_{\mathrm{abs}}^{v}(\tilde{a})\right) \stackrel{\mathrm{SIA} 15, \mathrm{SAD}}{=} \sqrt{\mathrm{s}} v \cdot \sigma_{\mathrm{abs}}^{v}\left(\partial_{H}(\tilde{a})\right) \stackrel{\mathrm{D} 1 \mathrm{SA}}{=}$ $\sqrt{\mathrm{s}}_{\mathrm{s}} v \cdot \sigma_{\mathrm{abs}}^{v}(\tilde{a})=\widetilde{\widetilde{a}} \quad$ if $a \notin H$

D2SR : $\quad$ The proof is similar to the proof of axiom D1SR - axiom D2SA is used instead of axioms D1SA.

SRD : $\quad \partial_{H}\left(\sigma_{\text {rel }}^{p}(t)\right)=\partial_{H}\left(\sqrt{ }_{\mathbf{s}} v \cdot \bar{v}_{\text {abs }}^{v+p}(t)\right) \stackrel{\text { SIA15, Lemma } 4 \text {, SAD }}{=} \sqrt{s}_{\mathbf{s}} v \cdot \bar{v}_{\text {abs }}^{v+p}\left(\partial_{H}(t)\right)=$ $\sigma_{\text {rel }}^{p}\left(\partial_{H}(t)\right)$ 


\begin{tabular}{|c|c|}
\hline SRU0 : & $\nu_{\text {rel }}(\dot{\delta})=\sqrt{\mathrm{s}}_{\mathrm{s}} v \cdot \sigma_{\mathrm{abs}}^{v}\left(\nu_{\mathrm{abs}}(\dot{\delta})\right) \stackrel{\text { SAU0,SAI0 }}{=} \sqrt{\mathrm{s}}_{\mathrm{s}} v \cdot \bar{v}_{\mathrm{abs}}^{v}(\dot{\delta}) \stackrel{\text { SIAI, SIA4 } 4}{=} \dot{\delta}$ \\
\hline SRU1 : & $\begin{array}{l}\nu_{\mathrm{rel}}(\tilde{\widetilde{a}})=\sqrt{\mathrm{s}}_{\mathrm{s}} v \cdot \sigma_{\mathrm{abs}}^{v}\left(\nu_{\mathrm{abs}}\left(\sqrt{\mathrm{s}}_{\mathrm{s}} w \cdot \sigma_{\mathrm{abs}}^{w}(\tilde{a})\right)\right) \stackrel{\operatorname{SIA} 16}{=} \\
\sqrt{\mathrm{s}}_{\mathrm{s}} v \cdot \sigma_{\mathrm{abs}}^{v}\left(\sqrt{\mathrm{s}}_{\mathrm{s}} w \cdot \nu_{\mathrm{abs}}\left(\sigma_{\mathrm{abs}}^{w}(\tilde{a})\right)\right) \stackrel{\operatorname{SIA7}}{=} \sqrt{\mathrm{s}}_{\mathrm{s}} v \cdot \sigma_{\mathrm{abs}}^{v}\left(\nu_{\mathrm{abs}}\left(\sigma_{\mathrm{abs}}^{0}(\tilde{a})\right)\right) \stackrel{\text { SAT1,SAI1 }}{=} \\
\sqrt{\mathrm{s}}_{\mathrm{s}} v \cdot \sigma_{\mathrm{abs}}^{v}\left(\nu_{\mathrm{abs}}(\tilde{a})\right) \stackrel{\operatorname{SAU} 1}{=} \sqrt{\mathrm{s}}_{\mathrm{s}} v \cdot \sigma_{\mathrm{abs}}^{v}(\tilde{a})=\tilde{\widetilde{a}}\end{array}$ \\
\hline SRU2 : & 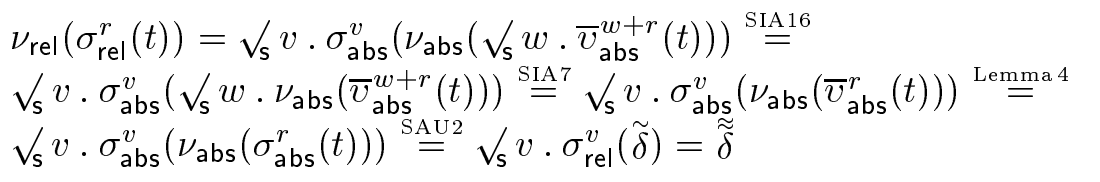 \\
\hline SRU3 : & $\begin{array}{l}\nu_{\mathrm{rel}}\left(t+t^{\prime}\right)=\sqrt{\mathrm{s}}_{\mathrm{s}} v \cdot \sigma_{\mathrm{abs}}^{v}\left(\nu_{\mathrm{abs}}\left(t+t^{\prime}\right)\right) \stackrel{\mathrm{SAU} 3, \mathrm{SAT} 3}{=} \\
\sqrt{\mathrm{s}}_{\mathrm{s}} v \cdot\left(\sigma_{\mathrm{abs}}^{v}\left(\nu_{\mathrm{abs}}(t)\right)+\sigma_{\mathrm{abs}}^{v}\left(\nu_{\mathrm{abs}}\left(t^{\prime}\right)\right)\right) \stackrel{\mathrm{SAI} 3^{\prime}}{=} \\
\sqrt{\mathrm{s}} v \cdot\left(\sigma_{\mathrm{abs}}^{v}\left(\nu_{\mathrm{abs}}(t)\right)+\bar{v}_{\mathrm{abs}}^{v}\left(\sigma_{\mathrm{abs}}^{v}\left(\nu_{\mathrm{abs}}\left(t^{\prime}\right)\right)\right)\right) \stackrel{\mathrm{SIA} 2}{=} \\
\sqrt{\mathrm{s}} v \cdot\left(\sigma_{\mathrm{abs}}^{v}\left(\nu_{\mathrm{abs}}(t)\right)+\bar{v}_{\mathrm{abs}}^{v}\left(\sqrt{\mathrm{s}}_{\mathrm{s}} w \cdot \sigma_{\mathrm{abs}}^{w}\left(\nu_{\mathrm{abs}}\left(t^{\prime}\right)\right)\right)\right) \stackrel{\mathrm{SIA} 8}{=} \\
\left(\sqrt{\mathrm{s}}_{\mathrm{s}} v \cdot \sigma_{\mathrm{abs}}^{v}\left(\nu_{\mathrm{abs}}(t)\right)\right)+\left(\sqrt{\mathrm{s}} w \cdot \sigma_{\mathrm{abs}}^{w}\left(\nu_{\mathrm{abs}}\left(t^{\prime}\right)\right)\right)=\nu_{\mathrm{rel}}(t)+\nu_{\mathrm{rel}}\left(t^{\prime}\right)\end{array}$ \\
\hline SRU4: & $\begin{array}{l}\nu_{\mathrm{rel}}\left(t \cdot t^{\prime}\right)=\sqrt{\mathrm{s}} v \cdot \sigma_{\mathrm{abs}}^{v}\left(\nu_{\mathrm{abs}}\left(t \cdot t^{\prime}\right)\right) \stackrel{\mathrm{SAU} 4}{=} \sqrt{\mathrm{s}}_{\mathrm{s}} v \cdot \sigma_{\mathrm{abs}}^{v}\left(\nu_{\mathrm{abs}}(t) \cdot t^{\prime}\right) \stackrel{\text { Lemma } 5}{=} \\
\sqrt{\mathrm{s}}_{\mathrm{s}} v \cdot\left(\sigma_{\mathrm{abs}}^{v}\left(\nu_{\mathrm{abs}}(t)\right) \cdot t^{\prime}\right) \stackrel{\text { SAA9 }}{=}\left(\sqrt{\mathrm{s}}_{\mathrm{s}} v \cdot \sigma_{\mathrm{abs}}^{v}\left(\nu_{\mathrm{abs}}(t)\right)\right) \cdot t^{\prime}=\nu_{\mathrm{rel}}(t) \cdot t^{\prime}\end{array}$ \\
\hline
\end{tabular}

\section{A.2 Theorem 12}

Theorem 12 (Embedding $\mathbf{A C P}^{\text {dat }} \sqrt{ }$ in $\operatorname{ACP}^{\text {sat }} \mathbf{I} \sqrt{ }$ ) For closed terms, the axioms of $\mathrm{ACP}^{\mathrm{dat}} \sqrt{ }$ are derivable from the axioms of $\mathrm{ACP}^{\mathrm{sat}} \mathrm{I} \sqrt{ }$ and the explicit definitions of the constants and operators $\underline{a}, \sigma_{\mathrm{abs}}, v_{\mathrm{abs}}, \bar{v}_{\mathrm{abs}}$ and $\sqrt{\mathrm{d}}_{\mathrm{d}}$ in Table 25.

\section{Proof.}

To begin with, we show that the axioms of $\mathrm{BPA}^{\text {dat }}$ are derivable for closed terms. Throughout this proof we do not expound the trivial cases.

$$
\begin{aligned}
& \text { DAT7 : } \quad \sigma_{\text {abs }}^{1}(\dot{\delta}) \stackrel{\text { INT } 8}{=} \int_{v \in[0,1)} \sigma_{\text {abs }}^{v}(\tilde{\delta})=\underline{\delta} \\
& \mathrm{A} 6 \mathrm{DAa}: \quad \underline{a}+\underline{\delta}=\int_{v \in[0,1)} \sigma_{\mathrm{abs}}^{v}(\tilde{a})+\int_{v \in[0,1)} \sigma_{\mathrm{abs}}^{v}(\tilde{\delta}) \stackrel{\text { INT11 }}{=} \int_{v \in[0,1)}\left(\sigma_{\mathrm{abs}}^{v}(\tilde{a})+\sigma_{\mathrm{abs}}^{v}(\tilde{\delta})\right) \stackrel{\text { SAT3 }}{=} \\
& \int_{v \in[0,1)} \sigma_{\mathrm{abs}}^{v}(\tilde{a}+\tilde{\delta}) \stackrel{\mathrm{A} 6 \mathrm{SAa}}{=} \int_{v \in[0,1)} \sigma_{\mathrm{abs}}^{v}(\tilde{a})=\underline{a} \\
& \text { DATO2 : } \quad v_{\mathrm{abs}}^{n+1}(\underline{a})=v_{\mathrm{abs}}^{n+1}\left(\int_{v \in[0,1)} \sigma_{\mathrm{abs}}^{v}(\tilde{a})\right) \stackrel{\text { SATO } 6}{=} \int_{v \in[0,1)} v_{\mathrm{abs}}^{n+1}\left(\sigma_{\mathrm{abs}}^{v}(\tilde{a})\right) \stackrel{\text { SATO3 }}{=} \\
& \int_{v \in[0,1)} \sigma_{\mathrm{abs}}^{v}\left(v_{\mathrm{abs}}^{n+1-v}(\tilde{a})\right) \stackrel{\mathrm{SATO} 2}{=} \int_{v \in[0,1)} \sigma_{\mathrm{abs}}^{v}(\tilde{a})=\underline{a} \\
& \text { DAI1 : } \quad \bar{v}_{\mathrm{abs}}^{0}(\underline{a})=\bar{v}_{\mathrm{abs}}^{0}\left(\int_{v \in[0,1)} \sigma_{\mathrm{abs}}^{v}(\tilde{a})\right) \stackrel{\mathrm{SAI} 6}{=} \int_{v \in[0,1)} \bar{v}_{\mathrm{abs}}^{0}\left(\sigma_{\mathrm{abs}}^{v}(\tilde{a})\right) \stackrel{\mathrm{SAI}^{\prime}}{=} \\
& \int_{v \in[0,1)} \sigma_{\mathrm{abs}}^{v}(\widetilde{a})=\underline{a} \\
& \text { DAI2 : } \quad \bar{v}_{\mathrm{abs}}^{n+1}(\underline{a})=\bar{v}_{\mathrm{abs}}^{n+1}\left(\int_{v \in[0,1)} \sigma_{\mathrm{abs}}^{v}(\tilde{a})\right) \stackrel{\mathrm{SAI} 6}{=} \int_{v \in[0,1)} \bar{v}_{\mathrm{abs}}^{n+1}\left(\sigma_{\mathrm{abs}}^{v}(\tilde{a})\right) \stackrel{\text { SAI3 }}{=} \\
& \int_{v \in[0,1)} \sigma_{\text {abs }}^{v}\left(\bar{v}_{\text {abs }}^{n+1-v}\left(\bar{v}_{\mathrm{abs}}^{0}(\tilde{a})\right)\right) \stackrel{\mathrm{SAI} 1}{=} \int_{v \in[0,1)} \sigma_{\text {abs }}^{v}\left(\bar{v}_{\mathrm{abs}}^{n+1-v}(\tilde{a})\right) \stackrel{\text { SAI } 2}{=} \\
& \int_{v \in[0,1)} \sigma_{\mathrm{abs}}^{v}\left(\sigma_{\mathrm{abs}}^{n+1-v}(\dot{\delta})\right) \stackrel{\mathrm{SAT} 2}{=} \int_{v \in[0,1)} \sigma_{\mathrm{abs}}^{n+1}(\dot{\delta}) \stackrel{\text { INT5 }}{=} \sigma_{\mathrm{abs}}^{n+1}(\dot{\delta})
\end{aligned}
$$

Next, we show that the additional axioms for $\mathrm{ACP}^{\text {dat }}$ are derivable for closed terms. 
CF1DA : $\quad \underline{a}\left|\underline{b}=\left(\int_{v \in[0,1)} \sigma_{\mathrm{abs}}^{v}(\tilde{a})\right)\right|\left(\int_{v \in[0,1)} \sigma_{\mathrm{abs}}^{v}(\tilde{b})\right) \stackrel{\text { INT14,15 }}{=}$

$\int_{v \in[0,1)} \int_{w \in[0,1)}\left(\sigma_{\mathrm{abs}}^{v}(\tilde{a}) \mid \sigma_{\mathrm{abs}}^{w}(\tilde{b})\right) \stackrel{\mathrm{INT}^{\mathrm{N} 3,4,11}}{=}$

$\int_{v \in[0,1)} \int_{w \in[0, v)}\left(\sigma_{\mathrm{abs}}^{v}(\tilde{a}) \mid \sigma_{\mathrm{abs}}^{w}(\tilde{b})\right)+\int_{v \in[0,1)}\left(\sigma_{\mathrm{abs}}^{v}(\tilde{a}) \mid \sigma_{\mathrm{abs}}^{v}(\tilde{b})\right)+$

$\int_{v \in[0,1)} \int_{w \in(v, 1)}\left(\sigma_{\text {abs }}^{v}(\tilde{a}) \mid \sigma_{\text {abs }}^{w}(\tilde{b})\right) \stackrel{\text { see I,II,III }}{=}$

$\int_{v \in[0,1)} \sigma_{\mathrm{abs}}^{v}(\dot{\delta})+\int_{v \in[0,1)} \sigma_{\mathrm{abs}}^{v}(\tilde{a} \mid \tilde{b})+\int_{v \in[0,1)} \sigma_{\mathrm{abs}}^{v}(\tilde{\delta}) \stackrel{\text { INT11, SAT3 }}{=}$

$\int_{v \in[0,1)} \sigma_{\mathrm{abs}}^{v}(\dot{\delta}+\tilde{a} \mid \tilde{b}+\tilde{\delta}) \stackrel{\mathrm{A} 6 \mathrm{ID}, \mathrm{A} 6 \mathrm{SAa}}{=} \int_{v \in[0,1)} \sigma_{\mathrm{abs}}^{v}(\tilde{a} \mid \tilde{b}) \stackrel{\mathrm{CF} 1 \mathrm{SA}}{=}$

$\int_{v \in[0,1)} \sigma_{\mathrm{abs}}^{v}(\tilde{c})=\underline{c}$ if $\gamma(a, b)=c$

I. Suppose $p \in[0,1), q \in[0, p)$.

Then $\sigma_{\mathrm{abs}}^{p}(\tilde{a})\left|\sigma_{\mathrm{abs}}^{q}(\tilde{b}) \stackrel{\mathrm{SAU} 1, \mathrm{~A} 6 \mathrm{SAa}}{=} \sigma_{\mathrm{abs}}^{p}(\tilde{a})\right| \sigma_{\mathrm{abs}}^{q}\left(\nu_{\mathrm{abs}}(\tilde{b})+\tilde{\delta}\right) \stackrel{\mathrm{SACM} 4^{\prime}}{=} \sigma_{\mathrm{abs}}^{q}(\tilde{\delta})$.

By INT6, $\int_{w \in[0, p)}\left(\sigma_{\mathrm{abs}}^{p}(\tilde{a}) \mid \sigma_{\mathrm{abs}}^{w}(\tilde{b})\right)=\int_{w \in[0, p)} \sigma_{\mathrm{abs}}^{w}(\tilde{\delta}) \stackrel{\text { INT } 8}{=} \sigma_{\mathrm{abs}}^{p}(\dot{\delta})$.

By INT6, $\int_{v \in[0,1)} \int_{w \in[0, v)}\left(\sigma_{\text {abs }}^{v}(\tilde{a}) \mid \sigma_{\text {abs }}^{w}(\tilde{b})\right)=\int_{v \in[0,1)} \sigma_{\text {abs }}^{v}(\dot{\delta})$.

II. Suppose $p \in[0,1), q=p$.

Then $\sigma_{\mathrm{abs}}^{p}(\tilde{a}) \mid \sigma_{\mathrm{abs}}^{p}(\tilde{b}) \stackrel{\text { SACM5 }}{=} \sigma_{\mathrm{abs}}^{p}(\tilde{a} \mid \tilde{b})$.

By INT6, $\int_{v \in[0,1)}\left(\sigma_{\mathrm{abs}}^{v}(\tilde{a}) \mid \sigma_{\mathrm{abs}}^{v}(\tilde{b})\right)=\int_{v \in[0,1)} \sigma_{\mathrm{abs}}^{v}(\tilde{a} \mid \tilde{b})$.

III. Suppose $p \in[0,1), q \in(p, 1)$.

Then $\sigma_{\mathrm{abs}}^{p}(\tilde{a})\left|\sigma_{\mathrm{abs}}^{q}(\tilde{b}) \stackrel{\mathrm{SAU} 1, \mathrm{~A} 6 \mathrm{SAa}}{=} \sigma_{\mathrm{abs}}^{p}\left(\nu_{\mathrm{abs}}(\tilde{a})+\tilde{\delta}\right)\right| \sigma_{\mathrm{abs}}^{q}(\tilde{b}) \stackrel{\mathrm{SACM} 3^{\prime}}{=} \sigma_{\mathrm{abs}}^{p}(\tilde{\delta})$.

By INT6, $\int_{w \in(p, 1)}\left(\sigma_{\text {abs }}^{p}(\tilde{a}) \mid \sigma_{\text {abs }}^{w}(\tilde{b})\right)=\int_{w \in(p, 1)} \sigma_{\text {abs }}^{p}(\tilde{\delta}) \stackrel{\text { INT5 }}{=} \sigma_{\text {abs }}^{p}(\tilde{\delta})$.

By INT6, $\int_{v \in[0,1)} \int_{w \in(v, 1)}\left(\sigma_{\mathrm{abs}}^{v}(\tilde{a}) \mid \sigma_{\mathrm{abs}}^{w}(\tilde{b})\right)=\int_{v \in[0,1)} \sigma_{\mathrm{abs}}^{v}(\tilde{\delta})$.

CF2DA : The proof is similar to the proof of axiom CF1DA - axiom CF2SA is used instead of axiom CF1SA.

CM2DA : For $p \in[0, \infty), q \in[0,1)$,

$\bar{v}_{\mathrm{abs}}^{p}\left(\sigma_{\mathrm{abs}}^{q}(\tilde{a})\right) \| \bar{v}_{\mathrm{abs}}^{p}(t+\underline{\delta}) \stackrel{\text { see I,IIIIII }}{=} \bar{v}_{\mathrm{abs}}^{p}\left(\sigma_{\mathrm{abs}}^{q}(\tilde{a})\right) \cdot(t+\underline{\delta})$.

By INT6,

$\int_{v \in[0,1)}\left(\bar{v}_{\mathrm{abs}}^{p}\left(\sigma_{\mathrm{abs}}^{v}(\tilde{a})\right) \Downarrow \bar{v}_{\mathrm{abs}}^{p}(t+\underline{\delta})\right)=\int_{v \in[0,1)}\left(\bar{v}_{\mathrm{abs}}^{p}\left(\sigma_{\mathrm{abs}}^{v}(\tilde{a})\right) \cdot(t+\underline{\delta})\right)$.

By SI10, SAI5,6, INT12,13, $\bar{v}_{\text {abs }}^{p}(\underline{a} \Perp(t+\underline{\delta}))=\bar{v}_{\text {abs }}^{p}(\underline{a} \cdot(t+\underline{\delta}))$.

By SIA5, $\underline{a} \Perp(t+\underline{\delta})=\underline{a} \cdot(t+\underline{\delta})$.

I. Suppose $p \in[0, q], q \in[0,1), t \neq \dot{\delta}$.

Then $\bar{v}_{\mathrm{abs}}^{p}\left(\sigma_{\mathrm{abs}}^{q}(\tilde{a})\right)\left\|\bar{v}_{\mathrm{abs}}^{p}(t+\underline{\delta}) \stackrel{\text { Lemma } 9}{=} \bar{v}_{\mathrm{abs}}^{p}\left(\sigma_{\mathrm{abs}}^{q}(\tilde{a})\right)\right\| \sigma_{\mathrm{abs}}^{p}\left(t_{[p]}+\tilde{\delta}\right) \stackrel{\mathrm{SAI}^{\prime}}{=}$

$\sigma_{\text {abs }}^{q}(\tilde{a}) \amalg \sigma_{\text {abs }}^{p}\left(t_{[p]}+\tilde{\delta}\right) \stackrel{\text { SAT2, SACM2 }}{=} \sigma_{\text {abs }}^{p}\left(\sigma_{\text {abs }}^{q-p}(\tilde{a}) \amalg\left(t_{[p]}+\tilde{\delta}\right)\right) \stackrel{\text { Lemma } 1 \text {, SI } 6}{=}$

$\sigma_{\text {abs }}^{p}\left(\sigma_{\text {abs }}^{q-p}(\tilde{a}) \amalg\left(v_{\text {abs }}^{q-p}\left(t_{[p]}+\tilde{\delta}\right)+\bar{v}_{\text {abs }}^{q-p}\left(t_{[p]}+\tilde{\delta}\right)+\sigma_{\text {abs }}^{q-p}(\tilde{\delta})\right)\right) \stackrel{\text { Lemma } 3}{=}$

$\sigma_{\text {abs }}^{p}\left(\sigma_{\text {abs }}^{q-p}(\tilde{a}) \|\left(v_{\text {abs }}^{q-p}\left(t_{[p]}+\tilde{\delta}\right)+\sigma_{\text {abs }}^{q-p}\left(\left(t_{[p]}+\tilde{\delta}\right)_{[q-p]}\right)+\sigma_{\text {abs }}^{q-p}(\tilde{\delta})\right)\right) \stackrel{\text { SAT3, SACM2 }}{=}$

$\sigma_{\text {abs }}^{p}\left(\sigma_{\text {abs }}^{q-p}\left(\tilde{a} \|\left(\left(t_{[p]}+\tilde{\delta}\right)_{[q-p]}+\tilde{\delta}\right)\right)\right) \stackrel{\mathrm{CM} 2 \mathrm{SA}}{=}$

$\sigma_{\mathrm{abs}}^{p}\left(\sigma_{\mathrm{abs}}^{q-p}\left(\tilde{a} \cdot\left(\left(t_{[p]}+\tilde{\delta}\right)_{[q-p]}+\tilde{\delta}\right)\right)\right) \stackrel{\text { SAT1, SAI1, SIA } 6}{=}$

$\sigma_{\text {abs }}^{p}\left(\sigma_{\text {abs }}^{q-p}\left(\tilde{a} \cdot \bar{v}_{\text {abs }}^{0}\left(\left(t_{[p]}+\tilde{\delta}\right)_{[q-p]}+\tilde{\delta}\right)\right)\right) \stackrel{\text { SAT3,5 }}{=}$

$\sigma_{\text {abs }}^{p}\left(\sigma_{\text {abs }}^{q-p}(\tilde{a}) \cdot\left(\sigma_{\text {abs }}^{q-p}\left(\left(t_{[p]}+\tilde{\delta}\right)_{[q-p]}\right)+\sigma_{\text {abs }}^{q-p}(\tilde{\delta})\right)\right)=$

$\sigma_{\mathrm{abs}}^{p}\left(\sigma_{\mathrm{abs}}^{q-p}(\tilde{a}) \cdot\left(\bar{v}_{\mathrm{abs}}^{q-p}\left(t_{[p]}+\tilde{\delta}\right)+\sigma_{\mathrm{abs}}^{q-p}(\tilde{\delta})\right)\right) \stackrel{\text { SI6, SIA } 6}{=}$

$\sigma_{\mathrm{abs}}^{p}\left(\sigma_{\mathrm{abs}}^{q-p}(\tilde{a}) \cdot\left(t_{[p]}+\tilde{\delta}\right)\right) \stackrel{\mathrm{SAT} 5}{=} \sigma_{\mathrm{abs}}^{p}\left(\sigma_{\mathrm{abs}}^{q-p}(\tilde{a})\right) \cdot \sigma_{\mathrm{abs}}^{p}\left(t_{[p]}+\tilde{\delta}\right)=$

$\sigma_{\mathrm{abs}}^{p}\left(\sigma_{\mathrm{abs}}^{q-p}(\tilde{a})\right) \cdot \bar{v}_{\mathrm{abs}}^{p}(t+\underline{\delta}) \stackrel{\mathrm{SIA} 6, \mathrm{SAT} 2, \mathrm{SAI}^{\prime}}{=} \bar{v}_{\mathrm{abs}}^{p}\left(\sigma_{\mathrm{abs}}^{q}(\tilde{a})\right) \cdot(t+\underline{\delta})$

II. Suppose $p \in[0, q], q \in[0,1), t=\dot{\delta}$.

Then $\bar{v}_{\mathrm{abs}}^{p}\left(\sigma_{\mathrm{abs}}^{q}(\tilde{a})\right)\left\|\bar{v}_{\mathrm{abs}}^{p}(t+\underline{\delta}) \stackrel{\mathrm{SAI3}^{\prime}, \text { A6ID }}{=} \sigma_{\mathrm{abs}}^{q}(\tilde{a})\right\| \bar{v}_{\mathrm{abs}}^{p}(\underline{\delta}) \stackrel{\text { Lemma } 11}{=}$

$\sigma_{\mathrm{abs}}^{q}(\tilde{a}) \nVdash \sigma_{\mathrm{abs}}^{1}(\dot{\delta}) \stackrel{\mathrm{SAT} 2, \mathrm{SACM} 2}{=} \sigma_{\mathrm{abs}}^{q}\left(\tilde{a} \| \sigma_{\mathrm{abs}}^{1-q}(\dot{\delta})\right) \stackrel{\mathrm{CM} 2 \mathrm{SA}}{=} \sigma_{\mathrm{abs}}^{q}\left(\tilde{a} \cdot \sigma_{\mathrm{abs}}^{1-q}(\dot{\delta})\right) \stackrel{\mathrm{SAI}^{\prime}, \mathrm{SAT} 2,5}{=}$

$\sigma_{\mathrm{abs}}^{q}(\tilde{a}) \cdot \sigma_{\mathrm{abs}}^{1}(\dot{\delta}) \stackrel{\text { INT8, A6ID }}{=} \sigma_{\mathrm{abs}}^{q}(\tilde{a}) \cdot(t+\underline{\delta}) \stackrel{\mathrm{SAI}^{\prime}}{=} \bar{v}_{\mathrm{abs}}^{p}\left(\sigma_{\mathrm{abs}}^{q}(\tilde{a})\right) \cdot(t+\underline{\delta})$ 
III. Suppose $p \in(q, \infty), q \in[0,1)$.

Then $\bar{v}_{\mathrm{abs}}^{p}\left(\sigma_{\mathrm{abs}}^{q}(\tilde{a})\right)\left\|\bar{v}_{\mathrm{abs}}^{p}(t+\underline{\delta}) \stackrel{\text { Lemma } 9}{=} \bar{v}_{\mathrm{abs}}^{p}\left(\sigma_{\mathrm{abs}}^{q}(\tilde{a})\right)\right\| \sigma_{\mathrm{abs}}^{p}\left(t_{[p]}+\tilde{\delta}\right) \stackrel{\text { SAI1 }, 2,3}{=}$

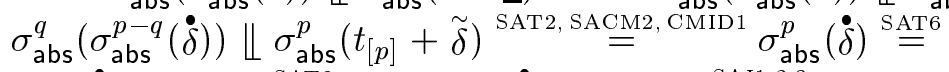

$\sigma_{\mathrm{abs}}^{p}(\dot{\delta}) \cdot(t+\underline{\delta}) \stackrel{\mathrm{SAT} 2}{=} \sigma_{\mathrm{abs}}^{q}\left(\sigma_{\mathrm{abs}}^{p-q}(\dot{\delta})\right) \cdot(t+\underline{\delta}) \stackrel{\mathrm{SAI1,2,3}}{=} \bar{v}_{\mathrm{abs}}^{p}\left(\sigma_{\mathrm{abs}}^{q}(\tilde{a})\right) \cdot(t+\underline{\delta})$

CM3DA : The proof is similar to the proof of axiom CM2DA - axiom CM3SA is used instead of axiom CM2SA.

CM5DA, CM6DA and CM7DA :

The proofs are similar to the proof of axiom CF1DA - axiom SIA6 is used in addition and axioms CM5SA, CM6SA and CM7SA are used instead of axiom CF1SA.

DACM3 : $\quad\left(v_{\text {abs }}^{1}(t)+\underline{\delta}\right)\left|\sigma_{\text {abs }}^{n+1}\left(t^{\prime}\right) \stackrel{\mathrm{SATO} 2,3,6}{=}\left(v_{\mathrm{abs}}^{1}(t)+v_{\mathrm{abs}}^{1}(\underline{\delta})\right)\right| \sigma_{\mathrm{abs}}^{n+1}\left(t^{\prime}\right) \stackrel{\mathrm{SATO} 4}{=}$

$v_{\text {abs }}^{1}(t+\underline{\delta})\left|\sigma_{\text {abs }}^{n+1}\left(t^{\prime}\right) \stackrel{\text { Lemma } 10}{=}\left(\int_{v \in[0,1)} \sigma_{\text {abs }}^{v}\left(\nu_{\text {abs }}\left(t^{\circ}\right)+\tilde{\delta}\right)\right)\right| \sigma_{\text {abs }}^{n+1}\left(t^{\prime}\right) \stackrel{\text { INTs,11,14 }}{=}$

$\int_{v \in[0,1)}\left(\sigma_{\text {abs }}^{v}\left(\nu_{\text {abs }}\left(t^{\circ}\right)+\tilde{\delta}\right) \mid \sigma_{\text {abs }}^{n+1}\left(t^{\prime}\right)\right) \stackrel{\text { SACM3 }}{=} \int_{v \in[0,1)} \sigma_{\text {abs }}^{v}(\tilde{\delta})=\underline{\delta}$

DACM4 : The proof is similar to the proof of axiom DACM3 - axioms INT15 and SACM4 ${ }^{\prime}$ are used instead of axioms INT14 and SACM3'.

D1DA : $\quad \partial_{H}(\underline{a})=\partial_{H}\left(\int_{v \in[0,1)} \sigma_{\mathrm{abs}}^{v}(\tilde{a})\right) \stackrel{\text { INT16, SAD }}{=} \int_{v \in[0,1)} \sigma_{\mathrm{abs}}^{v}\left(\partial_{H}(\tilde{a})\right) \stackrel{\mathrm{D} 1 \mathrm{SA}}{=}$ $\int_{v \in[0,1)} \sigma_{\mathrm{abs}}^{v}(\tilde{a})=\underline{a}$ if $a \notin H$

D2DA : The proof is similar to the proof of axiom D1DA - axiom D2SA is used instead of axioms D1SA.

Finally, we show that the additional axioms for discrete initial abstraction are derivable for closed terms. 
DIA1 : $\quad \sqrt{\mathrm{d}}_{\mathrm{d}} j \cdot G=\sqrt{\mathrm{s}}_{\mathrm{s}} w \cdot G[\lfloor w\rfloor / j] \stackrel{\text { SIA } 1}{=} \sqrt{\mathrm{s}}_{\mathrm{s}} v \cdot G[\lfloor w\rfloor / j][v / w]=\sqrt{\mathrm{s}}_{\mathrm{s}} v \cdot G[\lfloor v\rfloor / j]=$ $\sqrt{\mathrm{s}}_{\mathrm{s}} v \cdot G[i / j][\lfloor v\rfloor / i]=\sqrt{\mathrm{d}} i \cdot G[i / j]$

DIA2 : $\quad \bar{v}_{\mathrm{abs}}^{n}\left(\sqrt{\mathrm{d}}_{\mathrm{d}} i . F\right)=\bar{v}_{\mathrm{abs}}^{n}\left(\sqrt{\mathrm{s}}_{\mathrm{s}} v . F[\lfloor v\rfloor / i]\right) \stackrel{\text { SIA2 }}{=} \bar{v}_{\mathrm{abs}}^{n}(F[\lfloor v\rfloor / i][n / v])=\bar{v}_{\mathrm{abs}}^{n}(F[n / i])$

DIA3 : $\quad \sqrt{\mathrm{d}}_{\mathrm{d}} i \cdot\left(\sqrt{\mathrm{d}}_{\mathrm{d}} j \cdot F\right)=\sqrt{\mathrm{s}}_{\mathrm{s}} v \cdot\left(\sqrt{\mathrm{s}}_{\mathrm{s}} w \cdot F[\lfloor w\rfloor / j]\right)[\lfloor v\rfloor / i]=$ $\sqrt{\mathrm{s}}_{\mathrm{s}} v \cdot\left(\sqrt{\mathrm{s}}_{\mathrm{s}} w \cdot F[\lfloor w\rfloor / j][\lfloor v\rfloor / i]\right) \stackrel{\text { SIA3 }}{=} \sqrt{\mathrm{s}}_{\mathrm{s}} v \cdot F[\lfloor w\rfloor / j][\lfloor v\rfloor / i][v / w]=$ $\sqrt{\mathrm{s}}_{\mathrm{s}} v \cdot F[\lfloor v\rfloor / j][\lfloor v\rfloor / i]=\sqrt{\mathrm{s}}_{\mathrm{s}} v \cdot F[i / j][\lfloor v\rfloor / i]=\sqrt{\mathrm{d}} i . F[i / j]$

DIA4: $\quad G \stackrel{\text { IIA } 4}{=} \sqrt{ }_{\mathrm{s}} v \cdot G=\sqrt{\mathrm{s}}_{\mathrm{s}} v \cdot G[\lfloor v\rfloor / i]=\sqrt{\mathrm{d}}_{\mathrm{d}} i \cdot G$

DIA5 : $\quad$ Suppose $p \in \mathbb{R}_{\geq 0}$ and $\forall n \in \mathbb{N} \bullet \bar{v}_{\text {abs }}^{n}(F)=\bar{v}_{\text {abs }}^{n}\left(F^{\prime}\right)$.

Then $\bar{v}_{\text {abs }}^{p}(F) \stackrel{\text { Lemma } 7}{=} \bar{v}_{\text {abs }}^{p}\left(\sqrt{s}_{\mathrm{s}} v \cdot \bar{v}_{\text {abs }}^{\lfloor v\rfloor}(F)\right) \stackrel{\stackrel{\mathrm{SIA} 2}{=}}{v_{\text {abs }}^{p}}\left(\bar{v}_{\mathrm{abs}}^{\lfloor p\rfloor}(F)\right)=\bar{v}_{\mathrm{abs}}^{p}\left(\bar{v}_{\mathrm{abs}}^{\lfloor p\rfloor}\left(F^{\prime}\right)\right) \stackrel{\text { SIA2} 2}{=}$ $\bar{v}_{\text {abs }}^{p}\left(\sqrt{\mathrm{s}}_{\mathrm{s}} v \cdot \bar{v}_{\mathrm{abs}}^{\lfloor v\rfloor}\left(F^{\prime}\right)\right) \stackrel{\text { Lemma } 7}{=} \bar{v}_{\text {abs }}^{p}\left(F^{\prime}\right)$.

By SIA5, $F=F^{\prime}$.

DIA6 : $\quad \sigma_{\mathrm{abs}}^{n}(\underline{a}) \cdot F=\sigma_{\mathrm{abs}}^{n}\left(\int_{v \in[0,1)} \sigma_{\mathrm{abs}}^{v}(\tilde{a})\right) \cdot F \stackrel{\text { INT10,12, SAT2 }}{=} \int_{v \in[0,1)}\left(\sigma_{\mathrm{abs}}^{n+v}(\tilde{a}) \cdot F\right) \stackrel{\text { Lemma } 7 \text {, SIA6 }}{=}$ $\int_{v \in[0,1)}\left(\sigma_{\text {abs }}^{n+v}(\tilde{a}) \cdot \bar{v}_{\text {abs }}^{n+v}\left(\sqrt{\mathrm{s}}_{\mathrm{s}} w \cdot \bar{v}_{\mathrm{abs}}^{\lfloor w\rfloor}(F)\right)\right) \stackrel{\mathrm{SIA} 2,6}{=}$ $\int_{v \in[0,1)}\left(\sigma_{\mathrm{abs}}^{n+v}(\tilde{a}) \cdot \bar{v}_{\mathrm{abs}}^{n}(F)\right) \stackrel{\text { SAT2, INT10,12 }}{=} \sigma_{\mathrm{abs}}^{n}\left(\int_{v \in[0,1)} \sigma_{\mathrm{abs}}^{v}(\tilde{a})\right) \cdot \bar{v}_{\mathrm{abs}}^{n}(F)=$ $\sigma_{\text {abs }}^{n}(\underline{a}) \cdot \bar{v}_{\text {abs }}^{n}(F)$

DIA7 : $\quad \sigma_{\text {abs }}^{n}\left(\sqrt{d}_{\mathrm{d}} i . F\right)=\sigma_{\mathrm{abs}}^{n}\left(\sqrt{\mathrm{s}}_{\mathrm{s}} v . F[\lfloor v\rfloor / i]\right) \stackrel{\text { SIA7 }}{=} \sigma_{\mathrm{abs}}^{n}(F[\lfloor v\rfloor / i][0 / v])=\sigma_{\mathrm{abs}}^{n}(F[0 / i])$

DIA8 : $\quad\left(\sqrt{\mathrm{d}}_{\mathrm{d}} i \cdot F\right)+G=\left(\sqrt{\mathrm{s}}_{\mathrm{s}} v \cdot F[\lfloor v\rfloor / i]\right)+G \stackrel{\text { Lemma 7 }}{=}$

$\left(\sqrt{s}_{\mathrm{s}} v \cdot F[\lfloor v\rfloor / i]\right)+\left(\sqrt{\mathrm{s}}_{\mathrm{s}} v \cdot \bar{v}_{\mathrm{abs}}^{\lfloor v\rfloor}(G)\right) \stackrel{\mathrm{DISTR}+}{=} \sqrt{\mathrm{s}}_{\mathrm{s}} v \cdot\left(F[\lfloor v\rfloor / i]+\bar{v}_{\mathrm{abs}}^{\lfloor v\rfloor}(G)\right)=$ $\sqrt{\mathrm{s}}_{\mathrm{s}} v \cdot\left(F+\bar{v}_{\mathrm{abs}}^{i}(G)\right)[\lfloor v\rfloor / i]=\sqrt{\mathrm{d}}_{\mathrm{d}} i \cdot\left(F+\bar{v}_{\mathrm{abs}}^{i}(G)\right)$

DIA9 : $\quad\left(\sqrt{\mathrm{d}}_{\mathrm{d}} i \cdot F\right) \cdot G=\left(\sqrt{\mathrm{s}}_{\mathrm{s}} v \cdot F[\lfloor v\rfloor / i]\right) \cdot G \stackrel{\text { SIA9 }}{=} \sqrt{\mathrm{s}}_{\mathrm{s}} v \cdot(F[\lfloor v\rfloor / i] \cdot G)=$ $\sqrt{\mathrm{s}}_{\mathrm{s}} v \cdot(F \cdot G)[\lfloor v\rfloor / i]=\sqrt{\mathrm{d}} i \cdot(F \cdot G)$

DIA10 : $\quad v_{\mathrm{abs}}^{n}\left(\sqrt{\mathrm{d}}_{\mathrm{d}} i . F\right)=v_{\mathrm{abs}}^{n}\left(\sqrt{\mathrm{s}}_{\mathrm{s}} v \cdot F[\lfloor v\rfloor / i]\right) \stackrel{\operatorname{SIA} 10}{=} \sqrt{\mathrm{s}}_{\mathrm{s}} v \cdot v_{\mathrm{abs}}^{n}(F[\lfloor v\rfloor / i])=$ $\sqrt{\mathrm{s}}_{\mathrm{s}} v \cdot v_{\mathrm{abs}}^{n}(F)[\lfloor v\rfloor / i]=\sqrt{\mathrm{d}}_{\mathrm{i}} i \cdot v_{\mathrm{abs}}^{n}(F)$

DIA11, DIA12, DIA13 and DIA14 :

The proofs are similar to the proof of axiom DIA8 - axioms SIA11, SIA12, SIA13 and SIA14 are used instead of axioms SIA8.

DIA15 : The proof is similar to the proof of axiom DIA10 - axiom SIA15 is used instead of axioms SIA10. 